\title{
THE CHARACTERS OF SEMISIMPLE LIE GROUPS
}

\author{
BY \\ HARISH-CHANDRA
}

1. Introduction. Let $\pi$ be a quasi-simple irreducible representation [6(c)] of a connected semisimple Lie group $G$. We denote by $C_{c}^{\infty}(G)$ the space of all complex-valued functions on $G$ which are indefinitely differentiable and which vanish outside a compact set. Then if $d x$ is the Haar measure on $G$, the operator

$$
\pi(f)=\int_{G} f(x) \pi(x) d x \quad\left(f \in C_{c}^{\infty}(G)\right)
$$

is of the trace class and the mapping $T_{\pi}: f \rightarrow s p(\pi(f))$ is a distribution on $G$ which is called the character of $\pi$ (see $[6(d)]$ ). In this paper we shall obtain some results on these characters. Let $l$ be the rank of $G$. We say that an element $x \in G$ is regular if $l$ is exactly the multiplicity of the eigenvalue 1 of the matrix which corresponds to $x$ in the adjoint representation of $G$. The regular elements form an open and dense subset $G^{\prime}$ of $G$. We shall prove (Theorem 6) that $T_{\pi}$ coincides on $G^{\prime}$ (in the sense of distribution theory [11]) with an analytic function $F_{\pi}$ which is given by a formula rather similar to that of Weyl [14(a)] for the compact case (see also [4] and [6(e), p. 511]). However it still remains an open question whether $F_{\pi}$ determines $T_{\pi}$ completely $\left({ }^{1}\right)$.

The central idea of our method is quite simple and can be explained as follows. Let $\mathfrak{g}$ be the complexification of the Lie algebra of $G$ and $\mathfrak{B}$ the universal enveloping algebra of $\mathfrak{g}$. Every element $b \in \mathfrak{B}$ can be regarded as a (left-invariant) differential operator on $G$ and so by duality it operates also on the space of distributions on $G$. Hence the distribution $b T_{\pi}$ is well defined. Let 3 be the center of $\mathfrak{B}$. Then it follows from our assumptions on $\pi$ that $T_{\pi}$ is an "eigen-distribution" of every operator $z \in Z$. Or, in other words, there exists a homomorphism $\chi_{\pi}$ of $B$ into the field of complex numbers such that $z T_{\pi}=\chi_{\pi}(z) T_{\pi}(z \in Z)$. On the other hand, in view of its definition, $T_{\pi}\left(f^{v}\right)$ $=T_{\pi}(f)\left(y \in G, f \in C_{c}^{\infty}(G)\right)$ where $f^{y}$ stands for the function $x \rightarrow f\left(y^{-1} x y\right)(x \in G)$. (We express this property by saying that $T_{x}$ is an invariant distribution.) Now let $x_{0}$ be a regular element in $G$ and $Z_{x_{0}}$ the centralizer of $x_{0}$. Then we can choose a closed normal abelian subgroup $A$ of $Z_{x_{0}}$ such that $x_{0} \in A$ and $Z_{x_{0}} / A$ is finite. Moreover if $B$ is a sufficiently small open neighborhood of $x_{0}$

Received by the editors September 28, 1955.

(1) If $F_{\pi} \neq 0$ one can prove that there is only a finite number of possibilities for $T_{\boldsymbol{\pi}}$ (see [6(h), Theorem 3]). 
in $A, V=\mathrm{U}_{x \in G} x B x^{-1}$ is open in $G . T_{\pi}$ being invariant, it defines in a natural way a distribution $\tau_{\pi}$ on $B$ (regarded as an open submanifold of the Lie group $A)$. Now if we transcribe the differential equations $z T_{\pi}=\chi_{\pi}(z) T_{\pi}(z \in \Re)$ into the corresponding equations for $\tau_{*}$, we find that among the latter there are always some which are of the elliptic type (see for example Gårding [3] for the definition of ellipticity). Hence in view of the recent generaliations (see Schwartz [11, p. 136] and John [8]) of the classical theorem of Bernstein, one can conclude that $\tau_{x}$ is an analytic function on $V$. The precise form of this function can now be obtained by looking more closely at the differential equations for $\tau_{\pi}$.

The contents of this paper are as follows. $\$ 2$ deals with preliminary results. In $\$ 3$ we introduce the notion of a quasi-regular element of $G$. (For us the significance of this concept, which is less strict than that of a regular element-for example in a compact semisimple Lie group, every element is quasi-regular-lies in the fact that the above-mentioned results actually hold on the larger set of quasi-regular elements.) Then, after a brief discussion in $\$ 4$ of some general properties of differential operators on Lie groups, we begin an intensive study of certain special types of operators. The results of this investigation (Theorems 1 and 2) permit us to prove that certain eigendistributions of these operators are actually analytic functions (Theorem 3). The exact form of these functions is obtained in Theorem 4 . These results are then applied to the theory of characters in $\$ 11$. One particular case is considered in greater detail in $\$ 12$ and there we find a rather striking connection (Theorem 8 and Lemma 44) between the characters of the finite- and the infinite-dimensional representations.

A short summary of the results of this paper has been published in [6(h)].

2. Preliminaries. Let $R$ and $C$ denote the fields of real and complex numbers respectively and $(-1)^{1 / 2}$ a fixed square-root of -1 in $C$. Let $g_{0}$ be a semisimple Lie algebra over $R$ and $\mathfrak{g}$ its complexification. We put $B(X, Y)$ $=s p(\operatorname{ad} X$ ad $Y)(X, Y \in g)$ where $X \rightarrow \operatorname{ad} X$ is the adjoint representation of $\mathfrak{g}$. Let $\overline{\mathfrak{u}}$ be a real form of $\mathfrak{g}$. By the conjugation of $\mathfrak{g}$ with respect to $\overline{\mathfrak{u}}$ we mean the autmorphism $\bar{\theta}$ of $g$ over $R$ given by $\bar{\theta}\left(X+(-1)^{1 / 2} Y\right)=X-(-1)^{1 / 2} Y$ $(X, Y \in \overline{\mathfrak{u}})$.We note that if $\mathfrak{h}_{0}$ is a Cartan subalgebra of $\overline{\mathfrak{u}}$, its complexification $\mathfrak{h}$ is a Cartan subalgebra of $\mathfrak{g}$. The real form $\overline{\mathfrak{u}}$ is called compact if the quadratic form $B(X, X)$ is negative definite on $\overline{\mathfrak{u}}$. Let $\eta$ denote the conjugation of $\mathfrak{g}$ with respect to $g_{0}$. The following lemma has been proved by Mostow [10].

Lemma 1 (Mostow). Let $\overline{\mathfrak{h}}_{0}$ be a Cartan subalgebra of $\mathfrak{g}_{0}$ and $\overline{\mathfrak{h}}$ the complexification of $\overline{\mathfrak{h}}_{0}$ in $\mathfrak{g}$. Then there exists a compact real form $\overline{\mathfrak{u}}$ of $\mathfrak{g}$ with the following two properties:

1. $\eta(\overline{\mathfrak{u}})=\overline{\mathfrak{u}}$,

2. $\overline{\mathfrak{h}} \cap \overline{\mathfrak{u}}$ is a Cartan subalgebra of $\overline{\mathfrak{u}}$.

Now let $\mathfrak{u}$ be a fixed compact real form of $\mathfrak{g}$ such that $\eta(\mathfrak{u})=\mathfrak{u}$ and let 
$\mathfrak{f}_{0}=\mathfrak{g}_{0} \cap \mathfrak{u}, \mathfrak{p}_{0}=\mathfrak{g}_{0} \cap(-1)^{1 / 2} \mathfrak{u}$. Then $\mathfrak{g}_{0}=\mathfrak{f}_{0}+\mathfrak{p}_{0}$ and $\mathfrak{g}=\mathfrak{t}+\mathfrak{p}$ where $\mathfrak{l}, \mathfrak{p}$ are the complexifications of $\mathfrak{t}_{0}, \mathfrak{p}_{0}$ respectively in $\mathfrak{g}$ and both the sums are direct. Let $\tilde{\theta}$ denote the conjugation of $\mathfrak{g}$ with respect to $\mathfrak{u}$ and put $\theta=\eta \circ \tilde{\theta}$. Then $\theta$ is an automorphism of $\mathfrak{g}$ over $C$ and $\theta(X+Y)=X-Y(X \in \mathfrak{f}, Y \in \mathfrak{p})$.

Let $G$ be a connected Lie group with the Lie algebra $g_{0}$ and let $x \rightarrow \operatorname{Ad}(x)$ denote the adjoint representation of $G$ on $g$. The following consequence of Lemma 1 was pointed out to me by Chevalley.

Corollary. Let $\overline{\mathfrak{h}}_{0}$ be a Cartan subalgebra of $\mathrm{g}_{0}$. Then we can choose an element $x \in G$ such that if $\mathfrak{h}_{0}=\operatorname{Ad}(x) \overline{\mathfrak{h}}_{0}$,

$$
\mathfrak{h}_{0}=\mathfrak{h}_{0} \cap \mathfrak{l}_{0}+\mathfrak{h}_{0} \cap \mathfrak{p}_{0} .
$$

Choose $\overline{\mathfrak{u}}$ in accordance with Lemma 1 and let $\bar{\theta}$ denote the conjugation of $\mathfrak{g}$ with respect to $\overline{\mathfrak{u}}$. Put $\overline{\mathfrak{f}}_{0}=\overline{\mathfrak{u}} \cap \mathfrak{g}_{0}, \overline{\mathfrak{p}}_{0}=\left((-1)^{1 / 2} \overline{\mathfrak{u}}\right) \cap \mathfrak{g}_{0}$. Then $\mathfrak{g}_{0}$ is the direct sum of $\overline{\mathfrak{t}}_{0}$ and $\mathfrak{p}_{0}$ and it follows from a theorem of Cartan $[1(\mathrm{~b})]$ that $\overline{\mathfrak{f}}_{0}$ $=\operatorname{Ad}\left(x^{-1}\right) \mathfrak{l}_{0}$ for some $x \in K$. Now suppose $X \in \overline{\mathfrak{t}}_{0}, Y \in \mathfrak{p}_{0}$. Then $B(X, Y)$ $=B(\bar{\theta}(X), \bar{\theta}(Y))=B(X,-Y)$ and therefore $X$ and $Y$ are orthogonal under the bilinear form $B$. But $B(X, X)<0$ if $X \in \mathfrak{l}_{0}(X \neq 0)$ and so it follows that $\mathfrak{p}_{0}$ is exactly the set of elements of $\mathfrak{g}_{0}$ orthogonal to $\overline{\mathfrak{f}}_{0}$ under $B$. A similar relationship holds between $\mathfrak{f}_{0}$ and $\mathfrak{p}_{0}$ and therefore also between $\overline{\mathfrak{t}}_{0}=\operatorname{Ad}\left(x^{-1}\right) \mathfrak{f}_{0}$ and $\operatorname{Ad}\left(x^{-1}\right) \mathfrak{p}_{0}$. This proves that $\operatorname{Ad}\left(x^{-1}\right) \mathfrak{p}_{0}=\mathfrak{p}_{0}$. Now let $\mathfrak{h}_{0}=\operatorname{Ad}(x) \overline{\mathfrak{h}}_{0}$. If $\overline{\mathfrak{h}}$ is the complexification of $\overline{\mathfrak{h}}_{0}$ in $\mathfrak{g}$, it follows from the second condition of Lemma 1 that $\overline{\mathfrak{h}}$ is also the complexification of $\overline{\mathfrak{h}} \cap \overline{\mathfrak{u}}$ and therefore $\bar{\theta}(\overline{\mathfrak{h}})=\overline{\mathfrak{h}}$. But obviously $\bar{\theta}\left(g_{0}\right)=g_{0}$ and therefore

$$
\bar{\theta}\left(\overline{\mathfrak{h}}_{0}\right)=\bar{\theta}\left(\overline{\mathfrak{h}} \cap \mathfrak{g}_{0}\right)=\overline{\mathfrak{h}} \cap \mathfrak{g}_{0}=\overline{\mathfrak{h}}_{0} .
$$

This shows that $\overline{\mathfrak{h}}_{0}=\overline{\mathfrak{h}}_{0} \cap \overline{\mathfrak{f}}_{0}+\overline{\mathfrak{h}}_{0} \cap \mathfrak{p}_{0}$ and hence

$$
\mathfrak{h}_{0}=\mathfrak{h}_{0} \cap \mathfrak{t}_{0}+\mathfrak{h}_{0} \cap \mathfrak{p}_{0} .
$$

Let $\mathfrak{a}_{0}$ be a maximal abelian subspace of $\mathfrak{p}_{0}$. For each (real-valued) linear function $\lambda$ on $a_{0}$ let $g_{0, \lambda}$ be the set of all $X \in \mathfrak{g}_{0}$ such that $[H, X]=\lambda(H) X$ for all $H \in \mathfrak{a}_{0}$. Let $\Sigma$ be the set of those $\lambda \neq 0$ for which $\mathfrak{g}_{0, \lambda} \neq 0$. Then $\Sigma$ is a finite set. Let $K$ denote the analytic subgroup of $G$ corresponding to $\mathfrak{f}_{0}$.

Lemma $\left({ }^{2}\right)$ 2. Let $\mathfrak{h}_{1}, \mathfrak{h}_{2}$ be two Cartan subalgebras of $\mathfrak{g}_{0}$ both invariant under $\theta$ and such that $\mathfrak{h}_{i} \cap \mathfrak{p}_{0} \subset \mathfrak{a}_{0}(i=1,2)$. Let $\Sigma_{i}$ be the set of those $\alpha \in \Sigma$ which vanish identically on $\mathfrak{h}_{i} \cap \mathfrak{p}_{0}(i=1,2)$. Then if $\Sigma_{1}=\Sigma_{2}$ there exists an element $k \in K$ such that $\mathfrak{h}_{2}=\operatorname{Ad}(k) \mathfrak{h}_{1}$.

Choose an element $H_{0} \in \mathfrak{h}_{1} \cap \mathfrak{p}_{0}$ such that $\alpha\left(H_{0}\right) \neq 0(\alpha \in \Sigma)$ unless $\alpha \in \Sigma_{1}$. Also let $\mathfrak{a}_{1}$ denote the set of all those $H \in \mathfrak{a}_{0}$ for which $\alpha(H)=0$ for every $\alpha \in \Sigma_{1}$. Obviously $\mathfrak{a}_{1} \supset \mathfrak{h}_{1} \cap \mathfrak{p}_{0}$. We shall now show that $\mathfrak{a}_{1}=\mathfrak{h}_{1} \cap \mathfrak{p}_{0}$. Let $\mathfrak{q}_{0}$ be

(2) This result had also been obtained independently by A. Borel. 
the centralizer of $H_{0}$ in $\mathfrak{t}_{0}$. Then it is obvious that

$$
\mathfrak{q}_{0} \subset \mathfrak{g}_{0,0}+\sum_{\alpha \in \Sigma_{1}} \mathfrak{g}_{0, \alpha}
$$

and therefore $\left[\mathfrak{q}_{0}, \mathfrak{a}_{1}\right]=\{0\}$. But it is clear that $\mathfrak{h}_{1} \cap \mathfrak{f}_{0} \subset \mathfrak{q}_{0}$ and so $\mathfrak{a}_{1}+\mathfrak{h}_{1} \cap \mathfrak{f}_{0}$ is an abelian algebra. However

$$
\mathfrak{a}_{1}+\mathfrak{h}_{1} \cap \mathfrak{f}_{0} \supset \mathfrak{h}_{1} \cap \mathfrak{p}_{0}+\mathfrak{h}_{1} \cap \mathfrak{f}_{0}=\mathfrak{h}_{1} .
$$

Therefore since $\mathfrak{h}_{1}$ is maximal abelian in $\mathfrak{g}_{0}, \mathfrak{a}_{1}=\mathfrak{h}_{1} \cap \mathfrak{p}_{0}$.

This proves that $\mathfrak{h}_{1} \cap \mathfrak{p}_{0}$ is completely determined by $\Sigma_{1}$. Hence if $\Sigma_{1}=\Sigma_{2}$, $\mathfrak{h}_{1} \cap \mathfrak{p}_{0}=\mathfrak{h}_{2} \cap \mathfrak{p}_{0}$. Then $\mathfrak{q}_{0}$, as defined above, is the centralizer in $\mathfrak{t}_{0}$ of $\mathfrak{h}_{1} \cap \mathfrak{p}_{0}$ $=\mathfrak{h}_{2} \cap \mathfrak{p}_{0}$. Replacing $G$, if necessary, by its adjoint group we may assume that $K$ is compact (see Mostow [10]). Then if $Q$ is the centralizer $\left.{ }^{8}\right)$ of $\mathfrak{h}_{1} \cap p_{0}$ in $K, Q$ being a closed subgroup of $K$, is compact. Also its Lie algebra is $q_{0}$. Since it is obvious that both $\mathfrak{h}_{1} \cap \mathfrak{f}_{0}$ and $\mathfrak{h}_{2} \cap \mathfrak{f}_{0}$ are maximal abelian subalgebras of $\mathfrak{q}_{0}$ it follows, in view of the compactness of $Q$, that $\mathfrak{h}_{2} \cap \mathfrak{f}_{0}=\operatorname{Ad}(k)\left(\mathfrak{h}_{1} \cap \mathfrak{l}_{0}\right)$ for some $k \in Q$. But then

$$
\begin{aligned}
\operatorname{Ad}(k) \mathfrak{h}_{1} & =\operatorname{Ad}(k)\left(\mathfrak{h}_{1} \cap \mathfrak{l}_{0}\right)+\operatorname{Ad}(k)\left(\mathfrak{h}_{1} \cap \mathfrak{p}_{0}\right) \\
& =\mathfrak{h}_{2} \cap \mathfrak{l}_{0}+\mathfrak{h}_{1} \cap \mathfrak{p}_{0}=\mathfrak{h}_{2} \cap \mathfrak{l}_{0}+\mathfrak{h}_{2} \cap \mathfrak{p}_{0}=\mathfrak{h}_{2} .
\end{aligned}
$$

We say that two Cartan subalgebras $\mathfrak{h}_{1}, \mathfrak{h}_{2}$ are conjugate (or lie in the same conjugacy class) if $\mathfrak{h}_{2}=\operatorname{Ad}(x) \mathfrak{h}_{1}$ for some $x \in G$.

COROLLARY. We can select a finite number of Cartan subalgebras $\mathfrak{h}_{1}, \cdots, \mathfrak{h}_{r}$ of $\mathfrak{g}_{0}$ with the following two properties. $\theta\left(\mathfrak{h}_{i}\right)=\mathfrak{h}_{i}(i=1, \cdots, r)$ and if $\mathfrak{h}_{0}$ is any Cartan subalgebra of $\mathfrak{g}_{0}, \mathfrak{h}_{0}$ is conjugate to $\mathfrak{h}_{i}$ for exactly one $i$.

The set $\Sigma$ being finite, there is only a finite number of possibilities for the subsets $\Sigma_{1}, \Sigma_{2}$ of Lemma 2 . Hence if we combine the above results with the corollary to Lemma 1, our assertion follows from the fact (see Cartan [1(a) ]) that any two maximal abelian subspaces of $p_{0}$ are conjugate under $K$.

Let $\lambda$ be an indeterminate. For any $x \in G$ consider the polynomial

$$
D(x, \lambda)=\operatorname{det}\{(\lambda+1) I-\operatorname{Ad}(x)\}
$$

in $\lambda$. (Here $I$ is the identity mapping of g.) Let $\lambda^{l}$ be the highest power of $\lambda$ which divides $D(x, \lambda)$ for every $x$. Then $l$ is called the rank of $G$ (or of $g_{0}$ ) and, as is well known, every Cartan subalgebra of $g_{0}$ has (real) dimension $l$. Now clearly if $n=\operatorname{dim} \mathfrak{g}$,

$$
D(x, \lambda)=\lambda^{n}+\sum_{r=l}^{n-1}(-1)^{n-r} D_{r}(x) \lambda^{r}
$$

( $\left.{ }^{3}\right)$ Let $x$ and $X$ be two elements in $G$ and $g$ respectively. It is convenient to say that $x$ and $X$ commute if $\operatorname{Ad}(x) X=X$. 
where $D_{r}(l \leqq r<n)$ are analytic functions on $G$ and $D_{l}$ is not identically zero. Let $S$ denote the set of zeros of $D_{l}$ on $G$. We call $S$ the singular set of $G$. Obviously it is a closed nowhere dense set. A point $x \in G$ is called singular or regular according as it lies in $S$ or not. If $z$ lies in the center $Z$ of $G, \operatorname{Ad}(z x)$ $=\operatorname{Ad}(x) \quad(x \in G)$ and therefore $Z S=S$. Similarly $D\left(y x y^{-1}, \lambda\right)=D(x, \lambda)$ $(x, y \in G)$ and therefore $y S y^{-1}=S$.

An element $X \in \mathfrak{g}$ is called regular if the multiplicity of the eigenvalue zero of ad $X$ is exactly $l$. It is well known (see Weyl [14(b)]) that if $X$ is regular, its centralizer in $\mathfrak{g}$ is a Cartan subalgebra of $\mathfrak{g}$ and conversely every Cartan subalgebra contains a regular element. Let $A$ be a maximal abelian subgroup of $G$. We say that $A$ is a Cartan subgroup $\left({ }^{4}\right)$ of $\mathfrak{g}$ if its connected component of 1 is not contained in $S$. Obviously a Cartan subgroup is closed and hence it is a Lie subgroup of $G$.

Lemma 3. The Lie algebra of any Cartan subgroup of $G$ is a Cartan subalgebra of $\mathfrak{g}_{0}$. Conversely if $\mathfrak{h}_{0}$ is any Cartan subalgebra of $\mathfrak{g}_{0}$, there is exactly one Cartan subgroup $A$ of $G$ whose Lie algebra is $\mathfrak{h}_{0}$. If $H$ is a regular element in $\mathfrak{h}_{0}$, $A$ coincides with the centralizer $\left(^{3}\right)$ of $H$ in $G$.

Corollary. Let $Z_{x}$ denote the centralizer of a regular element $x$ in $G$. Then $Z_{x}$ contains exactly one Cartan subgroup $A$ of $G$. Moreover $A$ is normal in $Z_{x}$ and $Z_{x} / A$ is finite.

Before proving the above lemma and its corollary, we need a few auxiliary results. Let $G_{c}$ be a complex analytic group with the Lie algebra $\mathfrak{g}$ and let $S_{c}$ denote the singular set of $G_{c}$. Then if $\mathfrak{h}$ is a Cartan subalgebra of $\mathfrak{g}$, we consider the complex analytic subgroup $A_{c}$ corresponding to $\mathfrak{h}$. Let $G_{c}^{\prime}$ denote the complement of $S_{c}$ in $G_{c}$.

Lemma 4. $G_{c}^{\prime}$ is connected and $G_{c}^{\prime} \subset \bigcup_{z \in G_{c}} z A_{c} z^{-1}$. Moreover if $H_{0}$ is a regular element in $\mathfrak{h}$, the centralizer of $H_{0}$ in $G_{c}$ coincides with $A_{c}$.

Let $z \rightarrow \operatorname{Ad}(z)\left(z \in G_{c}\right)$ denote the adjoint representation of $G_{c}$ on $\mathfrak{g}$. Then it is a complex representation (see $[6(\mathrm{~g}), \S 6]) . \lambda$ being an indeterminate, consider the polynomial

$$
D(z, \lambda)=\operatorname{det}\{(\lambda+1) I-\operatorname{Ad}(z)\}
$$

where $I$ is the identity mapping of $g$. The coefficients of this polynomial are holomorphic functions on $G$. $l$ being the rank of $\mathfrak{g}$, let $(-1)^{l} D_{l}(z)$ denote the coefficient of $\lambda^{l}$. Then $S_{c}$ is the set of zeros of the holomorphic function $D_{l}$. Since $D_{l}$ is not identically zero, $S_{c}$ is a complex-analytic subvariety of $G_{c}$ of one complex dimension (and therefore two real dimensions) less. Hence $G_{c}^{\prime}$ is connected.

Put $V^{\prime}=\bigcup_{z \in G_{c}} z A_{c}^{\prime} z^{-1}$ where $A_{c}^{\prime}=A_{c} \cap G_{c}^{\prime}$. It is obvious that $V \subset G_{c}^{\prime}$.

(4) The definition of a Cartan subgroup given in $[6(\mathrm{~h})]$ is not quite correct. 
Moreover if $a$ is an element in $A_{c}$ sufficiently near 1 but different from 1, it is easily seen that $a$ is regular and therefore $V^{\prime}$ is not empty. Therefore since $G_{c}^{\prime}$ is connected, in order to prove the second statement of the lemma, it would be sufficient to show that $V^{\prime}$ is both open and closed in $G_{c}^{\prime}$. Let $G_{c}^{*}$ be the factor space $G_{c} / A_{c}$ consisting of all cosets of the form $z A_{c}\left(z \in G_{c}\right)$. Put $a^{z *}=z a z^{-1}\left(z \in G_{c}, a \in A_{c}\right)$ where $z \rightarrow z^{*}$ is the natural mapping of $G_{c}$ on $G_{c}^{*}$. Then an easy computation shows that the holomorphic mapping $\phi:\left(z^{*}, a\right)$ $\rightarrow a^{2 *}\left(z^{*} \in G_{c}^{*}, a \in A_{c}\right)$ of $G_{c}^{*} \times A_{c}$ into $G_{c}$ is everywhere regular on $G_{c} \times A_{c}^{\prime}$ and therefore we conclude that $V^{\prime}=\phi\left(G_{c}^{*} \times A_{c}^{\prime}\right)$ is open in $G_{c}$. So now it remains to show that $V^{\prime}$ is closed in $G_{c}^{\prime}$. Introduce some lexicographic order among the roots of $\mathfrak{g}$ (with respect to $\mathfrak{h}$ ) and for each root $\alpha$ select an element $X_{\alpha} \neq 0$ such that $\left[H, X_{\alpha}\right]=\alpha(H) X_{\alpha}$ for every $H \in \mathfrak{h}$. Then $\mathfrak{n}=\sum_{\alpha>0} C X_{\alpha}$ is a nilpotent subalgebra of $\mathfrak{g}$. Let $\mathfrak{h}_{0}$ be the real subspace of $\mathfrak{h}$ consisting of those elements where every root $\alpha$ takes a pure imaginary value. It is possible to choose (see Weyl [14(b)]) a compact real form $\overline{\mathfrak{u}}$ of $\mathfrak{g}$ which contains $\mathfrak{h}_{0}$. Let $U$ and $N$ be the (real) analytic subgroups of $G_{c}$ corresponding to $\overline{\mathfrak{u}}$ and $\mathfrak{n}$ respectively. Then $U$ is compact, $G_{c}=U A_{c} N$ and $A_{c} N$ is closed (see Iwasawa [7]). Hence

$$
V=\bigcup_{u \in U} u A_{c} N u^{-1}
$$

is also a closed set. Moreover it is obvious that

$$
V \cap G_{c}^{\prime}=\bigcup_{u \in U} u\left(A_{c} N\right)^{\prime} u^{-1}
$$

where $\left(A_{c} N\right)^{\prime}=A_{c} N \cap G_{c}^{\prime}$. Also we know (Lemmas 8 and 10 of [6(e)]) that

$$
\left(A_{c} N\right)^{\prime}=\bigcup_{n \in N} n A_{c}^{\prime} n^{-1}
$$

and therefore $V \cap G_{c}^{\prime}=V^{\prime}$. But since $G_{c}=U A_{c} N=U N A_{c}$, it follows that $V^{\prime} \subset V$ and so $V^{\prime}=V \cap G_{c}^{\prime}$. This proves that $V^{\prime}$ is closed in $G_{c}^{\prime}$.

Now let $H_{0}$ be a regular element in $\mathfrak{h}$ and suppose $\operatorname{Ad}(x) H_{0}=H_{0}(x \in G)$. Then $x=u n a\left(u \in U, n \in N, a \in A_{c}\right)$ and so $\operatorname{Ad}(n) H_{0}=\operatorname{Ad}\left(u^{-1}\right) H_{0}$. Since $\mathfrak{h}$ is the centralizer of $H_{0}$ in $\mathfrak{g}$, we conclude that $\operatorname{Ad}(n) \mathfrak{h}=\operatorname{Ad}\left(u^{-1}\right) \mathfrak{h}$. But $\operatorname{Ad}(n) \mathfrak{h}$ $\subset \mathfrak{h}+\mathfrak{n}$ and $\operatorname{Ad}\left(u^{-1}\right) \mathfrak{h}_{0} \subset \overrightarrow{\mathfrak{u}}$. Moreover if $(\mathfrak{h}+\mathfrak{n}) \cap \overline{\mathfrak{u}}=\mathfrak{h}_{1}$, it is obvious that $\left[\mathfrak{h}_{0}, \mathfrak{h}_{1}\right] \subset \mathfrak{n} \cap \overline{\mathfrak{u}}=\{0\}$. Therefore since $\mathfrak{h}_{0}$ is maximal abelian in $\overline{\mathfrak{u}}$ it follows that $\mathfrak{h}_{1}=\mathfrak{h}_{0}$. This shows that $\operatorname{Ad}\left(u^{-1}\right) \mathfrak{h}_{0}=\mathfrak{h}_{0}$ and so $\operatorname{Ad}(n) \mathfrak{h}=\operatorname{Ad}\left(u^{-1}\right) \mathfrak{h}=\mathfrak{h}$. But from Lemma 8 of [6(e)], this implies that $n=1$ and hence Ad $\left(u^{-1}\right) H_{0}$ $=H_{0}$. Let $H_{1}, H_{2}$ be elements in $\mathfrak{h}_{0}$ such that $H_{0}=H_{1}+(-1)^{1 / 2} H_{2}$. Then it is obvious that $\operatorname{Ad}\left(u^{-1}\right) H_{i}=H_{i}, i=1,2$. Moreover since $H_{0}$ is regular, no root of $\mathfrak{g}$ (with respect to $\mathfrak{h}$ ) can vanish at both $H_{1}$ and $H_{2}$. Therefore we can choose $t \in R$ such that $H=H_{1}+t H_{2}$ is a regular element in $\mathfrak{h}_{0}$. Let $A_{0}$ be the analytic subgroup of $U$ corresponding to $\mathfrak{h}_{0}$. Then since $U$ is compact and $\operatorname{Ad}(u) H=H$, it follows (see Stiefel [12, Satz 7]) that $u \in A_{0}$. This shows that $x \in A_{c}$ and so the lemma is now proved completely. 
CoRollary. Let $A_{x}$ denote the centralizer (in $G_{c}$ ) of a regular element $x$ in $G_{c}$. Then $A_{x}$ has only a finite number of connected components and its Lie algebra is a Cartan subalgebra of $\mathfrak{g}$.

From Lemma 4 we can choose $z \in G_{c}$ such that $a=z^{-1} x z \in A_{c}$. It is obvious that $a$ is regular and therefore $\mathfrak{h}$ is the centralizer $\left(^{3}\right)$ of $a$ in $\mathfrak{g}$. Let $A^{*}$ be the normalizer of $A_{c}$ in $G_{c}$. It is clear that $z A_{c} z^{-1}$ is the connected component of 1 in $A_{x}$ and $A_{x} \subset z A^{*} z^{-1}$. Hence in order to prove the corollary it is sufficient to show that $A^{*} / A_{c}$ is finite. Let $y \in A^{*}$. Then $y=u n b\left(u \in U, n \in N, b \in A_{c}\right)$ and $\operatorname{Ad}(n) \mathfrak{h}=\operatorname{Ad}\left(u^{-1}\right) \mathfrak{h}$. By the argument which we have already used in the proof of Lemma 4, it follows that $n=1$ and so $u \in A^{*} \cap U$. This proves that $A^{*} / A_{c} \simeq\left(A^{*} \cap U\right) /\left(A_{c} \cap U\right)$. But since $\mathfrak{h}$ is its own normalizer in $\mathfrak{g}$, we conclude that $A^{*} / A_{c}$ is both discrete and compact. Therefore it is finite.

Now we come to Lemma 3 and its corollary. If $Z$ is the center of $G$, it is obviously permissible for our proof to replace $G$ by $G / Z$. Hence we may assume that there exists a complex analytic group $G_{c}$ with the Lie algebra $g$ such that $G$ is the (real) analytic subgroup of $G_{c}$ corresponding to $g_{0}$. Then if $S_{c}$ and $G_{c}^{\prime}$ are defined as above, it is obvious that $G \cap G_{c}^{\prime}=G^{\prime}$ and $G \cap S_{c}=S$. Let $A$ be a Cartan subgroup of $G$. We denote by $A_{0}$ the connected component of 1 and by $\mathfrak{h}_{0}$ the Lie algebra of $A$. Choose a regular element $a \in A_{0}$. $A$ being abelian, $a=\exp H$ for some $H \in \mathfrak{h}_{0}$ and it is obvious that $H$ is regular. Let $A_{c}$ and $\mathfrak{h}$ be the centralizers of $H$ in $G_{c}$ and $\mathfrak{g}$ respectively. From Lemma 4 , $A_{c}$ is abelian and hence it is clear that $A=A_{c} \cap G$. Therefore $\mathfrak{h}_{0}=\mathfrak{h} \cap \mathfrak{g}_{0}$ and this shows that $\mathfrak{h}_{0}$ is a Cartan subalgebra of $g_{0}$ and $A$ is the centralizer in $G$ of any regular element in $\mathfrak{h}_{0}$. Conversely let $\mathfrak{h}_{0}$ be a given Cartan subalgebra of $\mathfrak{g}_{0}$ and $H$ a regular element in $\mathfrak{h}_{0}$. Then if $A_{c}$ is the centralizer of $H$ in $G_{c}$, we know from Lemma 4 that $A_{c}$ is abelian. Hence it is obvious that $A=A_{c} \cap G$ is a maximal abelian subgroup of $G$. Moreover if $t$ is sufficiently small and positive $\exp t H$ is evidently regular. Therefore $A$ is a Cartan subgroup of $G$. All the statements of Lemma 3 are now obvious. However we still have to prove the corollary. $x$ being a regular element in $G$, let $\mathfrak{h}_{x}$ denote the centralizer $\left.{ }^{3}\right)$ of $x$ in $\mathfrak{g}$ and $A_{c}$ the analytic subgroup of $G_{c}$ corresponding to $\mathfrak{h}_{x}$. Then if $A_{x}$ is the centralizer of $x$ in $G_{c}$, we have seen (corollary to Lemma 4) that $A_{c}$ is normal in $A_{x}$ and $A_{x} / A_{c}$ is finite. Therefore $\left(A_{x} \cap G\right) /\left(A_{c} \cap G\right)$ is also finite. But it is obvious that $Z_{x}=A_{x} \cap G$ and, as we saw above, $A=A_{c} \cap G$ is the Cartan subgroup of $G$ corresponding to $\mathfrak{h}_{x} \cap \mathfrak{g}_{0}$. Moreover since $\mathfrak{h}_{x} \cap \mathfrak{g}_{0}$ is the Lie algebra of $Z_{x}$, it is clear that $A$ is the only Cartan subgroup of $G$ contained in $Z_{x}$.

Lemma 5. Let $\mathfrak{h}_{1}, \cdots, \mathfrak{h}_{r}$ be defined as in the corollary to Lemma 2 and let $A_{i}$ be the Cartan subgroup of $G$ which corresponds to $\mathfrak{h}_{i}(i=1, \cdots, r)$. Then

$$
G^{\prime} \subset \bigcup_{i=1}^{r} \underset{x \in G}{\bigcup} x A_{i} x^{-1}
$$


Since $A_{i} \supset Z$ for every $i$, it is again permissible to replace $G$ by $G / Z$. Therefore, as in the proof of Lemma 3, we may assume that $G \subset G_{c}$. Let $y$ be a regular element in $G$ and let $\mathfrak{h}_{\nu}$ denote the centralizer of $y$ in $\mathfrak{g}$. Then as we have seen above $\mathfrak{h}_{y} \cap \mathfrak{g}_{0}$ is a Cartan subalgebra of $\mathfrak{g}_{0}$ and therefore $\mathfrak{h}_{y} \cap \mathfrak{g}_{0}$ $=\operatorname{Ad}(x) \mathfrak{h}_{i}$ for some $x \in G$ and some $i(1 \leqq i \leqq r)$. Now if $A_{y}$ is the Cartan subgroup of $G$ corresponding to $\mathfrak{h}_{y} \cap \mathfrak{g}_{0}$, it follows from Lemma 3 that $A_{y}=x A_{i} x^{-1}$. But since $A_{y}$ is the centralizer of $\mathfrak{h}_{y}$ in $G$ (see Lemma 3), $y \in x A_{i} x^{-1}$.

Two Cartan subgroups $A$ and $B$ of $G$ are said to be conjugate (or to lie in the same conjugacy class) if $B=x A x^{-1}$ for some $x \in G$.

Lemma 6. Every Cartan subgroup $A$ of $G$ is conjugate to $A_{i}$ for exactly one $i$ $(1 \leqq i \leqq r)$.

This follows immediately from Lemma 3 and the corollary to Lemma 2.

Let $\mathfrak{h}_{0}$ be a Cartan subalgebra of $\mathfrak{g}_{0}$ such that $\theta\left(\mathfrak{h}_{0}\right)=\mathfrak{h}_{0}$. Let $A$ be the centralizer of $\mathfrak{h}_{0}$ in $G$ and $\bar{M}$ the connected component of 1 in the centralizer of $\mathfrak{h}_{0} \cap \mathfrak{p}_{0}$ in $K$. We put $M=\bar{M} A$. Let $\mathfrak{m}_{\mathfrak{t}_{0}}$ be the centralizer of $\mathfrak{h}_{0} \cap \mathfrak{p}_{0}$ in $\mathfrak{t}_{0}$. Then $\mathfrak{m}_{\mathfrak{t}_{0}}$ is the Lie algebra of $\bar{M}$ and it is obvious that $\operatorname{Ad}(a) \mathfrak{m}_{\mathfrak{t}_{0}}=\mathfrak{m}_{\mathfrak{t}_{0}}$ for $a \in A$. Hence $M$ is a group. Let $\bar{B}$ denote the centralizer of $\mathfrak{m}_{\mathfrak{t}_{0}}$ in $A \cap K$.

Lemma 7. Let $A_{+}$denote the analytic subgroup of $G$ corresponding to $\mathfrak{h}_{0} \cap \mathfrak{p}_{0}$. Then $A=(A \cap K) A_{+}, M \cap K=\bar{M} \bar{B}$ and $\bar{B}$ is the center of $M \cap K$. Moreover

$$
M \cap K=\bigcup_{m \in \bar{M}} m(A \cap K) m^{-1}
$$

and $A \cap K=\bar{A} \bar{B}$ where $\bar{A}$ is the connected component of 1 in $A \cap K$. Finally $M=\bar{M} \bar{B} A_{+}$and $\bar{B} A_{+}$is the center of $M$.

Let $\exp \mathfrak{p}_{0}$ denote the set of all elements in $G$ of the form $\exp X\left(X \in p_{0}\right)$. Then we can "extend" $\theta$ to an automorphism of $G$ such that $\theta(k p)=k p^{-1}$ where $k \in K$ and $p \in \exp \mathfrak{p}_{0}$. Since $\theta\left(\mathfrak{h}_{0}\right)=\mathfrak{h}_{0}$, it follows that $\theta(A)=A$ and therefore if $a \in A$ and $a=k p\left(k \in K, p \in \exp p_{0}\right), p^{2}=\theta\left(a^{-1}\right) a$ is also in $A$. But with regard to the positive definite quadratic form $-B(\theta(Y), Y)\left(Y \in g_{0}\right)$ on $g_{0}$, ad $X\left(X \in p_{0}\right)$ is a symmetric operator and hence $\exp (\operatorname{ad} X) Y \neq Y\left(Y \in g_{0}\right)$ unless $[X, Y]=0$. Therefore if $p=\exp X\left(X \in p_{0}\right)$ we can conclude that $($ ad $X) \mathfrak{h}_{0}=\{0\}$ and $X \in \mathfrak{h}_{0} \cap \mathfrak{p}_{0}$. This proves that $p \in A_{+}$and so $k \in A \cap K$. Now since $Z \subset \bar{B} \subset A \cap K$, it is clearly permissible to replace $G$ by $G / Z$ in proving the rest of the above lemma. So we may assume that $K$ and therefore also $A \cap K$ and $\bar{M}$ are compact. It is obvious that $\mathfrak{h}_{0} \cap \mathfrak{l}_{0}$ is a maximal abelian subalgebra of $\mathfrak{m}_{\mathfrak{t}_{0}}$. Hence $\bar{M}$ being compact, it follows (see Weyl [14(b)]) that

$$
\bar{M}=\bigcup_{m \in \bar{M}} m \bar{A} m^{-1}
$$

and so $\bar{A}$ contains the center of $\bar{M}$. Now complexify $\mathfrak{h}_{0}, \mathfrak{m}_{\mathfrak{t}}$ to $\mathfrak{h}$, $\mathfrak{m}_{\mathfrak{t}}$ respectively and for every root $\alpha$ (of $\mathfrak{g}$ with respect to $\mathfrak{h}$ ) choose an element $X_{\alpha} \neq 0$ belonging to the root $\alpha$. Let $Q$ be the set of all roots $\alpha$ such that $X_{\alpha} \in m_{\text {l }}$. We 
can choose a fundamental system $\left(\alpha_{1}, \cdots, \alpha_{r}\right)$ of roots in $Q$ so that $\alpha_{1}, \cdots, \alpha_{r}$ are linearly independent and every root in $Q$ can be written in the form $d_{1} \alpha_{1}+\cdots+d_{r} \alpha_{r}$ where $d_{1}, \cdots, d_{r}$ are integers which are either all nonnegative or all nonpositive. If $a \in A \cap K$, it is obvious that $\operatorname{Ad}(a) X_{\alpha}=c_{\alpha} X_{\alpha}$ $(\alpha \in Q)$ where $c_{\alpha}$ are unimodular complex numbers. Since $\left[X_{\alpha}, X_{-\alpha}\right] \in \mathfrak{h}$, $c_{-\alpha}=c_{\alpha}^{-1}(\alpha \in Q)$. We can choose $H \in \mathfrak{h}_{0} \cap \mathfrak{f}_{0}$ such that $c_{\alpha_{i}}=e^{\alpha_{i}(H)} i=1, \cdots, r$. Then $a_{0}=\exp H \in \bar{A}$ and $\operatorname{Ad}\left(a_{0}^{-1} a\right)$ leaves $X_{\alpha_{i}}, X_{-\alpha_{i}}(1 \leqq i \leqq r)$ fixed. But these elements together with $\mathfrak{h} \cap \mathfrak{f}$ generate the Lie algebra $\mathfrak{m}_{\mathfrak{t}}$, and so it follows that $a_{0}^{-1} a \in \bar{B}$. Hence $A \cap K=\bar{B} \bar{A}$. Moreover $\bar{A} \subset \bar{M}$ and therefore $M=\bar{M} A$ $=\bar{M} \bar{B} A_{+}$and $M \cap K=\bar{M} \bar{B}$. Since $\bar{A}$ contains the center of $\bar{M}$, it is now clear that $\bar{B}$ is the center of $\bar{M} \bar{B}$. Therefore

$$
M \cap K=\bar{M} \bar{B}=\bigcup_{m \in \bar{M}} m(\bar{A} \bar{B}) m^{-1}=\bigcup_{m \in \bar{M}}(A \cap K) m^{-1} .
$$

On the other hand it is obvious that $\bar{B} A_{+}$lies in the center of $M$. Conversely if $z$ is in the center of $M, z \in A$ since $A$ is maximal abelian. Hence $z=a a_{+}$ where $a \in A \cap K$ and $a_{+} \in A_{+}$. Then $a$ is in the center of $M \cap K$ and therefore $a \in \bar{B}$. This completes the proof of the lemma.

Let $H_{1}, \cdots, H_{p}$ be a base for $\mathfrak{h}_{0} \cap \mathfrak{p}_{0}$ over $R$. We extend it to a base $\left(H_{1}, \cdots, H_{p}, H_{p+1}, \cdots, H_{l}\right)$ for $\mathfrak{h}_{0} \cap \mathfrak{p}_{0}+(-1)^{1 / 2}\left(\mathfrak{h}_{0} \cap \mathfrak{f}_{0}\right)$ over $R$ and introduce the lexicographic order among the roots corresponding to this base (see $[6(\mathrm{f}), \S 2])$. Let $P$ be the set of all positive roots under this order. Then $P$ is the union of three disjoint subsets $P_{+}, P_{0}, P_{-}$defined as follows. $P_{+}$is the set of those roots $\alpha \in P$ which do not vanish identically on $\mathfrak{h} \cap \mathfrak{p}$. If $\alpha$ is a positive root which is not in $P_{+}$, it is clear that $X_{\alpha}$ and $\theta\left(X_{\alpha}\right)$ both belong to $\alpha$ and therefore since $\theta^{2}$ is the identity, $\theta\left(X_{\alpha}\right)= \pm X_{\alpha}$. Then $\alpha$ lies in $P_{0}$ or $P_{-}$according as $X_{\alpha} \in \mathfrak{p}$ or $X_{\alpha} \in \mathfrak{f}$. Put $\mathfrak{m}=\mathfrak{m}_{\mathfrak{t}}+\mathfrak{h} \cap \mathfrak{p}$ and

$$
q=\sum_{\alpha \in P_{+}}\left(C X_{\alpha}+C X_{-\alpha}\right)+\sum_{\alpha \in P_{0}}\left(C X_{\alpha}+C X_{-\alpha}\right) \text {. }
$$

Then $\mathfrak{q}$ is exactly the set of those elements of $\mathfrak{g}$ which are orthogonal to $\mathfrak{m}$ with respect to the bilinear form $B(X, Y)$. Since $\mathfrak{m} \cap g_{0}$ is the Lie algebra of $M$, it follows that $q$ is invariant under $\operatorname{Ad}(m)(m \in M)$. Let $I$ be the identity mapping of $\mathfrak{g}$ and $(\operatorname{Ad}(m)-I)_{\mathfrak{q}}$ the restriction of $\operatorname{Ad}(m)-I(m \in M)$ on $\mathfrak{q}$. We denote by ' $M$ the set of those elements $m \in M$ for which det (Ad $(m)-I)_{\text {a }}$ $\neq 0$. Put $M^{\prime}=M \cap G^{\prime}$ and $A^{\prime}=A \cap G^{\prime}$. It is obvious that ' $M \supset M^{\prime}$.

Lemma 8. $A^{\prime}$ is dense in $A$.

For each root $\alpha$ we can define a character $\xi_{\alpha}$ of $A$ by Ad $(a) X_{\alpha}=\xi_{\alpha}(a) X_{\alpha}$ $(a \in A)$. Select an element $H \in \mathfrak{h}_{0}$ such that $\alpha(H) \neq 0$ for every root $\alpha$. Then if $a \in A$, it is obvious that $\xi_{\alpha}(a \exp t H)=\xi_{\alpha}(a) e^{t_{\alpha}(H)} \neq 1$ for every root $\alpha$, provided $t$ is a sufficiently small positive number. This proves our assertion.

Corollary. Both ' $M$ and $M$ ' are open and dense in $M$. 
Since det $(\operatorname{Ad}(m)-I)_{\mathrm{a}}$ is an analytic function of $m$ on $M$, the openness of ' $M$ is obvious. Moreover $G^{\prime}$ being open in $G, M^{\prime}$ is open $\left(^{5}\right)$ in $M$. On the other hand

$$
M=\bigcup_{m \in \bar{M}} m A m^{-1} \text { and } M^{\prime}=\bigcup_{m \in \bar{M}} m A^{\prime} m^{-1} .
$$

Therefore since $A^{\prime}$ is dense in $A$ and ' $M \supset M^{\prime}$ 'our assertion follows.

Lemma 9. There exists a finite number of connected components $A_{1}^{\prime}, \cdots, A_{s}^{\prime}$ of $A^{\prime}$ such that $A^{\prime}=\bigcup_{i=1}^{s} Z A_{i}^{\prime}$.

For the purpose of this lemma, we can obviously replace $G$ by $G / Z$ and thus assume that $K$ is compact. Then $\bar{A}$ being defined as in Lemma 7 , $(A \cap K) / \bar{A}$ is both discrete and compact and therefore it is finite. Put $A_{0}=\bar{A} A_{+}$. Then it follows from Lemma 7 that every connected component of $A$ is of the form $a_{0} A_{0}\left(a_{0} \in A \cap K\right)$ and so there is only a finite number of such components. Define the characters $\xi_{\alpha}$ of $A$ as above. Since $\left[X_{\alpha}, X_{-\alpha}\right] \in \mathfrak{h}$, it is obvious that $\xi_{-\alpha}=\xi_{\alpha}^{-1}$. Select complex numbers $c_{\alpha}$ such that $\xi_{\alpha}\left(a_{0}\right)=e^{c_{\alpha}}$ $(\alpha \in P)$. Since $A \cap K$ is compact, $c_{\alpha}$ are all purely imaginary. Let $r$ be any integer and $\alpha$ a root in $P$. Then by $\sigma_{\alpha, r}$ we denote the hyperplane in $\mathfrak{h}_{0}$ consisting of all elements $H$ such that $\alpha(H)=-c_{\alpha}+(-1)^{1 / 2} 2 \pi r$. It is clear that any compact subset of $\mathfrak{h}_{0}$ meets only a finite number of these hyperplanes. Let $\mathfrak{h}_{0}^{\prime}$ be the complement in $\mathfrak{h}_{0}$ of the union of all these hyperplanes. Then every point in the real Euclidean space $\mathfrak{h}_{0}$ has a neighborhood $V$ such that $V \cap \mathfrak{h}_{0}^{\prime}$ decomposes only into a finite number of connected components. From this we conclude immediately that if $\omega$ is a compact subset of $\mathfrak{h}_{0}$, there exists a neighborhood $V$ of $\omega$ for which $V \cap \mathfrak{h}_{0}^{\prime}$ has only a finite number of connected components. Now $\bar{A}$ being compact, we can choose a compact set $\omega_{\mathrm{t}_{0}}$ in $\mathfrak{h}_{0} \cap \mathfrak{f}_{0}$ such that $\bar{A} \subset \exp \omega_{\mathfrak{t}_{0}}$. Put $\omega^{\prime}=\mathfrak{h}_{0}^{\prime} \cap\left(\mathfrak{h}_{0} \cap \mathfrak{p}_{0}+\omega \mathfrak{t}_{0}\right)$ and suppose $H^{\prime} \in \omega^{\prime}$. Then $H^{\prime}=H+H_{0}$ where $H \in \mathfrak{h}_{0} \cap \mathfrak{p}_{0}, H_{0} \in \omega_{t_{0}}$. Consider $H_{t}^{\prime}=t H+H_{0}(t>0)$. We claim $H_{t}^{\prime} \in \omega^{\prime}$. For otherwise $\alpha\left(H_{t}^{\prime}\right)=-c_{\alpha}+(-1)^{1 / 2} 2 \pi r$ for some $\alpha \in P$ and some integer $r$. But

$$
\alpha\left(H_{t}^{\prime}\right)=t \alpha(H)+\alpha\left(H_{0}\right)
$$

and $\alpha(H)$ is real while $\alpha\left(H_{0}\right)$ is purely imaginary. Therefore since $c_{\alpha}$ is also purely imaginary, the above relation is possible only if $\alpha(H)=0$. But in that case

$$
\alpha\left(H^{\prime}\right)=\alpha\left(H_{t}^{\prime}\right)=-c_{\alpha}+(-1)^{1 / 2} 2 \pi r
$$

which however is impossible since $H^{\prime} \in \mathfrak{h}_{0}^{\prime}$. Let $V$ be a neighborhood of $\omega_{\mathfrak{t}_{0}}$ in $\mathfrak{h}_{0}$ such that $V \cap \mathfrak{h}_{0}^{\prime}$ has only a finite number of connected components. Then the above result shows that every point of $\omega^{\prime}$ can be joined to a point

(5) We note that $M$ is a closed subgroup of $G$ and therefore the natural topology on $M$ as a Lie group coincides with the one induced by $G$ (see Chevalley [2]). A similar statement holds for $A$. 
in $V$ by a line lying entirely in $\omega^{\prime}$. Therefore $\omega^{\prime}$ also does not have more than a finite number of connected components. On the other hand it is obvious that the image of $\omega^{\prime}$ under the mapping $H \rightarrow a_{0} \exp H\left(H \in \omega^{\prime}\right)$ is exactly $A^{\prime} \cap a_{0} A_{0}$. This proves that no component of $A$ contains more than a finite number of components of $A^{\prime}$. Therefore since $A$ has only a finite number of components the same holds for $A^{\prime}$.

COROLLARY. There exists a finite number of connected components $G_{1}^{\prime}, \cdots$, $G_{r}^{\prime}$ of $G^{\prime}$ such that $G^{\prime}=\bigcup_{i=1}^{r} Z G_{i}^{\prime}$.

This follows immediately from Lemmas 5 and 9.

Lemma 10. Let $A^{*}$ denote the normalizer of $A$ in $G$. Then $A^{*}=\left(A^{*} \cap K\right) A_{+}$, the factor group $A^{*} / A$ is finite and $a^{*} M a^{*-1}=M$ for any $a^{*} \in A^{*}$. Moreover if $x$ is an element in $G$ such that $x m x^{-1} \in M$ for some $m \in \in^{\prime} M$, then $x \in A^{*} M$.

Since $\theta(A)=A$ it is obvious that $\theta\left(A^{*}\right)=A^{*}$. Now suppose $k p \in A^{*}$ $\left(k \in K, p \in \exp \mathfrak{p}_{0}\right)$. Then $p^{2}=(\theta(k p))^{-1} k p \in A^{*}$ and therefore Ad $\left(p^{2}\right) \mathfrak{h}_{0}=\mathfrak{h}_{0}$. But an element $H \in \mathfrak{h}_{0}$ belongs to $\mathfrak{p}_{0}$ (or $\mathfrak{l}_{0}$ ) if and only if all the eigenvalues of ad $H$ are real (or purely imaginary). This implies that $\mathrm{Ad}\left(p^{2}\right)$ leaves both $\mathfrak{h}_{0} \cap \mathfrak{p}_{0}$ and $\mathfrak{h}_{0} \cap \mathfrak{f}_{0}$ invariant. Choose $Y \in \mathfrak{p}_{0}$ such that $p=\exp Y$. Since $\left[\mathfrak{p}_{0}, \mathfrak{p}_{0}\right]$ $\subset \mathfrak{l}_{0}$ and $\left[\mathfrak{p}_{0}, \mathfrak{l}_{0}\right] \subset \mathfrak{p}_{0}$, we conclude that

$$
\left\{\operatorname{Ad}\left(p^{2}\right)-\operatorname{Ad}\left(p^{-2}\right)\right\} H=0 \quad\left(H \in \mathfrak{h}_{0}\right) .
$$

But as we have seen during the proof of Lemma 7, this is possible only if $(\operatorname{ad} Y) H=0$ for all $H \in \mathfrak{h}_{0}$. This means however that $Y \in \mathfrak{h}_{0} \cap \mathfrak{p}_{0}$ and therefore $p \in A_{+}$and $k \in A^{*} \cap K$. Moreover $\mathfrak{m}_{0}$ being the centralizer of $\mathfrak{h}_{0} \cap \mathfrak{p}_{0}$ in $\mathfrak{l}_{0}$, it is obvious that $\operatorname{Ad}(k) \mathfrak{m}_{\mathfrak{t}_{0}}=\mathfrak{m}_{\mathfrak{t}_{0}}$ and therefore $k \bar{M} k^{-1}=\bar{M}$. Since $M=\bar{M} A$, this proves that $a^{*} M a^{*-1}=M$ for any $a^{*} \in A^{*}$. Also $\mathfrak{h}_{0}$ being its own normalizer in $\mathfrak{g}_{0}$, it follows that $A^{*}$ and $A$ have the same connected component of 1 . Therefore since $Z \subset A \cap K$ and $K / Z$ is compact, $A^{*} / A \simeq\left(A^{*} \cap K\right) / A \cap K$ is both discrete and compact and so it must be finite.

Now suppose $x m x^{-1} \in M$ for some $m \in \in^{\prime} M$ and $x \in G$. Then $m=m_{1} a m_{1}^{-1}$ where $a \in^{\prime} M \cap A=' A$ and $m_{1} \in M$. Similarly $x m x^{-1}=m_{2} a^{\prime} m_{2}^{-1} \quad\left(a^{\prime} \in A\right.$, $\left.m_{2} \in M\right)$. Hence if $y=m_{2}^{-1} x m_{1}$, it is sufficient to prove that $y \in A^{*} M$. But $a \in y^{-1} A y$ and therefore it is obvious that $\operatorname{Ad}(a) X=X$ if $X \in \operatorname{Ad}\left(y^{-1}\right) \mathfrak{h}_{0}$. On the other hand since $a \in{ }^{\prime} A$, Ad $(a) X \neq X\left(X \in \mathfrak{g}_{0}\right)$ unless $X \in \mathfrak{h}_{0} \cap \mathfrak{p}_{0}+\mathfrak{m}_{\mathfrak{t}_{0}}=\mathfrak{m}_{0}$. Therefore Ad $\left(y^{-1}\right) \mathfrak{h}_{0} \subset \mathfrak{m}_{0}$. Moreover Ad $(\bar{M})$ being compact, any two maximal abelian subalgebras of $m_{0}$ are conjugate under $\bar{M}$. Therefore we can select $m_{3} \in \bar{M}$ such that Ad $\left(y^{-1}\right) \mathfrak{h}_{0}=\operatorname{Ad}\left(m_{3}\right) \mathfrak{h}_{0}$. Then from Lemma 3, $y m_{3} \in A^{*}$ and therefore $y \in A^{*} M$.

3. Quasi-regular elements. Let $Z_{x}$ and $\mathfrak{h}_{x}$ be the centralizers in $G$ and $\mathfrak{g}_{0}$ respectively of an element $x \in G$. We shall say that $x$ is quasi-regular if (1) $\mathfrak{h}_{x}$ contains a regular element and (2) there exists a closed normal abelian subgroup $A$ of $Z_{x}$ such that $Z_{x} / A$ is compact. It follows from the corollary to 
Lemma 3 that every regular element is quasi-regular. Now suppose $x$ is quasi-regular and $H$ is a regular element in $\mathfrak{h}_{x}$. Then $x$ lies in the centralizer of $H$ in $G$ and therefore from Lemmas 3 and $6, x$ is conjugate to an element of some $A_{i}(1 \leqq i \leqq r)$. Thus we have obtained the following result.

LemMa 11. Let ' $G$ be the set of all quasi-regular elements in $G$ and put $' A_{i}={ }^{\prime} G \cap A_{i}(1 \leqq i \leqq r)$ where $A_{i}$ have the same meaning as in Lemma 6. Then $' G=\bigcup_{i=1}^{r} \bigcup_{x \in G} x^{\prime} A_{i} x^{-1}$.

Let us now use the notation of Lemma 7 and define ' $M$ as in the corollary to Lemma 8. Our object is to show that ' $G \cap M={ }^{\prime} M$. Suppose $a_{0}$ is a quasiregular element in $A$. Define $\xi_{\alpha}(\alpha \in P)$ as in the proof of Lemma 8 and let $\mathfrak{n}$ be the centralizer of $a_{0}$ in $\mathrm{g}$. Then if $Q$ is the set of those roots $\alpha \in P$ for which $\xi_{\alpha}\left(a_{0}\right)=1, \mathfrak{n}=\mathfrak{h}+\sum_{\alpha \in Q}\left(C X_{\alpha}+C X_{-\alpha}\right)$. Moreover since $a_{0}$ lies in $G, \mathfrak{n}$ is the complexification of $\mathfrak{n}_{0}=\mathfrak{n} \cap \mathfrak{g}_{0}$ and $\mathfrak{n}_{0}$ is the Lie algebra of $Z_{a_{0}}$. Now let $y \in Z_{a_{0}}$. Then it follows from the definition of quasi-regularity that all the eigenvalues of the restriction of $\mathrm{Ad}(y)$ on $\mathfrak{n}$ are unimodular complex numbers. Therefore since $A \subset Z_{a_{0}}$, we conclude that $\left|\xi_{\alpha}(a)\right|=1$ for all $a \in A$ and $\alpha \in Q$. This shows that every root in $Q$ takes only purely imaginary values on $\mathfrak{h}_{0}$. On the other hand since all roots take only real values on $\mathfrak{h}_{0} \cap \mathfrak{p}_{0}$, it follows that $Q \subset P_{0} \cup P_{-}$ and therefore $\theta(\mathfrak{n})=\mathfrak{n}$. Furthermore it is obvious from the above expression for $\mathfrak{n}$ that it is reductive (see Koszul [9]) and so any abelian ideal of $\mathfrak{n}$ must be contained in $\mathfrak{h}$. Hence in view of the quasi-regularity of $a_{0}, \mathfrak{n}_{0}^{\prime}=\left[\mathfrak{n}_{0}, \mathfrak{n}_{0}\right]$ must be a compact semisimple Lie algebra over $R$. On the other hand $\theta\left(\mathfrak{n}_{0}^{\prime}\right)$ $=\mathfrak{n}_{0}^{\prime}$ and therefore $\mathfrak{n}_{0}^{\prime}=\mathfrak{n}_{0}^{\prime} \cap \mathfrak{l}_{0}+\mathfrak{n}_{0}^{\prime} \cap \mathfrak{p}_{0}$. But if $X \in \mathfrak{n}_{0}^{\prime} \cap \mathfrak{p}_{0}$ all the eigenvalues of ad $X$ are real which, from the compactness and semisimplicity of $\mathfrak{n}_{0}^{\prime}$, is impossible unless $X=0$. This shows that $\mathfrak{n}_{0}^{\prime} \subset \mathfrak{l}_{0}$ and therefore $\mathfrak{l} \supset[\mathfrak{h}, \mathfrak{n}]$ $\supset \sum_{\alpha \in Q}\left(C X_{\alpha}+C X_{-\alpha}\right)$. Hence $Q \subset P_{-}$and so $a_{0} \in A \cap^{\prime} M$. Conversely if $m \in \in^{\prime} M, Z_{m}$ is contained in $A^{*} M$ and $A_{+} Z$ is a closed normal subgroup of $A^{*} M$ such that $A^{*} M / A_{+} Z$ is compact (see Lemma 10). Moreover since $m$ is conjugate to some element of $A$ (Lemma 7), the centralizer of $m$ in $g_{0}$ contains a regular element. This proves that $m$ is quasi-regular. Therefore $A \cap \cap^{\prime} G=A \cap{ }^{\prime} M$ and since $M=\bigcup_{m \in M} m A m^{-1}$, it follows that ' $M=M \cap^{\prime} G$. Thus we have the following lemma.

Lemma 12. ' $M$ coincides with the set of quasi-regular elements in $M$.

COROLLARY. ' $G$ is an open subset of $G$ and there exists a finite number of connected components $G_{1}, \cdots, G_{N}$ of ' $G$ such that ${ }^{\prime} G=\bigcup_{i=1}^{N} Z G_{i}$.

Consider the mapping $(x, m) \rightarrow x m x^{-1}\left(x \in G, m \in \mathcal{C}^{\prime} M\right)$. Since (Ad $\left(m^{-1}\right)$ $-I) \mathfrak{q}=\mathfrak{q}$, every element in $\mathfrak{g}$ can be written in the form $X+\left(\operatorname{Ad}\left(m^{-1}\right)-I\right) Y$ $(X \in \mathfrak{m}, Y \in \mathfrak{q})$. Hence this mapping is everywhere open and therefore $\bigcup_{x \in G} x^{\prime} M x^{-1}$ is an open set in $G$. But ' $M=\bigcup_{m \in M} m^{\prime} A m^{-1}$ where ' $A=A \cap^{\prime} G$. Hence $U_{x \in G} x^{\prime} A x^{-1}$ is an open set and we conclude from Lemma 11 that ' $G$ is also open. Now $G^{\prime}$ being the set of regular elements in $G,{ }^{\prime} G \supset G^{\prime}$ and $G^{\prime}$ is 
dense in $G$. Hence every connected component of ' $G$ contains some connected component of $G^{\prime}$. Define $G_{i}^{\prime} 1 \leqq i \leqq r$ as in the corollary to Lemma 9 and select a connected component $G_{i}$ of ' $G$ containing $G_{i}^{\prime}$. Then $\bigcup_{i=1}^{r} Z G_{i} \supset G^{\prime}$ and therefore every connected component of ' $G$ must intersect and therefore coincide with $z G_{i}$ for some $z \in Z$ and some $i$. Hence ' $G=\bigcup_{i=1}^{r} Z G_{i}$.

4. Some general facts about differential operators. Let $V$ be a locally connected topological space on each connected component of which there is defined the structure of a differentiable manifold of class $C^{\infty}$ (or of an analytic manifold) and dimension $n$. Under these conditions we say that $V$ is a differentiable (or analytic) manifold of dimension $n$. This definition differs from the usual one in not assuming that $V$ is connected. It has the advantage that every open subset $U$ of $V$ may now be regarded as an open submanifold of $V$. Let $C_{c}^{\infty}(U)$ denote the set of all complex-valued functions on $V$ which are everywhere differentiable and which vanish outside a compact set contained in $U$. Then $C_{c}^{\infty}(U)$ is a vector space over $C$ and a distribution $T$ on $U$ is a linear function on this vector space satisfying certain conditions of continuity (see Schwartz [11]). Let $D$ be a linear mapping of $C_{c}^{\infty}(V)$ into itself and $p$ a point in $V$. We say that $D$ is a differential operator at $p$ if there exists a coordinate system $\left(t_{1}, \cdots, t_{n}\right)$ valid on some open neighborhood $U$ of $p$ and having the following property. Let $\partial_{i}$ denote the operators $\partial / \partial t_{i}(1 \leqq i \leqq n)$ of partial differentiation. We require that there should exist a finite set of indefinitely differentiable functions $g_{i_{1} i_{2}} \ldots i_{q}\left(1 \leqq i_{1}, \cdots, i_{q} \leqq n, 0 \leqq q \leqq r\right)$ on $U$ such that for every $f \in C_{c}^{\infty}(U)$,

$$
D f=\sum_{0 \leqq q \leqq r} \sum_{1 \leqq i_{1}, \cdots, i_{q} \leqq n} g_{i_{1} i_{2} \cdots i_{q}} \partial_{i_{1}} \partial_{i_{2}} \cdots \partial_{i_{q}} f
$$

on $U$ and $D f=0$ outside $U$. (In case $q=0, \partial_{i_{1}} \partial_{i_{2}} \cdots \partial_{i_{q}} f$ should be interpreted to mean $f_{*}$ ) If $D$ is a differential operator at every point of $V$, we say simply that it is a differential operator (on $V$ ). Clearly the differential operators form a subalgebra of the algebra of all endomorphisms of $C_{c}^{\infty}(V)$. In particular if $g$ is an indefinitely differentiable function on $V$ the mapping $f \rightarrow g f\left(f \in C_{c}^{\infty}(V)\right)$ is a differential operator which we again denote by $g$.

Let $C^{\infty}(V)$ denote the space of all indefinitely differentiable functions on $V$. Then any differential operator $D$ also defines an endomorphism of $C^{\infty}(V)$. In fact if $f \in C^{\infty}(V)$ and $p$ is a point in $V,(D f)(p)=(D g)(p)$ where $g$ is any function in $C_{c}^{\infty}(V)$ such that $f-g$ is identically zero on some neighborhood of $p$. It would of ten be convenient to denote the value of $D f$ at $p$ by $f(p, D)$. $V$ being an analytic manifold, we say that $D$ is analytic if $D f$ is analytic at a point $p$ whenever $f$ is analytic at $p\left(f \in C^{\infty}(V)\right)$.

Now suppose $D_{1}, D_{2}$ are differential operators on two manifolds $V_{1}$ and $V_{2}$ respectively. Then $D=D_{1} \times D_{2}$ is a differential operator on the product manifold $V_{1} \times V_{2}$ (see Schwartz [11]). In fact if $f_{1} \in C^{\infty}\left(V_{1}\right), f_{2} \in C^{\infty}\left(V_{2}\right)$ and $g\left(p_{1}, p_{2}\right)=f_{1}\left(p_{1}\right) f_{2}\left(p_{2}\right)\left(p_{1} \in V_{1}, p_{2} \in V_{2}\right)$ then 


$$
(D g)\left(p_{1}, p_{2}\right)=f_{1}\left(p_{1}, D_{1}\right) f_{2}\left(p_{2}, D_{2}\right) \text {. }
$$

We shall sometimes denote the value of $D h\left(h \in C^{\infty}\left(V_{1} \times V_{2}\right)\right)$ at $\left(p_{1}, p_{2}\right)$ $\in V_{1} \times V_{2}$ by $h\left(p_{1}, D_{1} ; p_{2}, D_{2}\right)$.

Let $L$ be a Lie group and let $\mathfrak{I}_{0}$ denote its Lie algebra over $R$.Then if $L^{\prime}$ is a nonempty open subset of $L$, every $X \in \mathfrak{l}_{0}$ defines a differential operator on $L^{\prime}$. In fact if $f \in C_{c}^{\infty}\left(L^{\prime}\right), X f$ is given by $f(x, X)=\{d f(x \exp t X) / d t\}_{t=0}$ $\left(x \in L^{\prime}, t \in R\right)$. It is well known (see Chevalley [2]) that $[X, Y] f=X(Y f)$ $-Y(X f)\left(X, Y \in \mathfrak{I}_{0}, f \in C_{c}^{\infty}\left(L^{\prime}\right)\right)$ and therefore we get a representation of $\mathfrak{l}_{0}$ on $C_{c}^{\infty}\left(L^{\prime}\right)$. Let $\mathfrak{l}$ be the complexification of $\mathfrak{l}_{0}$ and $\mathfrak{l}$ the universal enveloping algebra of $\mathfrak{l}[6(\mathrm{a})]$. We may extend this representation (uniquely) to a representation of $\mathfrak{R}$ on $C_{c}^{\infty}\left(L^{\prime}\right)$ and therefore every element $b \in \mathfrak{R}$ defines a differential operator $f \rightarrow b f\left(f \in C_{c}^{\infty}\left(L^{\prime}\right)\right)$ on $L^{\prime}$. Let $b f(x)$ denote the value of $b f$ at $x \in L^{\prime}$.

Lemma $\left({ }^{6}\right)$ 13. Let $x_{0}$ be $a$ point in $L^{\prime}$ and $b$ an element in $\&$. If $b f\left(x_{0}\right)=0$ for every $f \in C_{c}^{\infty}\left(L^{\prime}\right)$ then $b=0$.

Let $X_{1}, \cdots, X_{n}$ be a base for $\mathfrak{l}_{0}$ over $R$. We can choose a positive number $\epsilon$ and an open neighborhood $U$ of $x_{0}$ in $L^{\prime}$ with the following property. If $Q$ is the open cube consisting of all points $t=\left(t_{1}, \cdots, t_{n}\right)$ in $R^{n}$ with $|t|$ $=\max _{i}\left|t_{i}\right|<\epsilon$, the mapping

$$
t \rightarrow x_{0} \exp X(t)
$$

where $X(t)=t_{1} X_{1}+\cdots+t_{n} X_{n}$, is a one-one regular mapping of $Q$ onto $U$. Then it is obvious that if $P(t)$ is any polynomial in $\left(t_{1}, \cdots, t_{n}\right)$ with complex coefficients, there exists a function $f_{P} \in C_{c}^{\infty}\left(L^{\prime}\right)$ such that $f_{P}\left(x_{0} \exp X(t)\right)=P(t)$ for $|t| \leqq \epsilon / 2$. In particular $f_{P}$ is analytic at $x_{0}$ and its Taylor expansion

$$
f_{P}\left(x_{0} \exp X(t)\right)=\sum_{m \geqq 0} \frac{1}{m !}(X(t))^{m} f\left(x_{0}\right)
$$

is valid if $|t|$ is sufficiently small. Now $M$ being an ordered set $\left(m_{1}, \cdots, m_{n}\right)$ of $n$ non-negative integers, put $t^{M}=t_{1}^{m_{1}} \cdots t_{n}^{m_{n}},|M|=m_{1}+\cdots+m_{n}$ and let $X(M)$ denote the coefficient of $t^{M}$ in $(|M| !)^{-1}(X(t))^{|M|}$. Then

$$
P(t)=f\left(x_{0} \exp X(t)\right)=\sum_{m \geqq 0} \sum_{|M|=m} t^{M} X(M) f_{P}\left(x_{0}\right)
$$

provided $|t|$ is sufficiently small. Hence if $P_{M}$ is the coefficient of $t^{M}$ in $P(t)$, $P_{M}=X(M) f_{P}\left(x_{0}\right)$. On the other hand the elements $X(M)$ form a base for \& over $C$ (see [6(a) ]). Therefore $b=\sum_{M} a(M) X(M)$ where $a(M) \in C$ and the sum is finite. If $b \neq 0$ we can choose $M_{0}$ such that $a\left(M_{0}\right) \neq 0$. Now put $P=t^{M_{0}}$. Then

$$
b f_{P}\left(x_{0}\right)=a\left(M_{0}\right) \neq 0 .
$$

(6) This lemma is attributed to Schwartz by Godement [5]. 
This proves our assertion.

Corollary. Let $D$ be a differential operator on $L^{\prime}$ and $x_{0}$ a point in $L^{\prime}$. Then there exists exactly one element $b \in \mathbb{R}$ such that $f\left(x_{0}, D\right)=b f\left(x_{0}\right)$ for all $f \in C_{c}^{\infty}\left(L^{\prime}\right)$.

We keep to the notation introduced above. Since $t_{1}, \cdots, t_{n}$ form a coordinate system on $U$ under the mapping $t \rightarrow x_{0} \exp X(t)$ and

$$
m_{1} ! m_{2} ! \cdots m_{n} ! X(M) f\left(x_{0}\right)=\left\{\frac{\partial^{m_{1}+\cdots+m_{n}}}{\partial t_{1}^{m_{1}} \cdots \partial t_{n}^{m_{n}}} f\left(x_{0} \exp X(t)\right)\right\}_{t=0}
$$

for $M=\left(m_{1}, \cdots, m_{n}\right)$ and $f \in C_{c}^{\infty}\left(L^{\prime}\right)$, it follows from the definition of a differential operator that there exists an element $b=\sum_{M} a(M) X(M) \in \mathbb{R}$ such that $f\left(x_{0}, D\right)=b f\left(x_{0}\right)$ for all $f \in C_{c}^{\infty}\left(L^{\prime}\right)$. On the other hand the uniqueness of $b$ is an immediate consequence of the above lemma.

We call the element $b$ of the above corollary the local expression of $D$ at $x_{0}$. Lemma 13 shows that the representation of $\mathscr{R}$ on $C_{c}^{\infty}\left(L^{\prime}\right)$ is faithful and so we may identify elements of $\mathbb{R}$ with the corresponding differential operators on $L^{\prime}$. In this way $\mathfrak{R}$ becomes a subalgebra of the algebra of all differential operators on $L^{\prime}$. Moreover if $X \in \mathfrak{l}_{0}, X$ is an analytic differential operator on $L^{\prime}$ (see Chevalley [2]) and therefore the same holds for every $b \in \mathbb{R}$.

Let $x \rightarrow \operatorname{Ad}(x)$ denote the adjoint representation of $L$ on $\mathfrak{l}$. We extend Ad $(x)$ to an automorphism of $\mathfrak{R}$ over $C$ and write $a^{x}=\operatorname{Ad}(x) a(a \in \mathfrak{R}, x \in L)$ whenever it is convenient to do so. Now suppose $L^{\prime}$ is an invariant set which means that $x L^{\prime} x^{-1}=L^{\prime}$ for all $x \in L$. Then for any function $f$ on $L^{\prime}$, define $f^{x}(x \in L)$ to be the function $y \rightarrow f\left(x^{-1} y x\right)\left(y \in L^{\prime}\right)$. We say that $f$ is invariant if $f=f^{x}$ for all $x \in L$. It is clear that $f \rightarrow f^{x}\left(f \in C_{c}^{\infty}\left(L^{\prime}\right)\right)$ is a linear isomorphism of $C_{c}^{\infty}\left(L^{\prime}\right)$ onto itself and if $a \in R, f^{x}(y, a)=f\left(x^{-1} y x\right.$, Ad $\left.\left(x^{-1}\right) a\right)\left(x \in L, y \in L^{\prime}\right)$. We shall always denote by $I_{c}^{\infty}\left(L^{\prime}\right)$ and $I^{\infty}\left(L^{\prime}\right)$ respectively the sets of invariant functions in $C_{c}^{\infty}\left(L^{\prime}\right)$ and $C^{\infty}\left(L^{\prime}\right)$. $D$ being any differential operator, the mapping $f \rightarrow\left(D\left(f^{x^{-1}}\right)\right)^{x}\left(f \in C_{c}^{\infty}\left(L^{\prime}\right)\right)$ is also a differential operator which we denote by $D^{x}$. In particular if $D \in \mathbb{R}, D^{x}=\operatorname{Ad}(x) D$ and so this coincides with the definition of $a^{x}(a \in \mathfrak{R})$ given above. We say that $D$ is invariant if $D^{x}=D$ for all $x \in L$. Let $D_{y}\left(y \in L^{\prime}\right)$ denote the local expression of $D$ at $y$.

Lemma 14. If $D$ is a differential operator on $L^{\prime}$,

$$
\operatorname{Ad}(x) D_{y}=\left(D^{x}\right)_{x y x^{-1}} \quad\left(x \in L, y \in L^{\prime}\right) .
$$

Therefore $D$ is invariant if and only if $D_{x y x^{-1}}=\operatorname{Ad}(x) D_{y}$ for all $x \in L$ and $y \in L^{\prime}$.

If $f \in C_{c}^{\infty}\left(L^{\prime}\right)$, it follows from the definition of $D^{x}$ that

$$
\begin{aligned}
f\left(x y x^{-1}, D^{x}\right) & =f^{x-1}(y, D)=f^{x-1}\left(y, D_{y}\right) \\
& =f\left(x y x^{-1}, \operatorname{Ad}(x) D_{y}\right) .
\end{aligned}
$$


Hence the lemma.

Similarly if $\tau$ is a distribution on $L^{\prime}$, the mapping $f \rightarrow \tau\left(f^{x^{-1}}\right)\left(f \in C_{c}^{\infty}\left(L^{\prime}\right)\right)$ is also a distribution which we denote by $\tau^{x}(x \in L)$. Again $\tau$ is said to be invariant if $\tau^{x}=\tau$ for all $x \in L$. Now suppose the group $L$ is unimodular so that its Haar measure $d x$ is both left- and right-invariant. Let $U$ be a (nonempty) open set in $L^{\prime}$ and $F$ a complex-valued function on $U$. We say that $F$ is locally summable if it is measurable with respect to the Haar measure and $\int_{\omega}|F(x)| d x$ $<\infty$ for any compact set $\omega$ contained in $U$. Corresponding to any such $F$, we define a distribution $\tau_{F}$ on $U$ as follows:

$$
\tau_{F}(f)=\int f(x) F(x) d x \quad\left(f \in C_{0}^{\infty}(U)\right) .
$$

If $\tau$ is a distribution (defined on some open set containing $U$ ) we say that $\tau=F$ on $U$ (or $\tau$ coincides with $F$ on $U$ ) if $\tau(f)=\tau_{F}(f)$ for every $f \in C_{c}^{\infty}(U)$. Let $D$ be a differential operator on $U$. Then there exists a uniquely determined differential operator $D^{*}$ on $U$ such that

$$
\int\left(D^{*} f\right) g d x=\int f(D g) d x \quad\left(f, g \in C_{c}^{\infty}(U)\right) .
$$

$D^{*}$ is called the adjoint of $D$. The mapping $D \rightarrow D^{*}$ is obviously an antiautomorphism of order 2 of the algebra of all differential operators on $U$. In particular if $g \in C^{\infty}(U), g^{*}=g$. Now if $\tau$ is a distribution and $D$ a differential operator on $U$, we define a distribution $D \tau$ by $(D \tau)(f)=\tau\left(D^{*} f\right)\left(f \in C_{c}^{\infty}(U)\right)$. In this way we get a representation of the algebra of differential operators on the space of distributions. $\tau$ is said to be an eigen-distribution of $D$ if $D \tau=c \tau$ where $c$ is a complex number. Let $\phi$ be the anti-automorphism of $\mathfrak{R}$ such that $\phi(X)=-X(X \in \mathfrak{l})$. Then it is obvious that $X^{*}=\phi(X)$ if $X \in \mathfrak{l}$ and therefore $b^{*}=\phi(b)$ for all $b \in \mathbb{R}$. Moreover if $U$ is an invariant set, it is easy to see that a differential operator $D$ on $U$ is invariant if and only if $D^{*}$ is invariant.

5. Differential operators on ' $M$. Now let us return to the notation of Lemma 7 and define ' $M$ as in the corollary to Lemma 8. Then ' $M$ is an open submanifold of the Lie group $M$. Let $\mathfrak{B}$ be the universal enveloping algebra of $\mathfrak{g}$ and $\mathfrak{M}$ the subalgebra of $\mathfrak{B}$ generated by $(1, \mathfrak{m})$ where

$$
\mathfrak{m}=\mathfrak{h}+\sum_{\alpha \in P_{-}}\left(C X_{\alpha}+C X_{-\alpha}\right) .
$$

Then $\mathfrak{M}$ is the universal enveloping algebra of $\mathfrak{m}$ (see Lemma 21 of [6(b)]) and therefore $\mathfrak{B}$ and $\mathfrak{M}$ are certain algebras of differential operators on $G$ and $M$ respectively. For any $b \in \mathfrak{B}$ let $L_{b}$ and $R_{b}$ respectively denote the linear mappings $a \rightarrow b a$ and $a \rightarrow a b(a \in \mathfrak{B})$ of $\mathfrak{B}$. $\phi$ being an indefinitely differentiable function on $G$, put $\Phi(x ; m)=\phi\left(x m x^{-1}\right)(x \in G, m \in M)$. Then $\Phi \in C^{\infty}(G \times M)$ 
and if $X_{1}, \cdots, X_{r} \in g_{0}$ and $H_{1}, \cdots, H_{s} \in \mathfrak{m}_{0}=\mathfrak{m} \cap \mathfrak{g}_{0}$, we have

$$
\begin{aligned}
& \Phi\left(x, X_{1} X_{2} \cdots X_{r} ; m, H_{1} H_{2} \cdots H_{s}\right) \\
& =\left\{\frac{\partial^{r+s}}{\partial u_{1} \cdots \partial u_{r} \partial t_{1} \cdots \partial t_{s}} \phi\left(x y(u) m m(t)(y(u))^{-1} x^{-1}\right)\right\}_{u_{i}=t_{j}=0}
\end{aligned}
$$

where $y(u)=\exp u_{1} X_{1} \cdots \exp u_{r} X_{r}$ and $m(t)=\exp t_{1} H_{1} \cdots \exp t_{s} H_{\text {。 }}$ $\left(u_{i}, t_{j} \in R\right)$. Therefore, as a simple induction shows,

$$
\Phi\left(x, X_{1} X_{2} \cdots X_{r} ; m, H_{1} H_{2} \cdots H_{s}\right)=\phi\left(x m x^{-1}, w^{x}\right)
$$

where

$$
w=\left(L_{\mathrm{Ad}\left(m^{-1}\right) X_{1}}-R_{X_{1}}\right) \cdots\left(L_{\mathrm{Ad}\left(m^{-1}\right) X_{r}}-R_{X_{r}}\right)\left(H_{1} \cdots H_{s}\right) .
$$

This suggests the lemma below. Let $\mathfrak{B} \times \mathfrak{M}$ denote the tensor product of $\mathfrak{B}$ and $\mathfrak{M}$ and $S(\mathfrak{q})$ and $S(\mathfrak{g})$ the symmetric algebras over $\mathfrak{q}$ and $\mathfrak{g}$ respectively (see [6(c), §2]). Then $S(\mathfrak{q}) \subset S(\mathfrak{g})$. Put $\mathfrak{Q}=\lambda(S(\mathfrak{q}))$ where $\lambda$ is the canonical mapping [6(c), p. 192] of $S(\mathrm{~g})$ onto $\mathfrak{B}$. Then if $m \in M$, it is obvious that Ad $(m) \mathfrak{Q}=\mathfrak{Q}$. Let $S_{r}(\mathfrak{g})$ be the space of those elements in $S(\mathfrak{g})$ which are homogeneous of degree $r$. We call an element $b \in \mathfrak{B}$ homogeneous of degree $r$ if $b \in \lambda\left(S_{r}(\mathfrak{g})\right)$ and of degree $\leqq r$ if $b \in \sum_{0 \leqq d \leqq r} \lambda\left(S_{d}(\mathfrak{g})\right)$. Similarly we say that an element in $\mathfrak{B} \times \mathfrak{M}$ is of total degree $\leqq r$ if it lies in the space $\sum_{d+e} \leqq r \mathfrak{B}_{d} \times \mathfrak{M}_{e}$ where $\mathfrak{B}_{d}$ and $\mathfrak{M}$ 。 respectively are the subspaces of $\mathfrak{B}$ and $\mathfrak{M}$.consisting of homogeneous elements of degrees $d$ and $e$.

Lemma 15. For each $m \in M$ there exists a unique linear mapping $\Gamma_{m}$ of $\mathfrak{B} \times \mathfrak{M}$ into $\mathfrak{B}$ such that $\Gamma_{m}(1 \times \nu)=\nu$ and

$$
\Gamma_{m}\left(X_{1} X_{2} \cdots X_{r} \times \nu\right)=\left(L_{\mathrm{Ad}\left(m^{-1}\right) X_{1}}-R_{X_{1}}\right) \cdots\left(L_{\mathrm{Ad}\left(m^{-1}\right) X_{r}}-R_{X_{r}}\right) \nu
$$

for all $X_{1}, \cdots, X_{r} \in \mathfrak{g}$ and $\nu \in \mathfrak{M}$. Moreover if $m \in \mathcal{C}^{\prime} M, \Gamma_{m}$ defines a one-one mapping of $\mathfrak{Q} \times \mathfrak{M}$ onto $\mathfrak{B}$.

The uniqueness of $\Gamma_{m}$ is obvious and so only the existence requires proof. Put

$$
\left.\gamma_{m}\left(X_{1}, \cdots, X_{r} ; \nu\right)=L_{\mathrm{Ad}\left(m^{-1}\right) X_{1}}-R_{X_{1}}\right) \cdots\left(L_{\mathrm{Ad}\left(m^{-1}\right) X_{r}}-R_{X_{r}}\right) \nu
$$

for $m \in M, X_{1}, \cdots, X_{r} \in \mathfrak{g}$ and $\nu \in \mathfrak{M}$. If $Y_{1}, \cdots, Y_{n}$ is a base for $g_{0}$ over $R$, it would be enough to prove that if

$$
\sum_{j=0}^{r} \sum_{1 \leqq i_{1}, \cdots, i_{j} \leqq n} c_{i_{1}, i_{2} \cdots i_{j}} Y_{i_{1}} Y_{i_{2}} \cdots Y_{i_{j}}=0 \quad\left(c_{i_{1} \cdots i_{j}} \in C\right)
$$

then

$$
\sum_{j=0}^{r} \sum_{1 \leqq i_{1}, \cdots, i_{j} \leqq n} c_{i_{1} i_{2} \cdots i_{j}} \gamma_{m}\left(Y_{i_{1}}, \cdots, Y_{i_{j}} ; \nu\right)=0 .
$$


Now $\phi$ and $\Phi$ being as above, we have seen that

$$
\Phi\left(x, Y_{i_{1}} \cdots Y_{i_{j}} ; m, \nu\right)=\phi\left(x m x^{-1}, b^{x}\right) \quad(x \in G)
$$

where

$$
b=\gamma_{m}\left(Y_{i_{1}}, Y_{i_{2}}, \cdots, Y_{i_{j}} ; \nu\right) .
$$

Hence we conclude that

$$
0=\sum_{j=0}^{r} \sum_{1 \leq i_{1}, \cdots, i_{j} \leqq n} c_{i_{1} \cdots i_{j}} \Phi\left(x, Y_{i_{1}} \cdots Y_{i_{j}} ; m, \nu\right)=\phi\left(x m x^{-1}, a^{x}\right)
$$

where

$$
a=\sum_{j=0}^{r} \sum_{1 \leqq i_{1}, \cdots, i_{j} \leqq m} c_{i_{1} \cdots i_{j}} \gamma_{m}\left(Y_{i_{1}}, \cdots, Y_{i} ; \nu\right) .
$$

Putting $x=1$, we get $\phi(m, a)=0$. This being true for every $\phi \in C^{\infty}(G)$, it follows from Lemma 13 that $a=0$.

Now we come to the second statement of the lemma. Put

$$
A(m)=\left(\operatorname{Ad}\left(m^{-1}\right)-I\right)_{q}
$$

and suppose $m \in \in^{\prime} M$. Let $\mathfrak{B}_{\mathrm{s}}$ denote the space of all elements in $\mathfrak{B}$ of degree $\leqq s$ and put $\mathfrak{B}_{-1}=\{0\}$. Then $\mathfrak{B}_{2} \bmod \mathfrak{B}_{\mathfrak{s - 1}}(s \geqq 0)$ is spanned by elements of the form $X_{1} X_{2} \cdots X_{2}$ where $X_{1}, \cdots, X_{2} \in \mathfrak{g}$. Moreover if $\left(i_{1}, i_{2}, \cdots, i_{s}\right)$ is any permutation of $(1,2, \cdots, s)$,

$$
X_{1} X_{2} \cdots X_{s}-X_{i_{1}} X_{i_{2}} \cdots X_{i_{s}} \in \mathfrak{B}_{s-1}
$$

(see [6(a)]). Let $\mathfrak{Q}_{d}$ and $\mathfrak{M}_{d}$ be the sets of homogeneous elements of degree $d$ in $\mathfrak{Q}$ and $\mathfrak{M}$ respectively. We shall prove by induction on $s$ that

$$
\sum_{d+e \leqq} \Gamma_{m}\left(\mathfrak{Q}_{d} \times \mathfrak{M}_{e}\right)=\mathfrak{B}_{\bullet} .
$$

If $Y_{1}, \cdots, Y_{d} \in q$ and $X_{1}, \cdots, X_{e} \in \mathfrak{m}$,

$$
\begin{aligned}
\Gamma_{m}\left(Y_{1} \cdots Y_{d} \times X_{1} \cdots X_{e}\right) \\
\quad=\left(L_{\mathrm{Ad}\left(m^{-1}\right) Y_{1}}-R_{Y_{1}}\right) \cdots\left(L_{\mathrm{Ad}\left(m^{-1}\right) Y_{d}}-R_{Y_{d}}\right)\left(X \cdots X_{\bullet}\right) .
\end{aligned}
$$

Put $D_{X}=L_{X}-R_{X}(X \in \mathfrak{g})$. Then since $D_{X} \mathfrak{B}_{r} \subset \mathfrak{B}_{r}(r \geqq 0)$, it is clear that

$$
\begin{aligned}
\Gamma_{m}\left(Y_{1} \cdots Y_{d}\right. & \left.\times X_{1} \cdots X_{e}\right) \\
& =\left(L_{\mathbf{A}(m) Y_{1}}+D_{Y_{1}}\right) \cdots\left(L_{\mathbf{A}(m) Y_{d}}+D_{Y_{d}}\right)\left(X_{1} \cdots X_{\odot}\right) \\
& \equiv\left(A(m) Y_{1}\right) \cdots\left(A(m) Y_{d}\right) X_{1} \cdots X_{e} \bmod \mathscr{B}_{d+e-1} .
\end{aligned}
$$

This shows that $\Gamma_{m}\left(\mathfrak{Q}_{d} \times \mathfrak{M}_{\odot}\right) \subset \mathfrak{B}_{s}$ if $d+e \leqq s$. Conversely since $m \in \in^{\prime} M, A(m)$ is nonsingular and so every element in $q$ can be written in the form $A(m) Y$ $(Y \in q)$. But elements of the form $Z_{1} \cdots Z_{d} X_{1} X_{2} \cdots X_{c}\left(Z_{1}, \cdots, Z_{d} \in q\right.$, 
$\left.X_{1}, \cdots, X_{e} \in \mathfrak{m}\right)$ with $d+e=s$ span $\mathfrak{B}_{8} \bmod \mathfrak{B}_{\mathfrak{s}-1}$ and the order of the factors is immaterial $\bmod \mathfrak{B}_{,-1}$. Therefore

$$
\sum_{d+=-s} \Gamma_{m}\left(\mathfrak{Q}_{d} \times \mathfrak{M}_{\epsilon}\right)+\mathfrak{B}_{s-1} \supset \mathfrak{B}_{s}
$$

and so our assertion followed by induction hypothesis. Moreover since $\mathfrak{g}$ is the direct sum of $\mathfrak{q}$ and $\mathfrak{m}, \mathfrak{B}_{\mathrm{s}}$ and $\sum_{d+e \leqq s} \mathfrak{Q}_{d} \times \mathfrak{M}_{e}$ have the same dimension (see Lemma 12 of $[6(c)]$ ). Therefore $\Gamma_{m}$ must be one-one.

Corollary. If $\nu_{1}, \nu_{2} \in \mathfrak{M}$, then $\Gamma_{m}\left(\nu_{1} \times \nu_{2}\right) \in \mathfrak{M}(m \in M)$ and

$$
\Gamma_{m}\left(b \nu_{1} \times \nu_{2}\right)=\Gamma_{m}\left(b \times \Gamma_{m}\left(\nu_{1} \times \nu_{2}\right)\right)
$$

It would be enough to consider the case when $\nu_{1}=X_{1} X_{2} \cdots X_{d}$ and $b=Z_{1} Z_{2} \cdots Z_{r}$ where $X_{1} \cdots X_{d} \in \mathfrak{m}$ and $Z_{1}, \cdots, Z_{r} \in \mathfrak{g}$. But then our assertions follow directly from the definition of $\Gamma_{m}$.

Let $f$ be a mapping of an analytic manifold $V$ into a vector space $U$. We say that $f$ is analytic if for every linear function $\mu$ on $U$ the function $p \rightarrow \mu(f(p))(p \in V)$ is analytic on $V$. Put $d_{+}(m)=\operatorname{det}\left(\operatorname{Ad}\left(m^{-1}\right)-I\right)_{\mathrm{q}}(m \in M)$. (In case $q=\{0\}$, we have to put $d_{+}(m)=1$.)

LEMMA 16. For each $b \in \mathfrak{B}$ there exists a nonnegative integer $r$ and an analytic mapping $\gamma_{b}$ of $M$ into $\mathfrak{Q} \times \mathfrak{M}$ such that

$$
\Gamma_{m}\left(\gamma_{b}(m)\right)=\left(d_{+}(m)\right)^{r} b \quad(m \in M) .
$$

Moreover if the degree of $b$ is $\leqq s$, the total degree of $\gamma_{b}(m)$ is also $\leqq s$.

We shall use induction on $s$. It is obviously sufficient to consider the case when

$$
b=Y_{1} Y_{2} \cdots Y_{d} X_{1} X_{2} \cdots X_{e}
$$

where $Y_{1}, \cdots, Y_{d} \in \mathfrak{q}, X_{1}, \cdots, X_{\bullet} \in \mathfrak{m}$ and $d+e=s$. Let $P(t, m)$ denote the characteristic polynomial of $A(m)=\left(\operatorname{Ad}\left(m^{-1}\right)-I\right)_{\mathfrak{q}}$ in the indeterminate $t$. Then if $q=\operatorname{dim} \mathfrak{q}$,

$$
P(t, m)=t^{q}-\sigma_{1}(m) t^{q-1}+\cdots+(-1)^{q} \sigma_{q}(m)
$$

where $\sigma_{1}, \cdots, \sigma_{q}$ are analytic functions on $M$ and $\sigma_{q}(m)=d_{+}(m)$. Put

$$
B(m)=(-1)^{q+1}\left\{(A(m))^{q-1}-\sigma_{1}(m)(A(m))^{q-2}+\cdots+(-1)^{q-1} \sigma_{q-1}(m) I_{\mathrm{q}}\right\}
$$

where $I_{\mathrm{q}}$ is the identity mapping of $\mathfrak{q}$. Then $B(m) A(m)=d_{+}(m) I_{\mathrm{q}}$. Put $Z_{i}=B(m) Y_{i}$ so that $A(m) Z_{i}=d_{+}(m) Y_{i}(i=1, \cdots, d)$. Also let

$$
Q(m)=(d !)^{-1} \sum Z_{i_{1}} Z_{i_{2}} \cdots Z_{i}
$$

where the sum extends over the permutations $\left(i_{1}, \cdots, i_{d}\right)$ of $(1,2, \cdots, d)$. Then it is clear that 


$$
\Gamma_{m}\left(Q(m) \times X_{1} \cdots X_{\epsilon}\right) \equiv\left(d_{+}(m)\right)^{d} Y_{1} Y_{2} \cdots Y_{d} X_{1} \cdots X_{\bullet} \bmod \mathbb{B}_{\ell-1} .
$$

Hence

$$
b_{0}(m)=\left(d_{+}(m)\right)^{d} b-\Gamma_{m}\left(Q(m) \times X_{1} \cdots X_{\bullet}\right) \in \mathfrak{B}_{\Omega_{-1}}
$$

and in view of the definition of $\Gamma_{m}$ it is obvious that $m \rightarrow b_{0}(m)$ is an analytic mapping of $M$ into $\mathfrak{B}_{s-1}$. Therefore if $b_{1}, \cdots, b_{N}$ is a base for $\mathfrak{B}_{s_{-1}}$,

$$
b_{0}(m)=\sum_{i=1}^{N} a_{i}(m) b_{i}
$$

where $a_{i}(m)$ are analytic functions on $M$. Now by induction hypothesis we can choose integers $r_{i} \geqq 0$ and analytic mappings $\gamma_{b_{i}}$ of $M$ into $\mathfrak{Q} \times \mathfrak{M}$ such that

$$
\Gamma_{m}\left(\gamma_{b_{i}}(m)\right)=\left(d_{+}(m)\right)^{r_{i} b_{i}} \quad(i=1, \cdots, N)
$$

and the total degree of $\gamma_{b_{i}}(m)$ is $\leqq s-1(m \in M)$. Put $r=\max _{1 \leqq i \leqq N}\left(d, r_{i}\right)$ and

$$
\gamma_{b}(m)=\left(d_{+}(m)\right)^{r-d} Q(m) \times X_{1} \cdots X_{\theta}+\sum_{i=1}^{N} a_{i}(m)\left(d_{+}(m)\right)^{r-r_{i}} \gamma_{b_{i}}(m) .
$$

Then $\gamma_{b}$ is an analytic mapping of $M$ into $\mathfrak{B}$,

$$
\Gamma_{m}\left(\gamma_{b}(m)\right)=\left(d_{+}(m)\right)^{r} b
$$

and the total degree of $\gamma_{b}(m)$ is $\leqq s(m \in M)$. This proves the lemma.

Now put $\mathfrak{Q}^{\prime}=\sum_{d \geqq 1} \mathfrak{Q}_{d}$ and let $m \in \mathcal{C}^{\prime} M$. Then if $b \in \mathfrak{B}$, it follows from Lemma 15 that there is a unique element $\delta_{m}(b) \in \mathfrak{M}$ such that

$$
b-\delta_{m}(b)=b-\Gamma_{m}\left(1 \times \delta_{m}(b)\right) \in \Gamma_{m}\left(\mathfrak{Q}^{\prime} \times \mathfrak{M}\right) \text {. }
$$

Moreover in view of Lemma $16, m \rightarrow \delta_{m}(b)$ is an analytic mapping of ' $M$ into $\mathfrak{M}$. Therefore it is obvious that there exists a (unique) analytic differential operator $\delta(b)$ on ' $M$ such that $\delta_{m}(b)$ is its local expression at any point $m \in{ }^{\prime} M$.

Lemma 17. If $a \in \mathfrak{B}$ and $m \in M$,

$$
(\delta(a))^{m}=\delta\left(a^{m}\right) \text {. }
$$

For any $m \in M$, the mappings $q \rightarrow q^{m}$ and $\nu \rightarrow \nu^{m}(q \in \mathfrak{Q}, \nu \in \mathfrak{M})$ are linear isomorphisms of $\mathfrak{Q}$ and $\mathfrak{M}$ respectively onto themselves. Hence there exists a linear isomorphism of $\mathfrak{Q} \times \mathfrak{M}$ onto itself which maps $q \times \nu$ onto $q^{m} \times \nu^{m}$. We denote it again by Ad $(m)$. On the other hand it is obvious from the definition of $\Gamma_{m_{1}}\left(m_{1} \in M\right)$ that

$$
\operatorname{Ad}(m)\left(\Gamma_{m_{1}}(a \times \nu)\right)=\Gamma_{m m_{1} m^{-1}}\left(a^{m} \times \nu^{m}\right)
$$

for $a \in \mathfrak{B}$ and $\nu \in \mathfrak{M}$. So in particular if $m_{1} \in \in^{\prime} M$, 


$$
a-\Gamma_{m_{1}}\left(1 \times \delta_{m_{1}}(a)\right) \in \Gamma_{m_{1}}\left(\mathfrak{Q}^{\prime} \times \mathfrak{M}\right)
$$

and therefore

$$
a^{m}-\operatorname{Ad}(m)\left(\Gamma_{m_{1}}\left(1 \times \delta_{m_{1}}(a)\right)\right) \in \Gamma_{m m_{1} m^{-1}}\left(\mathfrak{Q}^{\prime} \times \mathfrak{M}\right)
$$

since $\operatorname{Ad}(m)\left(\mathfrak{Q}^{\prime} \times \mathfrak{M}\right)=\mathfrak{Q}^{\prime} \times \mathfrak{M}$. This shows that

$$
\delta_{m m_{1} m^{-1}}\left(a^{m}\right)=\operatorname{Ad}(m)\left(\delta_{m_{1}}(a)\right)
$$

and therefore it follows from Lemma 14 that $(\delta(a))^{m}=\delta\left(a^{m}\right)$.

CoRollary. If $a$ is an element in $\mathfrak{B}$ such that $a^{m}=a$ for all $m \in M$, then $\delta(a)$ is an invariant differential operator on ' $M$.

This is an immediate consequence of Lemma 17.

6. The relationship between invariants of the Weyl group and certain differential operators. Let $z$ be an element in the center of $\mathfrak{B}$. We shall now try to determine the differential operator $\delta(z)$ on ' $M$. Let us first recall a few facts about reductive Lie algebras (see Koszul [9]). Let $\mathfrak{l}$ be a reductive Lie algebra over $C$ and let $\mathfrak{c}$ and $\mathfrak{l}^{\prime}$ respectively denote the center and the derived algebra of $\mathfrak{l}$. Then $\mathfrak{l}^{\prime}$ is semisimple and if $\Gamma$ is a Cartan subalgebra of $\mathfrak{l}$, it is obvious that $\Gamma=\mathfrak{c}+\Gamma^{\prime}$ where $\Gamma^{\prime}=\Gamma \cap \mathfrak{l}^{\prime}$ is a Cartan subalgebra of $\mathfrak{l}^{\prime}$. By a root of $\mathfrak{l}$ (with respect to $\Gamma$ ) we mean a root $\alpha$ of $\mathfrak{l}^{\prime}$ (with respect to $\Gamma^{\prime}$ ). We extend $\alpha$ to a linear function on $\Gamma$ by setting $\alpha(H)=0(H \in \mathfrak{c})$. Let $\mathfrak{w}$ be the Weyl group of $\mathfrak{l}^{\prime}$ with respect to $\Gamma^{\prime}$. If $s \in \mathfrak{m}$ we also extend $s$ to a linear transformation on $\Gamma$ by setting $s H=H(H \in \mathfrak{c})$. For every root $\alpha$ choose an element $X_{\alpha} \neq 0$ such that $\left[H, X_{\alpha}\right]=\alpha(H) X_{\alpha}$ for all $H \in \Gamma$. Introduce some lexicrographic order among roots and let $\alpha_{1}, \cdots, \alpha_{r}$ be all the (distinct) positive roots under this order. Denote by $\mathbb{R}$ the universal enveloping algebra of $\mathfrak{l}$ and by $\mathfrak{U}$ the subalgebra of $\mathfrak{R}$ generated by $(1, \Gamma)$. Let $u \rightarrow u^{s}(u \in \mathfrak{U})$ denote the automorphism of $\mathfrak{U}$ which coincides with $s$ on $\Gamma(s \in \mathfrak{w})$.

Lemma 18. Let $\mathbb{Z}$ be the center of $\mathbb{R}$. Then for every $z \in \mathbb{Z}$ there exists a unique element $\gamma^{\prime}(z) \in \mathfrak{U}$ such that $z-\gamma^{\prime}(z) \in \sum_{i=1}^{r} \& X_{\alpha_{i}}$. Furthermore $z \rightarrow \gamma^{\prime}(z)(z \in Z)$ is an algebraic isomorphism of $\mathbb{3}$ into $\mathfrak{u}$.

If $c=\{0\}$ this is the same as Lemma 36 of $[6(b)]$. The proof in the general case also is practically the same and so we shall not repeat it here.

Let us say that an element $u \in \mathfrak{U}$ is an invariant (of $\mathfrak{w}$ ) if $u^{s}=u$ for all $s \in \mathfrak{w}$. Let $J$ denote the subalgebra of $\mathfrak{U}$ consisting of all invariants. Put $\rho=\left(\alpha_{1}+\cdots+\alpha_{r}\right) / 2$ and let $\lambda$ denote the automorphism of $\mathfrak{U}$ given by $\lambda(H)=H-\rho(H)(H \in \Gamma)$. Define $\gamma(z)=\lambda\left(\gamma^{\prime}(z)\right)(z \in ß)$.

Lemma 19. $z \rightarrow \gamma(z)(z \in Z)$ is an algebraic isomorphism of 8 onto $J$.

If $c=\{0\}$ this follows from the results of [6(b), Part II, see equation (13) p. 71 and Lemmas 38 and 39]. In the general case let $\mathfrak{S}, \mathfrak{l}^{\prime}$ and $\mathfrak{u}^{\prime}$ be the sub- 
algebras of $\mathfrak{R}$ generated by $(1, \mathfrak{c}),\left(1, \mathfrak{l}^{\prime}\right)$ and $\left(1, \Gamma^{\prime}\right)$ respectively. Then $\mathfrak{l}=\mathfrak{l}^{\prime} \mathfrak{C}$, $\mathfrak{Z}=\mathfrak{Z}^{\prime} \mathfrak{E}$ and $J=J^{\prime} \mathfrak{E}$ where $\mathfrak{Z}^{\prime}$ is the center of $\mathfrak{R}^{\prime}$ and $J^{\prime}=J \cap \mathfrak{u}^{\prime}$. Since $\gamma(c)=c(c \in \mathfrak{C})$ our assertion now follows from the corresponding result for $\mathfrak{l}^{\prime}$.

For every automorphism $\tau$ of $\mathfrak{l}$ let $b \rightarrow b^{\tau}(b \in \mathfrak{R})$ denote the corresponding automorphism of $\mathfrak{R}$.

LemMa 20. The mat ping $\gamma$ defined above is independent of the lexicographic order of roots used in its definition. If $\tau$ is any automorphism of $\mathfrak{l}$ which leaves $\Gamma$ invariant, then $\gamma\left(z^{\tau}\right)=(\gamma(z))^{\tau}(z \in \mathbb{Z})$. Finally let $b \rightarrow b^{*}(b \in \mathfrak{R})$ denote the antiautomorphism of $\mathfrak{R}$ which maps $X$ on $-X(X \in \mathfrak{l})$. Then $\gamma\left(z^{*}\right)=(\gamma(z))^{*}(z \in \mathbb{Z})$.

Let $\beta$ be a linear function on $\Gamma$ and $\tau$ an automorphism of $\mathfrak{l}$ such that $\tau \Gamma=\Gamma$. Then we denote by $\tau \beta$ the function $H \rightarrow \beta\left(\tau^{-1} H\right)(H \in \Gamma)$. If $\alpha$ is a root, it is obvious that $\tau \alpha$ is also a root and $\tau X_{\alpha} \in C X_{\tau \alpha}$. Now first suppose $\tau$ permutes the positive roots $\left(\alpha_{1}, \cdots, \alpha_{r}\right)$ among themselves. Then $\tau \rho=\rho$ and if $\nu$ is the automorphism of $\mathfrak{u}$ given by $\nu(H)=H+\rho(H)(H \in \Gamma), \nu$ commutes with $\tau$ (if the latter is regarded as an automorphism of $\mathfrak{u}$ ). Now let $z \in 3$. Then $z-\nu(\gamma(z)) \in \sum_{i=1}^{r} \mathfrak{\&} X_{\alpha_{i}}$ from the definition of $\gamma$. Hence

$$
z^{\tau}-(\nu(\gamma(z)))^{\tau}=z^{\tau}=\nu\left((\gamma(z))^{\tau}\right) \in \sum_{i=1}^{r} \mathfrak{l}_{\tau} X_{\alpha_{i}}=\sum_{i=1}^{r} \mathfrak{l} X_{\alpha_{i}}
$$

Since $z^{\tau} \in 3$, it follows from Lemma 19 that $\nu\left(\gamma\left(z^{\tau}\right)\right)=\nu\left((\gamma(z))^{\tau}\right)$ and therefore $\gamma\left(z^{\tau}\right)=(\gamma(z))^{\tau}$. Now suppose $\tau$ is any automorphism of $\mathfrak{l}$ leaving $\Gamma$ invariant. Then $\left(\tau \alpha_{1}, \cdots, \tau \alpha_{r}\right)$ is the set of positive roots in a suitable lexicographic order and hence there exists $\left({ }^{7}\right)$ an element $s \in \mathfrak{w}$ such that $\left(s \alpha_{1}, \cdots, s \alpha_{r}\right)$ and $\left(\tau \alpha_{1}, \cdots, \tau \alpha_{r}\right)$ are the same apart from order. As is well known $s$ can be extended to an "inner" automorphism( $\left(^{8}\right)$ of $\mathfrak{l}$. Then $z^{z}=z(z \in \mathfrak{Z})$ and $\tau^{\prime}=\tau s^{-1}$ permutes $\left(\alpha_{1}, \cdots, \alpha_{r}\right)$ among themselves. But then $z^{r}=z^{\tau^{\prime}}$ and $(\gamma(z))^{r}$ $=(\gamma(z))^{r^{\prime}}$ since $(\gamma(z))^{\circ}=\gamma(z)(z \in Z)$. Hence in view of the above proof,

$$
\gamma\left(z^{\tau}\right)=\gamma\left(z^{\tau^{\prime}}\right)=(\gamma(z))^{\tau^{\prime}}=(\gamma(z))^{\tau} .
$$

Now put $\nu_{\tau}=\tau \nu \tau^{-1}(\tau$ being regarded as an automorphism of $\mathfrak{U})$. Then $\nu_{\tau}(H)$ $=H+\tau \rho(H)(H \in \Gamma)$ and $\nu_{r}\left(\gamma\left(z^{r}\right)\right)=(\nu(\gamma(z)))^{\tau}$. This shows that

$$
z^{\tau}-\nu_{\tau}\left(\gamma\left(z^{\tau}\right)\right)=(z-\nu(\gamma(z)))^{\tau} \in \sum_{i=1}^{r} \mathfrak{R} X_{\tau \alpha_{i}} \cdot
$$

Replacing $z$ by $z^{r^{-1}}$, we get

$\left.{ }^{7}\right)$ This follows from the fact that one fundamental system of roots can be transformed into every other (apart from order) by an element of $\mathfrak{m}$ (see Weyl [14(b)] and [6(f), Corollary 2 to Lemma 4]).

(8) This means that there exists a transformation of the adjoint group of $\mathfrak{l}$ which coincides with $s$ on $\Gamma$ (see Weyl [14(b)]). 


$$
z-\nu_{r}(\gamma(z)) \in \sum_{i=1}^{r} \& X_{r \alpha_{i}}
$$

Now $\left(\alpha_{1}^{\prime}, \cdots, \alpha_{r}^{\prime}\right)$ being the set of all positive roots in any other order, put $\rho^{\prime}=\left(\alpha_{1}^{\prime}+\cdots+\alpha_{r}^{\prime}\right) / 2$ and let $\nu^{\prime}$ be the automorphism of $\mathfrak{u}$ given by $\nu^{\prime}(H)=H+\rho^{\prime}(H)(H \in \Gamma)$. Then in order to prove the first statement of the lemma, we have to show that

$$
z-\nu^{\prime}(\gamma(z)) \in \sum_{i=1}^{r} \& X_{\alpha_{i}^{\prime}}^{\prime}
$$

But, as has already been mentioned above, we can choose $\left({ }^{7}\right)$ an element $s \in \mathfrak{m}$ such that the two sets $\left(\alpha_{1}^{\prime}, \cdots, \alpha_{r}^{\prime}\right)$ and $\left(s \alpha_{1}, \cdots, s \alpha_{r}\right)$ are the same. Then $\rho^{\prime}=s \rho$ and therefore $\nu^{\prime}=\nu_{s}$ and

$$
z-\nu^{\prime}(\gamma(z))=z-\nu_{s}(\gamma(z)) \in \sum_{i=1}^{r} \mathfrak{R} X_{s \alpha_{i}}=\sum_{i=1}^{r} \mathfrak{l} X_{\alpha_{i}^{\prime}}
$$

Now we come to the last part. One proves without difficulty by using the notion of rank (see $[6(b)$, p. 79]) that actually

$$
z-\nu(\gamma(z)) \in \sum_{1 \leqq i, j \leqq r} X_{-\alpha_{i}} \& X_{\alpha_{j}} \quad(z \in 3) .
$$

Hence

$$
z^{*}-(\nu(\gamma(z)))^{*} \in \sum_{1 \leqq i, j \leqq r} X_{\alpha_{j}} \& X_{-\alpha_{i}} .
$$

Define the automorphism $\nu^{*}$ of $\mathfrak{U}$ by $\nu^{*}(u)=\left(\nu\left(u^{*}\right)\right)^{*}(u \in \mathfrak{U})$. Then $\nu^{*}(H)$ $=H-\rho(H)(H \in \Gamma)$ and if we replace $z$ by $z^{*}$ in the above equation, we have

$$
z-\nu^{*}\left(\left(\gamma\left(z^{*}\right)\right)^{*}\right) \in \sum_{1 \leqq i, j \leqq r} X_{\alpha_{j}} \& X_{-\alpha_{i}} \text {. }
$$

On the other hand it is obvious that under a suitable order $\left(-\alpha_{1}, \cdots,-\alpha_{r}\right)$ is the set of all positive roots. Since $-\left(\alpha_{1}+\cdots+\alpha_{r}\right) / 2=-\rho$, it follows from what has been proved above that

$$
z-\nu^{*}(\gamma(z)) \in \sum_{i=1}^{r} \mathfrak{R} X_{-\alpha_{i}} .
$$

This shows that $\gamma(z)=\left(\gamma\left(z^{*}\right)\right) *$ and therefore $\gamma\left(z^{*}\right)=(\gamma(z))^{*}(z \in Z)$.

COROLlaRY. If $\tau$ is any anti-automorphism of $\mathfrak{R}$ such that $\mathfrak{l}^{\tau}=\mathfrak{l}$ and $\Gamma^{\tau}=\Gamma$, then $\gamma\left(z^{\tau}\right)=(\gamma(z))^{r}(z \in \mathbb{B})$.

The mapping $b \rightarrow\left(b^{\tau}\right)^{*}$ is obviously an automorphism of $\mathfrak{l}$ which leaves $\mathfrak{l}$ and $\Gamma$ invariant. Hence the corollary follows from the above lemma.

We shall now apply Lemma 19 to our problem. Let 3 and $\not_{M}$ denote the centers of $\mathfrak{B}$ and $\mathfrak{M}$ respectively and $\mathfrak{U}$ the subalgebra of $\mathfrak{B}$ generated by 
$(1, \mathfrak{h})$. Let $W$ be the Weyl group of $g$ (with respect to $\mathfrak{h}$ ) and $W_{-}$the subgroup generated by the Weyl reflexions $s_{\alpha}$ corresponding to $\alpha \in P_{-}$. Also denote by $J$ and $J_{-}$respectively the subalgebras of $\mathfrak{U}$ consisting of all invariants of $W$ and $W_{\text {. }}$. It is obvious that $\mathfrak{m}$ is reductive and $\mathfrak{h}$ is a Cartan subalgebra of $m$. Then in accordance with Lemma 19 we have the isomorphisms $z \rightarrow \gamma(z)(z \in \mathbb{Z})$ and $w \rightarrow \gamma_{-}(w)\left(w \in Z_{M}\right)$ of $\mathbb{Z}$ and $Z_{M}$ onto $J$ and $J_{-}$respectively. Since $J \subset J_{-}$, there exists an isomorphism $z \rightarrow \mu(z)$ of $\mathbb{Z}$ into $\mathfrak{Z}_{M}$ such that $\gamma(z)=\gamma_{-}(\mu(z))$. The significance of $\mu$ is expressed by the theorem below. First let us recall that $d_{+}(m)=\operatorname{det}\left(\operatorname{Ad}\left(m^{-1}\right)-I\right)_{q} \neq 0$ if $m \in \in^{\prime} M$ and therefore $\left|d_{+}\right|^{1 / 2}$ and $\left|d_{+}\right|^{-1 / 2}$ are analytic functions on ' $M$. Moreover $\mu(z)$, being an element of $\mathfrak{M}$, is a differential operator on $M$.

THEOREM 1. If $z \in B$ and $\tau$ is an invariant distribution on ' $M$, then

$$
\delta(z) \tau=\left|d_{+}\right|^{-1 / 2} \mu(z)\left(\left|d_{+}\right|^{1 / 2} \tau\right) .
$$

Let $D=\left|d_{+}\right|^{-1 / 2} \mu(z) \circ\left|d_{+}\right|^{1 / 2}$ where $\circ$ denotes the operator product. It is clear from Lemma 7 that $\left|d_{+}\right|^{-1 / 2}, \mu(z),\left|d_{+}\right|^{1 / 2}$ are all invariant differential operators on ' $M$. Therefore $D$ is also invariant. Also $\delta(z)$ is invariant from the corollary to Lemma 17. Put $\Theta=\delta(z)-D$. We have to show that $\tau\left(\Theta^{*} f\right)=0$ for any $f \in C_{c}^{\infty}(' M)$. Let $M^{*}$ be the analytic subgroup of the adjoint group of $\mathfrak{g}_{0}$ corresponding to $\mathfrak{m}_{\mathfrak{t}_{0}}$. Then $M^{*}$ is compact. Put $g^{m^{*}}=g^{m}\left(g \in C_{c}^{\infty}\left({ }^{\prime} M\right)\right.$, $m^{*} \in M^{*}$ ) where $m$ is any element in $\bar{M}$ (see Lemma 7 for notation) whose image in $M^{*}$ is $m^{*}$. Moreover normalize the Haar measure $d m^{*}$ on $M^{*}$ in such a way that $\int_{M^{*}} d m^{*}=1$. Then since $\tau$ and $\Theta^{*}$ are both invariant, it is clear that

$$
\tau\left(\Theta^{*} f\right)=\tau\left(\left(\Theta^{*} f\right)^{m^{*}}\right)=\tau\left(\Theta^{*}\left(f^{m^{*}}\right)\right)=\tau\left(\Theta^{*} \bar{f}\right)
$$

where

$$
\bar{f}(m)=\int_{M^{*}} f^{m^{*}}(m) d m^{*} \quad\left(m \in \in^{\prime} M\right) .
$$

Since $\bar{f} \in I_{c}^{\infty}\left({ }^{\prime} M\right)$, it would be enough to prove that $\Theta^{*} g=0$ for every $g \in I_{c}^{\infty}\left({ }^{\prime} M\right)$. On the other hand if $d m$ is the Haar measure of $M$ and $f \in C_{c}^{\infty}\left({ }^{\prime} M\right)$, $g \in I_{c}^{\infty}(' M)$, it is evident that

$$
\int f \Theta^{*} g d m=\int \bar{f} \Theta^{*} g d m=\int(\Theta \bar{f}) g d m
$$

where $\bar{f}$ is defined as above. Hence it is sufficient to prove the following lemma.

Lemma 21. If $f \in I_{c}^{\infty}\left({ }^{\prime} M\right), \Theta f=0$.

$G^{\prime}$ being the set of regular elements in $G$, put $A^{\prime}=A \cap G^{\prime}$. Since $\Theta f$ 
$\in I_{c}^{\infty}(' M)$ it would obviously be enough (see Lemmas 7 and 8 ) to show that $f(h, \Theta)=0$ for $h \in A^{\prime}$. For this we need some additional results which will be obtained in the next two sections.

7. Differential operators on $A^{\prime}$. Put $8=\sum_{\alpha \in P}\left(C X_{\alpha}+C X_{-\alpha}\right)$ and $z_{-}=\sum_{\alpha \in P_{-}}\left(C X_{\alpha}+C X_{-\alpha}\right)$. Then $\mathfrak{g}=\mathfrak{z}+\mathfrak{h}, \mathfrak{m}=z_{-}+\mathfrak{h}$ and $z=\mathfrak{q}+z_{-}$where all sums are direct. Let $S(B)$ and $S\left(z_{-}\right)$denote the symmetric algebras over 8 and $\mathbb{B}_{\text {- respectively and }} \mathfrak{S}$ and $\mathfrak{S}_{-}$their images in $\mathfrak{B}$ under the canonical mapping of $S(\mathfrak{g})$ onto $\mathfrak{B}$. Let $\mathfrak{S}_{r}$ be the space of homogeneous elements of degree $r$ in $\mathfrak{S}$. Put $\mathfrak{S}^{\prime}=\sum_{r \geqq 1} \mathfrak{S}_{r}$ and $\mathfrak{S}_{-}^{\prime}=\mathfrak{S}_{-} \cap \mathfrak{S}^{\prime}$. $\mathfrak{U}$ being the subalgebra of $\mathfrak{B}$ generated by $(1, \mathfrak{h})$, it is obvious that $\mathfrak{B}=\subseteq \mathfrak{U}$ and $\mathfrak{M}=\mathfrak{S}_{-} \mathfrak{U}$ (see [6(c), Lemma 12]). Let $\Gamma_{m}(m \in M)$ denote the mapping of Lemma 15. Notice that $A^{\prime} C^{\prime} M$ and $\mathfrak{u} \subset \mathfrak{M}$.

Lemma 22. If $h \in A^{\prime}, \Gamma_{h}$ defines a one-one mapping $\mathfrak{S} \times \mathfrak{u}$ onto $\mathfrak{B}$ and also a one-one mapping of $\mathfrak{S}_{-} \times \mathfrak{u}$ onto $\mathfrak{M}$.

The proof of these statements is exactly parallel to that of Lemma 9.

We note that $\mathfrak{B g}=\mathfrak{S}^{\prime} \mathfrak{U}+\mathfrak{u h}$ and in view of the corollary to Lemma 15 ,

$$
\Gamma_{h}(\mathfrak{u h} \times \mathfrak{u})=\Gamma_{h}\left(\mathfrak{U} \times \Gamma_{h}(\mathfrak{h} \times \mathfrak{u})\right)=\{0\} \quad(h \in A)
$$

since $\Gamma_{h}(\mathfrak{h} \times \mathfrak{u})=\{0\}$. Hence applying the same corollary once again, we get

$$
\Gamma_{h}(\mathfrak{B g} \times \mathfrak{u})=\Gamma_{h}\left(\mathfrak{S}^{\prime} \mathfrak{u} \times \mathfrak{u}\right) \subset \Gamma_{\mathfrak{h}}\left(\mathbb{S}^{\prime} \times \mathfrak{u}\right) .
$$

This proves that

$$
\Gamma_{h}\left(\mathfrak{S}^{\prime} \times \mathfrak{u}\right)=\Gamma_{h}\left(\mathfrak{B}^{\prime} \times \mathfrak{u}\right)
$$

where $\mathfrak{B}^{\prime}=\mathfrak{B g}$. Similarly $\Gamma_{h}\left(\mathfrak{S}_{-}^{\prime} \times \mathfrak{u}\right)=\Gamma_{h}\left(\mathfrak{M}^{\prime} \times \mathfrak{U}\right)$ where $\mathfrak{M}^{\prime}=\mathfrak{M m}$.

For each root $\alpha$ let $\xi_{\alpha}$ be the character of $A$ given by $\operatorname{Ad}(h) X_{\alpha}=\xi_{\alpha}(h) X_{\alpha}$ $(h \in A)$ and consider the multiplicative group $0 \Xi$ generated by all $\xi_{\alpha}$. Also let ${ }_{0} \Xi_{-}$be the subgroup of ${ }_{0} \Xi$ generated by $\xi_{\alpha}\left(\alpha \in P_{-}\right)$. We denote by $\Xi$ the space of finite linear combinations of characters in $0 \Xi$ with coefficients in $C$. Obviously $\Xi$ is a ring under ordinary multiplication and the subspace $\Xi_{-}$spanned by $0 \Xi_{-}$is a subring of $\Xi$. Put

$$
d(h)=\operatorname{det}\left(\operatorname{Ad}\left(h^{-1}\right)-I\right)_{8}, \quad d_{-}(h)=\operatorname{det}\left(\operatorname{Ad}\left(h^{-1}\right)-I\right)_{8-}
$$

where the subscripts denote restrictions to the corresponding subspace.

Lemma 23. For each $b \in \mathfrak{B}$ we can choose elements $v_{1}, \cdots, v_{N} \in \mathfrak{B} \times \mathfrak{u}$, $\xi_{1}, \cdots, \xi_{N} \in \Xi$ and an integer $r \geqq 0$ such that

for all $h \in A$.

$$
\Gamma_{h}\left(\sum_{i=1}^{N} \xi_{i}(h) v_{i}\right)=(d(h))^{r} b
$$

Define $\mathfrak{B}_{\mathrm{s}}(s \geqq-1)$ as in the proof of Lemma 15 and suppose $b \in \mathfrak{B}_{\mathrm{s}}(s \geqq 0)$. We shall use induction on $s$. Let $X_{1}, \cdots, X_{n}$ be a base of $\mathfrak{g}$ consisting of the 
elements $X_{\alpha}, X_{-\alpha}(\alpha \in P)$ and a base of $\mathfrak{h}$. Then it is obviously sufficient to consider the case when $b=Y_{1} Y_{2} \cdots Y_{s}$ where each $Y_{i}$ is equal to some $X_{j}$. Since the order of the factors is immaterial $\bmod \mathfrak{B}_{s-1}$, we may assume that $Y_{j} \in \mathfrak{h}$ for $t<j \leqq s$ while $Y_{j} \notin \mathfrak{h}$ for $1 \leqq j \leqq t$. Also we may suppose that $t \geqq 1$ for otherwise if $t=0, b \in \mathfrak{U}$ and $\Gamma_{h}(1 \times b)=b(h \in A)$. Put $Y_{t+1} \cdots Y_{s}=u \in \mathfrak{U}$ $(u=1$ if $t=s)$ and let $\eta_{i}$ be the character of $A$ given by $\operatorname{Ad}(h) Y_{i}=\eta_{i}(h) Y_{i}$ $(1 \leqq i \leqq t)$. Then $\eta_{i}=\xi_{\alpha}$ for some root $\alpha$ and therefore $\eta_{i}(h) \neq 1$ if $h \in A^{\prime}$. For any $j(1 \leqq j \leqq t)$ put $y_{j}=Y_{j} \cdots Y_{t}$. Then $\left({ }^{9}\right)$

$$
\begin{aligned}
\Gamma_{h}\left(y_{j} \times u\right) & =\left(L_{\mathrm{Ad}\left(h^{-1}\right) Y_{j}}-R_{y_{j}}\right) \Gamma_{h}\left(y_{j+1} \times u\right) \\
& =\left(\operatorname{Ad}\left(h^{-1}\right) Y_{j}-Y_{j}\right) \Gamma_{h}\left(y_{j+1} \times u\right)+\left[Y_{j}, \Gamma_{h}\left(y_{j+1} \times u\right)\right] \\
& =\left(\eta_{j}\left(h^{-1}\right)-1\right) Y_{j} \Gamma_{h}\left(y_{j+1} \times u\right)+\left[Y_{j}, \Gamma_{h}\left(y_{j+1} \times u\right)\right] .
\end{aligned}
$$

Therefore it follows easily by induction on $t-j$ that for each $j$ we can choose elements $b_{i j} \in \mathscr{B}_{s+l-j-1}, \zeta_{i j} \in \Xi\left(1 \leqq i \leqq N_{j}\right)$ such that

$$
\Gamma_{h}\left(y_{j} \times u\right)=\prod_{j \leqq i \leqq t}\left(\eta_{i}\left(h^{-1}\right)-1\right) y_{j} u+\sum_{i=1}^{N_{j}} \zeta_{i j}(h) b_{i j} .
$$

Putting $j=1$ and $b_{i 1}=b_{i}, \zeta_{i 1}=\zeta_{i}, 1 \leqq i \leqq N_{1}$,

$$
\Gamma_{h}\left(Y_{1} \cdots Y_{t} \times u\right)=\prod_{i=1}^{t}\left(\eta_{i}\left(h^{-1}\right)-1\right) b+\sum_{i=1}^{N_{1}} \zeta_{i}(h) b_{i} .
$$

Also it is obviously possible to find an integer $k \geqq 0$ and $\zeta_{0} \in \Xi$ such that

$$
\zeta_{0}(h) \prod_{1 \leqq i \leqq t}\left(\eta_{i}\left(h^{-1}\right)-1\right)=(d(h))^{k} \quad(h \in A) .
$$

Hence if we put $v_{0}=\left(Y_{1} Y_{2} \cdots Y_{t}\right) \times u$,

$$
\Gamma_{h}\left(\zeta_{0}(h) v_{0}\right)=(d(h))^{k} b+\sum_{j=1}^{N_{1}} \zeta_{0}(h) \zeta_{j}(h) b_{j} .
$$

Since $\Xi$ is a ring and $b_{j} \in \mathfrak{B}_{s-1}\left(1 \leqq j \leqq N_{1}\right)$ our assertion now follows from the induction hypothesis.

If $b \in \mathfrak{B}$ and $h \in A^{\prime}$, we conclude from Lemma 22 that there exists a unique element $\beta_{h}(b) \in \mathfrak{U}$ such that

$$
b-\beta_{h}(b) \in \Gamma_{h}\left(\mathfrak{S}^{\prime} \times \mathfrak{u}\right)=\Gamma_{h}\left(\mathfrak{B}^{\prime} \times \mathfrak{u}\right) .
$$

Define $v_{i}, \xi_{i}$ and $r$ as in Lemma 23 and choose $u_{i} \in \mathfrak{U}$ such that $v_{i}-\left(1 \times u_{i}\right)$ $\in \mathfrak{B}^{\prime} \times \mathfrak{u}$. Then since $d(h)$ is never zero if $h \in A^{\prime}$, it is clear that

$$
\beta_{h}(b)=(d(h))^{-r} \sum_{i=1}^{N} \xi_{i}(h) u_{i} \quad\left(h \in A^{\prime}\right) .
$$

Thus we have obtained the following result.

(9) If $a_{1}, a_{2}$ are two elements of an associative algebra, we write $\left[a_{1}, a_{2}\right]=a_{1} a_{2}-a_{2} a_{1}$. 
Corollary. If $b \in \mathfrak{B}$, it is possible to choose elements $u_{1}, \cdots, u_{N} \in \mathfrak{U}$, $\xi_{1}, \cdots, \xi_{N} \in \in_{0} \Xi$ and an integer $r \geqq 0$ such that

$$
\beta_{h}(b)=(d(h))^{-r} \sum_{i=1}^{N} \xi_{i}(h) u_{i}
$$

for all $h \in A^{\prime}$.

Now suppose $b \in \mathfrak{M}$ and $h \in A^{\prime}$. Then from Lemma 22 we can choose $b_{0} \in \mathfrak{U}$ such that $b-b_{0} \in \Gamma_{h}\left(\Im_{-}^{\prime} \times \mathfrak{u}\right)$. But

$$
\Gamma_{h}\left(\Im_{\prime}^{\prime} \times \mathfrak{u}\right) \subset \Gamma_{h}\left(\mathfrak{B}^{\prime} \times \mathfrak{u}\right)
$$

and so, in view of the uniqueness of $\beta_{h}(b)$, we conclude that $b_{0}=\beta_{h}(b)$. Hence

$$
b-\beta_{h}(b) \in \Gamma_{h}\left(S_{-}^{\prime} \times \mathfrak{u}\right)=\Gamma_{h}\left(\mathfrak{M}^{\prime} \times \mathfrak{U}\right) .
$$

The following lemma and corollary are proved in the same way as Lemma 23.

Lemma 24. For each $b \in \mathfrak{M}$ we can choose elements $v_{1}, \cdots, v_{N} \in \mathfrak{M} \times \mathfrak{u}$, $\xi_{1}, \cdots, \xi_{N} \in \Xi_{-}$and an integer $r \geqq 0$ such that

$$
\Gamma_{h}\left(\sum_{i=1}^{N} \xi_{i}(h) v_{i}\right)=\left(d_{-}(h)\right)^{r} b
$$

for all $h \in A$.

Corollary. If $b \in \mathfrak{M}$ it is possible to select elements $u_{1}, \cdots, u_{N} \in \mathfrak{U}$, $\xi_{1}, \cdots, \xi_{N} \in \Theta_{0} \Xi_{-}$and an integer $r \geqq 0$ such that

$$
\beta_{h}(b)=\left(d_{-}(h)\right)^{-r} \sum_{i=1}^{N} \xi_{i}(h) u_{i}
$$

for all $h \in A^{\prime}$.

We now regard $A^{\prime}$ as an open submanifold of the Lie group $A$. It follows from the above results that for each $b \in \mathfrak{B}$ there exists an analytic differential operator $\beta(b)$ on $A^{\prime}$ whose local expression at $h \in A^{\prime}$ is $\beta_{h}(b)$.

Lemma 25. Suppose $b \in \mathfrak{M}$ and $f \in I^{\infty}(' M)$. Then if $h \in A^{\prime}$,

$$
f(h, b)=f\left(h, \beta_{h}(b)\right) .
$$

Since $b-\beta_{h}(b) \in \Gamma_{h}\left(\mathfrak{M}^{\prime} \times \mathfrak{u}\right)$, it would be enough to prove that $f(h, a)=0$ if $a \in \Gamma_{h}\left(\mathfrak{M}^{\prime} \times \mathfrak{U}\right)$. It is clearly sufficient to consider the case when

$$
a=\Gamma_{h}\left(X_{1} \cdots X_{r} \times H_{1} H_{2} \cdots H_{s}\right)
$$

where $X_{1}, \cdots, X_{r} \in \mathfrak{m} \cap \mathfrak{g}_{0}(r \geqq 1)$ and $H_{1}, \cdots, H_{s} \in \mathfrak{h}_{0}(s \geqq 0)$. Put $m(u)$ $=\exp \left(u_{1} X_{1}\right) \cdots \exp \left(u_{r} X_{r}\right), h(t)=\exp \left(t_{1} H_{1}+\cdots+t_{s} H_{s}\right)\left(u_{i}, t_{j} \in R\right)$. Then as we have seen in $\$ 5$, 


$$
f(h, a)=\left\{\left(\partial^{r+s} / \partial u_{1} \cdots \partial u_{r} \partial t_{1} \cdots \partial t_{s}\right) f\left(m(u) h h(t)(m(u))^{-1}\right)\right\}_{u_{i}=t_{j}=0} .
$$

But since $f$ is an invariant function and $r \geqq 1$, the right-hand side is zero. This proves the lemma.

CoROLlary. Let $g$ be the restriction on $A^{\prime}$ of a function $f \in I^{\infty}(' M)$. Then if $b \in \mathfrak{B}, \delta(b) f$ coincides with $\beta(b) g$ on $A^{\prime}$.

Let $h$ be a point in $A^{\prime}$. Then $g(h, \beta(b))=g\left(h, \beta_{h}(b)\right)=f\left(h, \beta_{h}(b)\right)$ and $f(h, \delta(b))=f\left(h, \delta_{h}(b)\right)$. Therefore, in view of the above lemma, it would be enough to show that $\beta_{h}\left(\delta_{h}(b)\right)=\beta_{h}(b)$. But

$$
b-\delta_{h}(b) \in \Gamma_{h}\left(\mathfrak{Q}^{\prime} \times \mathfrak{M}\right)
$$

and $\mathfrak{M}=\Gamma_{h}\left(\mathfrak{S}_{-} \times \mathfrak{u}\right)$ from Lemma 22 . Therefore

$$
\begin{aligned}
\Gamma_{h}\left(\mathfrak{Q}^{\prime} \times \mathfrak{M}\right) & =\Gamma_{h}\left(\mathfrak{Q}^{\prime} \times \Gamma_{h}\left(\mathfrak{S}_{-} \times \mathfrak{u}\right)\right) \\
& =\Gamma_{h}\left(\mathfrak{Q}^{\prime} \mathfrak{S}_{-} \times \mathfrak{U}\right) \subset \Gamma_{h}\left(\mathfrak{B}^{\prime} \times \mathfrak{u}\right)
\end{aligned}
$$

from the corollary to Lemma 15 . On the other hand

$$
\delta_{h}(b)-\beta_{h}\left(\delta_{h}(b)\right) \in \Gamma_{h}\left(\mathfrak{B}^{\prime} \times \mathfrak{U}\right)
$$

and therefore

$$
b-\beta_{h}\left(\delta_{h}(b)\right) \in \Gamma_{h}\left(\mathfrak{B}^{\prime} \times \mathfrak{U}\right) .
$$

This proves that $\beta_{h}(b)=\beta_{h}\left(\delta_{h}(b)\right)$.

8. Determination of $\beta(z)$ in terms of the invariants. As before ( $\S 6)$ let $W$ and $W_{-}$respectively be the Weyl groups of $\mathfrak{g}$ and $\mathfrak{m}$ (relative to $\mathfrak{h}$ ) and let $J$ and $J_{-}$be the corresponding subalgebras of invariants of $\mathfrak{u}$. In $\S 6$ we have defined the isomorphisms $\gamma$ and $\gamma_{-}$of 8 and $Z_{M}$ onto $J$ and $J_{-}$respectively. (We recall that $\mathfrak{Z}_{M}$ is the center of $\mathfrak{M}$.) The operators $\beta(z)$ and $\beta(w)$ can be expressed in terms of $\gamma(z)$ and $\gamma_{-}(w)\left(z \in Z, w \in Z_{M}\right)$ as follows.

Theorem 2. If $z \in \mathbb{Z}$ and $w \in Z_{M}$ then

$$
\beta(z)=|d|^{-1 / 2} \gamma(z) \circ|d|^{1 / 2}, \quad \beta(w)=\left|d_{-}\right|^{-1 / 2} \gamma_{-}(w) \circ\left|d_{-}\right|^{1 / 2}
$$

as differential operators on $A^{\prime}$. (Here o denotes operator product.)

Assuming this for a moment, we shall now prove Lemma 21 and thus complete the proof of Theorem 1. Let $z \in B$ and $f \in I_{c}^{\infty}(' M)$. Then as we have seen in $\S 6$, it is enough to prove that $\Theta f$ is zero on $A^{\prime}$. Let $g$ denote the restriction of $f$ on $A^{\prime}$. Then by the corollary to Lemma 25 and Theorem $2, \delta(z) f$ coincides on $A^{\prime}$ with $\beta(z) g=|d|^{-1 / 2} \gamma(z)\left(|d|^{1 / 2} g\right)$. On the other hand $\gamma_{-}(\mu(z))$ $=\gamma(z)$. Therefore again from Theorem 2 and the corollary to Lemma 25, $\left|d_{+}\right|^{-1 / 2} \mu(z)\left(\left|d_{+}\right|{ }^{1 / 2} f\right)$ coincides on $A^{\prime}$ with $|d|^{-1 / 2} \gamma(z)\left(|d|^{1 / 2} g\right)$ since $d=d_{+} d_{-}$. This proves that $\Theta f$ vanishes identically on $A^{\prime}$. 
We now come to the proof of Theorem 2. Put

$$
\begin{aligned}
& \Phi=\beta(z)-|d|^{-1 / 2} \gamma(z) \circ|d|^{1 / 2}, \\
& \Psi=\beta(w)-\left|d_{-}\right|^{-1 / 2} \gamma_{-}(w) \circ\left|d_{-}\right|^{1 / 2} .
\end{aligned}
$$

Then we have to show that $\Phi=\Psi=0$. Put $G^{*}=\operatorname{Ad}(G) \simeq G / Z$ and $x^{*}=\operatorname{Ad}(x)$ $(x \in G)$. It is obvious from its definition (see Lemma 15) that $\Gamma_{m}$ depends only on $m^{*}(m \in M)$ and therefore $\beta_{h}(b)(b \in \mathfrak{B})$ only on $h^{*}\left(h \in A^{\prime}\right)$. Since the same holds for $d(h)$ and $d_{-}(h)$, we can regard $\Phi$ and $\Psi$ as differential operators on $A^{\prime *}$ and it would clearly be enough to prove that $\Phi=\Psi=0$ on $A^{\prime *}$. Let $G_{0}$ be any connected Lie group whose Lie algebra is $g_{0}$. Then the adjoint groups of $G$ and $G_{0}$ are the same and hence the above argument shows that it is permissible, for the proof of our theorem, to replace $G$ by $G_{0}$. Therefore if $G_{c}$ is the simply connected complex analytic group with the Lie algebra $\mathfrak{g}$, we may assume that $G$ is the real analytic subgroup of $G_{c}$ corresponding to $g_{0}$. Let $A_{c}$ and $M_{c}$ be the complex analytic subgroups which correspond to $\mathfrak{h}$ and $\mathrm{m}$ respectively. Then we know from Lemmas 3 and 4 that $A \subset A_{c}$ and therefore (see Lemma 7) $M \subset M_{c}$. Now $\mathfrak{h}$, regarded as an additive group, is locally isomorphic to $A_{c}$ under the exponential mapping and so it is a complex Lie group whose Lie algebra is also $\mathfrak{h}$. Therefore elements of $\mathfrak{U}$ may now be considered as (holomorphic) differential operators on $\mathfrak{h}$. Let $\mathfrak{h}_{1}$ be the complete inverse image of $A$ (under the exponential mapping) in $\mathfrak{h}$. Then $\mathfrak{h}_{1}$ is closed and therefore it is a (real) Lie subgroup and its Lie algebra is $\mathfrak{h}_{0}$. Put

$$
\begin{aligned}
\Delta(H) & =\prod_{\alpha \in P}\left(e^{\alpha(H) / 2}-e^{-\alpha(H) / 2}\right), \\
\Delta_{-}(H) & =\prod_{\alpha \in P_{-}}\left(e^{\alpha(H) / 2}-e^{-\alpha(H) / 2}\right)
\end{aligned}
$$

Then it is obvious that

$$
\begin{aligned}
d(\exp H) & =(-1)^{r} \Delta(H)^{2}, \\
d_{-}(\exp H) & =(-1)^{s} \Delta_{-}(H)^{2}
\end{aligned}
$$

where $r$ and $s$ are the numbers of roots in $P$ and $P_{-}$respectively. For any function $\lambda$ on $\mathfrak{h}$ and $s \in W$, let $\lambda^{s}$ denote the function $H \rightarrow \lambda\left(s^{-1} H\right)(H \in \mathfrak{h})$. (If $\lambda$ is a linear function we write $s \lambda$ instead of $\lambda^{s}$.) Then for any root $\alpha, s \alpha$ is also a root and therefore $\Delta^{s}=\epsilon(s) \Delta$ where $\epsilon(s)= \pm 1$. Similarly if $s \in W_{-}$, it is clear that $\Delta_{-}^{s}=\epsilon_{-}(s) \Delta_{-}$where again $\epsilon_{-}(s)= \pm 1$. But if $s_{\alpha}$ is the Weyl reflexion corresponding to a root $\alpha \in P_{-}$, it is known (see Weyl [14(b)]) that $\epsilon\left(s_{\alpha}\right)=\epsilon_{-}\left(s_{\alpha}\right)=-1$. Therefore since $\epsilon$ and $\epsilon_{-}$are both homomorphisms, it follows that $\boldsymbol{\epsilon}(s)=\epsilon_{-}(s)$ for all $s \in W_{-}$. This shows in particular that if

$$
\Delta_{+}(H)=\prod_{\alpha \in P_{0} \cup P_{+}}\left(e^{\alpha(H) / 2}-e^{-\alpha(H) / 2}\right) \quad(H \in \mathfrak{h}),
$$

then $\Delta_{+}^{s}=\Delta_{+}$for all $s \in W_{-}$. (We shall need this result a little later in $§ 10$.) 
Consider the open submanifold $\mathfrak{h}^{\prime}$ of $\mathfrak{h}$ consisting of those points $H$ where $\Delta(H) \neq 0$. Then $\mathfrak{h}_{\mathfrak{1}}^{\prime}=\mathfrak{h}^{\prime} \cap \mathfrak{h}_{1}$ is the complete inverse image of $A^{\prime}$. We regard $\mathfrak{h}_{1}^{\prime}$ as an open submanifold of the real Lie group $\mathfrak{h}_{1}$. It is obvious that corresponding to any differential operator $D$ on $A^{\prime}$ there exists exactly one differential operator $\bar{D}$ on $\mathfrak{h}_{1}^{\prime}$ with the following property. If $f \in C^{\infty}\left(A^{\prime}\right)$ and $\bar{f}(H)=f(\exp H) .\left(H \in \mathfrak{h}_{1}^{\prime}\right)$, then $\bar{f}(H, \bar{D})=f(\exp H, D)$. Moreover $\bar{D}=0$ if and only if $D=0$. Hence it would be sufficient to prove that $\bar{\Phi}=\bar{\Psi}=0$. It is clear that

$$
\begin{aligned}
& \bar{\Phi}=\overline{\beta(z)}-\Delta^{-1} \gamma(z) \circ \Delta, \\
& \bar{\Psi}=\overline{\beta(w)}-\Delta_{-}^{-1} \gamma_{-}(w) \circ \Delta_{-} .
\end{aligned}
$$

Let $F$ and $F_{-}$be the additive groups generated by the roots $\alpha$ in $P$ and $P_{-}$ respectively. Then it follows from the corollaries to Lemmas 23 and 24 that we can choose elements $\sigma_{1}, \cdots, \sigma_{p} \in F, \tau_{1}, \cdots, \tau_{q} \in F_{-}, u_{1}, \cdots, u_{p}$, $v_{1}, \cdots, v_{q} \in \mathfrak{U}$ and two integers $r, k \geqq 0$ such that

$$
\begin{aligned}
& \bar{\Phi}=\Delta^{-r} \sum_{i=1}^{p} e^{\sigma_{i}} u_{i} \\
& \bar{\Psi}=\Delta_{-}^{-k} \sum_{j=1}^{q} e^{\tau_{i} v_{j} .}
\end{aligned}
$$

Now suppose $\Phi \neq 0$. We may assume that $\sigma_{1}, \cdots, \sigma_{p}$ are all distinct and $u_{i} \neq 01 \leqq i \leqq p$. Let $\mathfrak{F}_{R}$ be the space consisting of all real linear combinations of the roots $\alpha$. Then a lexicographic order is defined in $\mathfrak{F}_{R}$ (see $\$ 2$ ) and hence $F$ and $F_{-}$are ordered groups. Let $\sigma_{1}$ be the highest element among $\left(\sigma_{1}, \cdots, \sigma_{p}\right)$. Put $\rho=2^{-1} \sum_{\alpha \in P} \alpha$ and suppose $\nu$ is an irreducible representation of $g$ on a finite-dimensional space $V$ with the highest weight $\Lambda$. We denote the corresponding representations of $\mathfrak{B}$ and $G_{c}$ also by $\nu$. Let $\chi$ be the character of $\nu$ on $G_{c}$. Then if $\Lambda^{\prime}=\Lambda+\rho$ one knows (see Weyl [14(a)]) that

$$
\chi(\exp H) \Delta(H)=\sum_{s \in W} \epsilon(s) e^{s \Lambda^{\prime}(H)}
$$

On the other hand $\chi\left(x y x^{-1}\right)=\chi(y)\left(x, y \in G_{c}\right)$ and so as we have seen during the proof of Lemma $25, \chi(h, a)=0$ if $h \in A$ and $a \in \Gamma_{h}\left(\mathfrak{B}^{\prime} \times \mathfrak{u}\right)$. This implies that $\chi(h, b)=\chi\left(h, \beta_{h}(b)\right)\left(b \in \mathfrak{B}, h \in A^{\prime}\right)$ and therefore if $\bar{\chi}(H)=\chi(\exp H)$ $(H \in \mathfrak{h})$ and $\beta_{H}(b)$ denotes the local expression of $\overline{\beta(b)}$ at $H \in \mathfrak{h}_{1}^{\prime}$, it follows that

$$
\bar{\chi}\left(H, \beta_{H}(z)\right)=\chi(\exp H, z) .
$$

Now $\sigma$ being a linear function on $\mathfrak{h}$, we may extend it (uniquely) to a homomorphism of $\mathfrak{U}$ into $C$ such that $\sigma(1)=1$. Then it is known (see [6(b), p. 73]) that $\nu(z) \phi=\Lambda^{\prime}(\gamma(z)) \phi$ for any $\phi \in V$ and therefore 


$$
z \chi=\Lambda^{\prime}(\gamma(z)) \chi .
$$

In view of our result above, this implies that

$$
\bar{\chi}\left(H, \beta_{H}(z)\right)=\Lambda^{\prime}(\gamma(z)) \bar{\chi}(H)
$$

Put $\chi^{\prime}=\bar{\chi} \Delta$. Then since $\gamma(z) \in J$, it follows from the formula for $\chi^{\prime}$ given above that

$$
\chi^{\prime}(H, \gamma(z))=\Lambda^{\prime}(\gamma(z)) \chi^{\prime}(H) \quad(H \in \mathfrak{h})
$$

Therefore

$$
\bar{\chi}(H, \bar{\Phi})=\bar{\chi}\left(H, \beta_{H}(z)\right)-\Delta(H)^{-1} \chi^{\prime}(H, \gamma(z))=0
$$

for $H \in \mathfrak{h}_{1}^{\prime}$. On the other hand let $\Lambda=\Lambda_{1}, \cdots, \Lambda_{N}$ be all the distinct weights of $\nu$ and let $n_{i}$ be the multiplicity of $\Lambda_{i}(1 \leqq i \leqq N)$. Then $\Lambda_{i} \in \mho_{R}$ and $\Lambda_{i}<\Lambda$ $2 \leqq i \leqq N$ and

$$
\bar{\chi}(H)=\chi(\exp H)=\sum_{i=1}^{N} n_{i} e^{\Lambda_{i}(H)}
$$

Therefore it follows from our formula for $\bar{\Phi}$ that

$$
0=\bar{\chi}(H, \bar{\Phi})=\Delta(H)^{-r} \sum_{i=1}^{p} \sum_{j=1}^{N} n_{j} \Lambda_{j}\left(u_{i}\right) \exp \left(\sigma_{i}(H)+\Lambda_{j}(H)\right)
$$

for all $H \in \mathfrak{h}_{1}^{\prime}$. But since $\mathfrak{h}_{\mathfrak{1}}^{\prime}$ contains the nonempty open subset $\mathfrak{h}_{0} \cap \mathfrak{h}^{\prime}$ of $\mathfrak{h}_{0}$, it is obvious that a meromorphic function on $\mathfrak{h}$ cannot vanish everywhere on $\mathfrak{h}_{\mathfrak{1}}^{\prime}$ unless it is identically zero. Therefore

$$
\sum_{i=1}^{p} \sum_{j=1}^{N} n_{j} \Lambda_{j}\left(u_{i}\right) e^{\sigma_{i}+\Lambda_{i}}=0 .
$$

But $\sigma_{1}+\Lambda=\sigma_{1}+\Lambda_{1}>\sigma_{i}+\Lambda_{j}$ unless $i=j=1$ and the exponentials of distinct linear functions are linearly independent (see for example Lemma 41 of [6(b) ]). So it follows that $\Lambda\left(u_{1}\right)=0$. On the other hand if $l=\operatorname{dim}_{c} \mathfrak{h}$, there exist $l$ linearly independent elements $\lambda_{1}, \cdots, \lambda_{l} \in \mathfrak{F}_{R}$ such that for any set $\left(m_{1}, \cdots, m_{l}\right)$ of non-negative integers $\Lambda_{0}=m_{l} \lambda_{l}+\cdots+m_{l} \lambda_{l}$ is the highest weight of some irreducible representation of $\mathfrak{g}$ (see [6(b), Theorem 1]). Therefore $\Lambda_{0}\left(u_{1}\right)=0$ for all such $\Lambda_{0}$ and from this we can conclude (see Lemma 22 of $[6(\mathrm{~b})])$ that $u_{1}=0$. However this contradicts our hypothesis and so $\bar{\Phi}=0$.

Now we come to $\bar{\Psi}$. Again assuming that $\bar{\Psi} \neq 0$, we may suppose that $\tau_{1}>\tau_{2}>\cdots>\tau_{q}$ in $F_{-}$and $v_{j} \neq 0 \quad 1 \leqq j \leqq q . \nu, \Lambda$ and $V$ being as above, let $\phi \neq 0$ be a vector in $V$ belonging to the weight $\Lambda$. Then if $U=\nu(\mathfrak{M}) \phi$, we get a representation $\nu_{1}$ of $M_{c}$ on $U$ which is irreducible (see Lemma 2 of [6(f)]). Obviously $\Lambda$ is the highest weight of $\nu_{1}$. Hence if $\chi_{1}$ is the character of $\nu_{1}$ and $\Lambda^{\prime \prime}=\Lambda+\rho_{-}\left(\right.$where $\left.\rho_{-}=2^{-1} \sum_{\alpha \in P_{-}} \alpha\right)$, we have the formula (see Weyl [14(a)]) 


$$
\chi_{1}(\exp H) \Delta_{-}(H)=\sum_{s \in W} \epsilon(s) e^{\Delta^{\prime \prime}(s H)}
$$

We can now apply the above argument to $\chi_{1}$ (instead of $\chi$ ) and conclude that $\Lambda\left(v_{1}\right)=0$. Again this implies that $v_{1}=0$ and so we get a contradiction. Hence Theorem 2 is now proved.

9. Eigen-distributions on ' $M$. Let $\tau$ be an invariant distribution on ' $M$. Since $\delta(z)(z \in 3)$ is an invariant differential operator on ' $M$ (see the corollary to Lemma 17), $\delta(z) \tau$ is also invariant. Therefore it follows from Theorem 1 that $\delta\left(z_{1} z_{2}\right) \tau=\delta\left(z_{1}\right) \delta\left(z_{2}\right) \tau\left(z_{1}, z_{2} \in Z\right)$. We shall say that $\tau$ is an eigen-distribution of $\mathbb{Z}$ if it is an eigen-distribution of $\delta(z)$ for every $z \in \mathfrak{Z}$. In case this is so, it is clear that there exists a homomorphism $\chi$ of $B$ into $C$ such that $\delta(z) \tau$ $=\chi(z) \tau(z \in 3)$. The following theorem is the main result of the above theory.

TheOREM 3. Let $\tau$ be an invariant distribution on ' $M$. Then if it is an eigendistribution of 3, it coincides with an analytic function on ' $M$.

Define $J$ and $J_{-}$as in $\S 8$. Then $J_{-} \supset J$ and since $W$ is a finite group, it follows from the theory of invariants (see Weyl [14(c), p. 275]) that $J$ has a finite number of generators (over $C$ ) and $\mathfrak{U}$ is a finite module over $J$ (see [6(c), p. 200]). Therefore $J$ is a Noetherian ring and hence by a well-known theorem in algebra (see van der Waerden $\left[13\right.$, vol. II, §99]) $J_{-}$is also a finite module over $J$. Hence any element $u^{\prime} \in J_{-}$satisfies an equation of the form

$$
w^{\prime g}+\gamma\left(z_{1}\right) w^{\prime \theta-1}+\cdots+\gamma\left(z_{\theta}\right)=0
$$

where $z_{1}, \cdots, z_{g} \in \mathcal{B}$ and $g$ is a positive integer. Now the notation being as in $\S 8$, let $w \in 3_{M}$ and put $w^{\prime}=\gamma_{-}(w)$. Then by applying the isomorphism $\gamma_{-}^{-1}$ to the above equation, we get

$$
w^{0}+\mu\left(z_{1}\right) w^{o-1}+\cdots+\mu\left(z_{o}\right)=0 .
$$

Now put $\tau^{\prime}=\left|d_{+}\right|^{1 / 2} \tau$. If $\chi$ is the homomorphism of $\mathbb{Z}$ into $C$ corresponding to $\tau$, it follows from Theorem 1 that $\mu(z) \tau^{\prime}=\chi(z) \tau^{\prime}(z \in Z)$ and therefore since $3_{M}$ is abelian,

$$
w^{\sigma} \tau^{\prime}+\chi\left(z_{1}\right) w^{g-1} \tau^{\prime}+\cdots+\chi\left(z_{g}\right) \tau^{\prime}=0 .
$$

Let $H_{1}, \cdots, H_{p}$ be a base for $\mathfrak{h}_{0} \cap \mathfrak{p}$ over $R$. Since $-B(X, X)\left(X \in \mathfrak{f}_{0}\right)$ is a positive-definite quadratic form on $\mathfrak{t}_{0}$, we can choose a base $X_{1}, \cdots, X_{r}$ for $\mathfrak{m}_{t_{0}}$ over $R$ such that $-B\left(X_{i}, X_{j}\right)=\delta_{i j}(1 \leqq i, j \leqq r)$ where $\delta_{i j}=1$ or 0 according as $i=j$ or not. Put

$$
\square=H_{1}^{2}+\cdots+H_{p}^{2}+X_{1}^{2}+X_{2}^{2}+\cdots+X_{r}^{2} .
$$

It is well known that $X_{1}^{2}+\cdots+X_{r}^{2}$ lies in $Z_{M}$ and therefore the same holds for $\square$. Hence in view of what we have said above, $\tau^{\prime}$ satisfies an equation of the form

$$
\square^{o} \tau^{\prime}+c_{1} \square^{o-1} \tau^{\prime}+\cdots+c_{o} \tau^{\prime}=0
$$


where $c_{1}, \cdots, c_{0}$ are complex numbers. But it is obvious from its definition that the analytic differential operator $\square$ is of the elliptic type (see Gårding [3]) everywhere on $M$. The same therefore holds for

$$
\square^{0}+c_{1} \square^{0-1}+\cdots+c_{0}
$$

and so we can conclude from the work of Schwartz $[11$, p. 136] and John [8] (see also [3]) on the generalization of the classical Bernstein Theorem to distributions, that $\tau^{\prime}$, and hence also $\tau$ are analytic functions on ' $M$. This proves the theorem.

We shall now try to determine $\tau$. Put ' $A={ }^{\prime} M \cap A$. Then it is obvious from Lemma 7 that ' $M=\bigcup_{m \in M} m^{\prime} A m^{-1}$ and since $\tau$ is an invariant analytic function, it would be enough to know its restriction on $A^{\prime}=A \cap G^{\prime}$ (see Lemma 8). Let $\sigma^{\prime}$ denote the restriction of $\tau^{\prime}=\left|d_{+}\right|{ }^{1 / 2} \tau$ on $A^{\prime}$. Since $\mu(z) \tau^{\prime}=\chi(z) \tau^{\prime}(z \in B)$, we conclude from the corollary to Lemma 25 that $\beta(\mu(z)) \sigma^{\prime}=\chi(z) \sigma^{\prime}$. On the other hand

$$
\beta(\mu(z))=\left|d_{-}\right|^{-1 / 2} \gamma(z) \circ\left|d_{-}\right|^{1 / 2}
$$

from Theorem 2. Therefore if $\sigma(h)=|d(h)|^{1 / 2} \tau(h)\left(h \in A^{\prime}\right)$,

$$
\gamma(z) \sigma=\chi(z) \sigma \quad(z \in B) \text {. }
$$

Let us now assume that $\tau \neq 0$ so that $\sigma \neq 0$ and $\chi(1)=1$. Then from Theorem 5 of $[6(b)]$, there exists a linear function $\Lambda$ on $\mathfrak{h}$ such that $\chi(z)=\Lambda(\gamma(z))$ for every $z \in 3$. Moreover the linear functions $s \Lambda(s \in W)$ are the only ones which have this property so that $\Lambda$ is uniquely determined by $\chi$ up to an operation of $W$. Since $\gamma$ is an isomorphism of $\mathbb{Z}$ onto $J$ we obtain the following differential equations for $\sigma$.

$$
v \sigma=\Lambda(v) \sigma \quad(v \in J) .
$$

Define $u^{\bullet}(u \in \mathfrak{U}, s \in W)$ as in $\S 6$. Then $\mathfrak{U}$ being abelian, we can, for any $u \in \mathfrak{U}$, consider the polynomial

$$
\prod_{s \in W}\left(\zeta-u^{s}\right)=\zeta^{w}+v_{1} \zeta^{w-1}+\cdots+v_{w}
$$

in the indeterminate $\zeta$ with coefficients in $J$. (Here $w$ is the order of $W$.) Replacing $\zeta$ by $u$, we obtain the identity

$$
u^{w}+u^{w-1} v_{1}+\cdots+v_{w}=0
$$

which when applied to $\sigma$ gives

$$
u^{w} \sigma+\Lambda\left(v_{1}\right) u^{w-1} \sigma+\cdots+\Lambda\left(v_{w}\right) \sigma=0 .
$$

On the other hand since $\Lambda$ is a homomorphism of $\mathfrak{u}$, it is obvious that

$$
\prod_{. \in W}\left(\zeta-\Lambda\left(u^{s}\right)\right)=\zeta^{w}+\Lambda\left(v_{1}\right) \zeta^{w-1}+\cdots+\Lambda\left(v_{w}\right) .
$$


Therefore the above equation can be written in the form

$$
\prod_{s \in W}\left(u-\Lambda\left(u^{s}\right)\right) \sigma=0 .
$$

Thus we have obtained the following result.

LEMMA 26. Let $\sigma(h)=|d(h)|^{1 / 2} \tau(h)\left(h \in A^{\prime}\right)$ where $\tau$ is the analytic function (on ' $M$ ) of Theorem 3. Then there exists a linear function $\Lambda$ on $\mathfrak{h}$ such that

$$
v \sigma=\Lambda(v) \sigma
$$

for every $v \in J$. Moreover if $\tau \neq 0, \Lambda$ is unique up to an operation of $W$ and

$$
\prod_{\bullet \in W}\left(u-\Lambda\left(u^{s}\right)\right) \sigma=0
$$

for all $u \in \mathfrak{U}$.

In order to obtain more precise information about $\sigma$ from the above lemma we first need some simple facts about differential equations of a certain type. Let $I$ be an open interval on the real line $R$. We denote the variable on $R$ by $t$ and the differential operator $d / d t$ by $\partial$. Let $P(\zeta) \neq 0$ be a polynomial in an indeterminate $\zeta$ with complex coefficients.

Lemma 27. Let $\xi$ be an analytic function on $I$ such that $P(\partial) \xi=0$. Then $\xi(t)=p_{1}(t) e^{\lambda_{1} t}+\cdots+p_{s}(t) e^{\lambda_{s} t}(t \in I)$ where $p_{1}, \cdots, p_{s}$ are polynomials (with complex coefficients) and $\lambda_{1}, \cdots, \lambda_{s}$ are all the distinct roots of the equation $P(\zeta)=0$. If $\mu_{i}$ is the multiplicity of the root $\lambda_{i}$ of $P$, the degree of $p_{i}$ is less than $\mu_{i}(1 \leqq i \leqq s)$.

Although this lemma is undoubtedly known, we give a short proof for the sake of completeness. Without loss of generality we may assume that the origin lies in $I$. Let $r$ be the degree of $P$. First we claim that if $\left(\partial^{i \xi}\right)_{t=0}=0$, $0 \leqq j \leqq r$, then $\xi=0$. For otherwise if $t^{m}$ is the lowest power of $t$ appearing in the Taylor expansion $\xi(t)=a_{0} t^{m}+a_{1} t^{m+1}+\cdots$ around $t=0\left(a_{i} \in C, a_{0} \neq 0\right)$, it is clear that $m>r$ and therefore the coefficient of $t^{m-r}$ in the Taylor expansion of $P(\partial) \xi$ is not zero. Hence it follows easily that any $r+1$ solutions of the differential equation $P(\partial) \Xi=0$ which are analytic on $I$ must be linearly dependent over $C$. On the other hand it is easy to verify that the functions $t^{q} e^{\lambda_{i} t}\left(0 \leqq q<\mu_{i}, 1 \leqq i \leqq s\right)$ constitute a set of $r$ linearly independent solutions of this equation. Hence $\xi$ must be a linear combination of them.

Corollary. Let $U$ be an open connected set in a Euclidean space $E$ of dimension $l$ over $R$ and let $\left(t_{1}, \cdots, t_{l}\right)$ denote the Cartesian coordinates in $E$. Put $\partial_{i}=\partial / \partial t_{i}(i=1, \cdots, l)$ and let $P_{1}(\zeta), \cdots, P_{l}(\zeta)$ be nonzero polynomials of degrees $r_{1}, \cdots, r_{l}$ respectively in the indeterminate $\zeta$ with coefficients in $C$. Consider an analytic function $\xi$ on $U$ such that $P_{i}\left(\partial_{i}\right) \xi=0(1 \leqq i \leqq l)$. Then $\xi$ is a linear combination with complex coefficients of functions of the form 


$$
t_{1}^{m_{1}} t_{2}^{m_{2}} \cdots t_{l}^{m_{l}} e^{\lambda_{1} t_{1}+\cdots+\lambda_{l} t_{l}}
$$

where $m_{1}, \cdots, m_{l}$ are integers, $\lambda_{i}$ is a root of the equation $P_{i}(\zeta)=0$ of multiplicity $\mu_{i}$ and $0 \leqq m_{i}<\mu_{i}(1 \leqq i \leqq l)$.

Put $r=r_{1} r_{2} \cdots r_{l}$. It is easy to verify that the functions written above form a system of $r$ linearly independent solutions of our differential equations (see Lemma 41 of [6(b) ]). Hence it is enough to prove that any $r+1$ analytic solutions are linearly dependent. Again we may assume that the origin lies in $U$. Then the equations $\left(\partial_{1}^{j_{1}} \partial_{2}^{j_{2}} \cdots \partial_{l}^{j_{l} \xi}\right)_{t_{1}=\cdots=t_{l}=0}=0,0 \leqq j_{i}<r_{i}, 1 \leqq i \leqq l$ are exactly $r$ in number. Hence given $r+1$ analytic solutions $\xi_{1}, \cdots, \xi_{r+1}$, we can choose $c_{i} \in C(1 \leqq i \leqq r+1)$, not all zero, such that $\xi=c_{1} \xi_{1}+\cdots+c_{r+1} \xi_{r+1}$ satisfies these $r$ conditions. We shall now prove that $\xi=0$. Since $\xi$ is analytic it is enough to show that it is zero in some neighborhood of the origin. Hence it would be sufficient to prove the following lemma.

Lemma 28. Let $\epsilon$ be a positive real number and $U$ the cube $\left|t_{i}\right|<\epsilon(1 \leqq i \leqq l)$ in E. Suppose $\xi$ is an analytic function on $U$ such that $P_{i}\left(\partial_{i}\right) \xi=01 \leqq i \leqq l$ and

$$
\left(\partial_{1}^{j_{1}} \partial_{2}^{j_{2}} \cdots \partial_{l}^{j_{l}} \xi\right)_{t_{1}=\cdots=t_{l}=0}=0
$$

$0 \leqq j_{1}<r_{1}, \cdots, 0 \leqq j_{l}<r_{l}$. Then $\xi=0$.

We use induction on $l$. If $l=1$ we have seen during the proof of Lemma 27 that our assertion is true. So assume $l \geqq 2$ and let $\bar{U}$ denote the section of $U$ defined by $t_{l}=0$. Let $\bar{\xi}_{j}$ be the restriction of $\xi_{j}=\partial_{l}^{j} \xi$ on $\bar{U}\left(0 \leqq j<r_{l}\right)$. Then it follows from our induction hypothesis that $\bar{\xi}_{j}$ is identically zero. For fixed $t_{1}, \cdots, t_{l-1}\left(\left|t_{i}\right|<\epsilon, i=1, \cdots, l-1\right)$ put $\eta(t)=\xi\left(t_{1}, \cdots, t_{l-1}, t\right)(|t|<\epsilon)$. Then $\left(\partial^{j} \eta\right)_{t=0}=\bar{\xi}_{j}\left(t_{1}, \cdots, t_{l-1}\right)=0$ and $P_{l}(\partial) \eta=0$ where $\partial=d / d t$. Therefore $\eta=0$ by the case $l=1$ and so this proves that $\xi=0$ on $U$.

After this preparation we return to our problem of determining $\sigma$. Let $h_{0}$ be a point in $A^{\prime}$. We can choose an open connected neighborhood $U$ of zero in the real Euclidean space $\mathfrak{h}_{0}$ such that $h_{0} \exp H \in A^{\prime}$ for all $H \in U$. Then $F(H)=\sigma\left(h_{0} \exp H\right)(H \in U)$ is an analytic function on $U$. As in $\S 8$ we can regard elements of $\mathfrak{U}$ as differential operators on $\mathfrak{h}_{0}$ and therefore also on $U$. Then it follows from Lemma 26 that

$$
\prod_{s \in W}\left(u-\Lambda\left(u^{s}\right)\right) F=0
$$

and

$$
(v-\Lambda(v)) F=0
$$

Let $H_{1}, \cdots, H_{l}$ be a base for $\mathfrak{h}_{0}$ over $R$ and let $\left(t_{1}, \cdots, t_{l}\right)$ denote the corresponding Cartesian coordinates in $\mathfrak{h}_{0}$. Then $\partial_{i}=H_{i}$ and if

$$
P_{i}(\zeta)=\prod_{s \in W}\left(\zeta-s \Lambda\left(H_{i}\right)\right)
$$


it is obvious that $P_{i}\left(\partial_{i}\right) F=P_{i}\left(H_{i}\right) F=0(1 \leqq i \leqq l)$. Moreover $s \Lambda\left(H_{i}\right)(s \in W)$ are exactly the roots of the polynomial $P_{i}(\zeta)$. Hence it follows from the corollary to Lemma 27 that

$$
F=p_{1} e^{\lambda_{1}}+\cdots+p_{r} e^{\lambda_{r}}
$$

where $p_{1}, \cdots, p_{r}$ are polynomial functions and $\lambda_{1}, \cdots, \lambda_{r}$ linear functions on $\mathfrak{h}$. We may assume that $\lambda_{1}, \cdots, \lambda_{r}$ are all distinct and $p_{i} \neq 0(1 \leqq i \leqq r)$. It is obvious that $u e^{\lambda}=\lambda(u) e^{\lambda}(u \in \mathfrak{U})$ for any linear function $\lambda$ on $\mathfrak{h}$. Hence if $v \in J$,

$$
0=(v-\Lambda(v)) F=\sum_{i=1}^{r} p_{i}^{\prime} e^{\lambda_{i}}
$$

where $p_{i}^{\prime}=\left(\lambda_{i}(v)-\Lambda(v)\right) p_{i}+q_{i}$ and $q_{i}$ is a polynomial of lower degree than $p_{i}$. But the exponentials of distinct linear functions are known to be linearly independent over the ring of polynomial functions (see Lemma 41 of [6(b) ]). Therefore $p_{i}^{\prime}=0$ and so

$$
\lambda_{i}(v)=\Lambda(v)
$$$$
(1 \leqq i \leqq r) .
$$

This being true for every $v \in J$, it follows from Theorem 5 of [6(b)] that $\lambda_{i}=s_{i} \Lambda$ for some $s_{i} \in W$. Now let $W_{\Lambda}$ be the subgroup consisting of those elements $s \in W$ for which $s \Lambda=\Lambda$. Let $N$ be the order of $W_{\Lambda}$.We shall prove that the degree of each $p_{i}$ is smaller than $N$. For otherwise suppose the degree of $p_{1}$ is $n_{1}$ and $n_{1} \geqq N$. It is easily seen that we can choose an element $H_{0} \in \mathfrak{h}_{0}$ such that $\Lambda\left(H_{0}\right) \neq s \Lambda\left(H_{0}\right)$ ( $\left.s \in W\right)$ unless $s \in W_{\Lambda}$ and the degree of the polynomial $p(t)=p_{1}\left(t H_{0}\right)$ in $t$ is $n_{1}$. Put $f(t)=F\left(t H_{0}\right)$. Then $f$ is an analytic function defined on some open interval $I$ in $R$ containing the origin. On the other hand, considering $H_{0}$ as a differential operator on $U$, we have the relation

$$
F\left(t H_{0}, \prod_{s \in W}\left(H_{0}-s \Lambda\left(H_{0}\right)\right)\right)=0
$$

From this it follows immediately that

$$
\prod_{\delta \in W}\left(\partial-s \Lambda\left(H_{0}\right)\right) f=0
$$

where $\partial=d / d t$. Put $p_{i}^{\prime}(t)=p_{i}\left(t H_{0}\right)$ and $\lambda_{i}^{\prime}=\lambda_{i}\left(H_{0}\right)$. Then

$$
f(t)=\sum_{i=1}^{r} p_{i}^{\prime}(t) e^{\lambda_{i}^{\prime} t} .
$$

Since $\lambda_{1}^{\prime}, \cdots, \lambda_{r}^{\prime}$ are all distinct $e^{\lambda^{\lambda_{1}} t}, \cdots, e^{\lambda_{r} t}$ are linearly independent over the field of rational functions of $t$ (Lemma 41 of [6(b)]). Moreover as we have seen $\lambda_{1}^{\prime}, \cdots, \lambda_{r}^{\prime}$ are roots of the polynomial

$$
\prod_{s \in W}\left(\zeta-s \Lambda\left(H_{0}\right)\right)
$$


all whose roots are of multiplicity $N$. Therefore it follows from Lemma 27 that the degree of $p(t)=p_{1}^{\prime}(t)$ cannot exceed $N-1$. This contradiction proves our assertion.

We know from Lemma 7 that every element in $A$ can be written in the form $a \exp H$ where $a$ lies in the center of $M \cap K$ and $H \in \mathfrak{h}_{0}$. Let $\mathfrak{h}_{1}$ denote a nonempty open connected subset of $\mathfrak{h}_{0}$ such that $a \exp H \in^{\prime} A$ for all $H \in \mathfrak{h}_{1}$. Since $A^{\prime}$ is dense in $A$ (Lemma 8), we can choose an element $H_{0} \in \mathfrak{h}_{1}$ such that $a \exp H_{0}=h_{0} \in A^{\prime}$. Moreover $a$ being in the center of $M \cap K$, it is obvious that

$$
\left|d_{-}(a \exp H)\right|^{1 / 2}=\left|d_{-}(\exp H)\right|^{1 / 2}=\epsilon \Delta_{-}(H)
$$

if $H$ is sufficiently near $H_{0}$ in $\mathfrak{h}_{1}$. (Here $\epsilon= \pm 1$ and it is independent of $H$.) Hence if we apply the principle of analytic continuation to the function $\Delta_{-}(H) \tau(a \exp H)$ on $\mathfrak{h}_{1}$ we obtain the following theorem from our result above.

TheOREM 4. Let $\tau \neq \neq 0$ be an invariant analytic function on 'M. Suppose $\tau$ is an eigenfunction of the operator $\delta(z)$ for every $z \in \mathbb{Z}$. Then there exists a linear function $\Lambda$ on $\mathfrak{h}$ such that $\delta(z) \tau=\Lambda(\gamma(z)) \tau(z \in \mathcal{B})$ and $\Lambda$ is unique up to an operation of $W$. If $a$ is any point in the center of $M \cap K$ and $\mathfrak{h}_{1}$ a connected component of the set ' $\mathfrak{h}_{a}$ of all points $H \in \mathfrak{h}_{0}$ for which $d_{+}(a \exp H) \neq 0$, we can select for each $s \in W$ a polynomial function $p_{s}$ on $\mathfrak{h}$ such that

$$
\Delta_{-}(H) \tau(a \exp H)=\left|d_{+}(a \exp H)\right|^{-1 / 2} \sum_{s \in W} p_{s}(H) e^{s \Delta(H)} \quad\left(H \in \mathfrak{h}_{1}\right) .
$$

Let $W_{\Lambda}$ be the subgroup consisting of those elements $s \in W$ for which $s \Lambda=\Lambda$. Then under the assumption that $p_{s \sigma}=p_{s}\left(s \in W, \sigma \in W_{\Lambda}\right)$, the polynomial functions $p_{s}$ are uniquely determined and their degrees are smaller than the order of $W_{\Lambda}$.

The above formula is very similar to the one obtained by Weyl [14(a)] for the character of an irreducible finite-dimensional representation of $G$. Actually it is possible to improve our result in two ways. First of all we have not yet made full use of the differential equations $v \sigma=\Lambda(v) \sigma(v \in J)$ of Lemma 26 . Secondly the fact that $\tau$ defines an analytic function on ' $A$ (and not merely on $A^{\prime}$ ) allows us to obtain a little more information about $\Lambda$ and $p_{s}$. We shall however not pursue this matter here further.

10. An important special case. In this section we shall make the following two additional assumptions. (1) $\mathfrak{h}_{0} \subset \mathfrak{l}_{0}$ and (2) $G$ has a faithful finite-dimensional representation. Then $G$ can be imbedded in a complex analytic group $G_{c}$ with the Lie algebra $g$ in such a way that the (real) analytic subgroup of $G_{c}$ corresponding to $g_{0}$ coincides with $G$. In this case $K$ is compact, $A_{+}=\{1\}$ and $M=K$. Moreover if $A_{0}$ is the connected component of 1 in $A, A_{0}$ is a maximal connected abelian subgroup of $K$. But $K$ being compact, this implies [12] that $A_{0}$ is maximal abelian in $K$ and therefore $A=A_{0}$. Hence $A$ 
is connected. Moreover in this case there are no roots in the set $P_{+}$so that $P=P_{0} \cup P_{-}$.

Let $\mathfrak{u}=\mathfrak{f}_{0}+(-1)^{1 / 2} \mathfrak{p}_{0}$ and let $U$ be the (real) analytic subgroup of $G_{c}$ corresponding to $\mathfrak{u}$. Then $U$ is compact and $K=G \cap U$ (see Cartan [1(a)] and Mostow [10]). As before let $W$ denote the Weyl group of $\mathfrak{g}$ with respect to $\mathfrak{h}$. For each $s \in W$ we can choose an element $u \in U$ such that $\operatorname{Ad}(u) H=s H$ $(H \in \mathfrak{h})$ and therefore if we put $h^{s}=u h u^{-1}(h \in A), h \rightarrow h^{s}$ defines an automorphism of $A$. For any function $f$ on $A$ we denote by $f^{s}(s \in W)$ the function $h \rightarrow f\left(h^{a^{-1}}\right)(h \in A)$.

For each root $\alpha$ we have the character $\xi_{\alpha}$ of $A$ given by $\xi_{\alpha}(\exp H)=e^{\alpha(B)}$ $\left(H \in \mathfrak{h}_{0}\right)$. As before (see $\S 7$ ) let $F$ and $0 \Xi$ be the additive and the multiplicative groups generated by $\alpha$ and $\xi_{\alpha}$ respectively $(\alpha \in P)$. Then the mapping $\alpha \rightarrow \xi_{\alpha}$ $(\alpha \in P)$ can be extended (uniquely) to a homomorphism $\lambda \rightarrow \xi_{\lambda}(\lambda \in F)$ of $F$ into 0 . It is clear that if $\lambda \in F$ and $s \in W, s \lambda$ also lies in $F$. On the other hand if $\rho=2^{-1} \sum_{\alpha \in P} \alpha$ and $s_{\alpha}$ is the Weyl reflexion corresponding to the root $\alpha$, it is known (see Weyl [14(b)]) that $s_{\alpha} \rho=\rho-k \alpha$ where $k$ is an integer. Hence $s_{\alpha} \rho-\rho \in F$ and from this it follows immediately that $s \rho-\rho \in F$ for every $s \in W$. Put $\xi_{s}=\xi_{s \rho-\rho}$ and

$$
\Delta^{\prime}=\prod_{\alpha \in P}\left(1-\xi_{\alpha}^{-1}\right)
$$

Then

$$
\Delta^{\prime}(\exp H)=\prod_{\alpha \in P}\left(1-e^{-\alpha(H)}\right)=\Delta(H) e^{-\rho(H)} \quad\left(H \in \mathfrak{h}_{0}\right)
$$

if $\Delta(H)$ is defined as in $\S 8$. Therefore (see Weyl [14(b)])

$$
\Delta^{\prime}=\sum_{. \in W} \epsilon(s) \xi_{\bullet}
$$

and

$$
\left(\Delta^{\prime}\right)^{*}=\epsilon(s) \xi_{s}^{-1} \Delta^{\prime}
$$

As usual let $I^{\infty}(U)$ denote the space of invariant functions in $C^{\infty}(U)$. Also we may regard elements of $\mathscr{B}$ as differential operators on $U$. that

LEMma 29. For any $f \in C^{\infty}(A)$ there exists a unique function $\phi_{f} \in I^{\infty}(U)$ such

$$
\Delta^{\prime}(h) \phi_{f}(h)=\sum_{s \in W} \epsilon(s) \xi_{s}(h) f^{s}(h)
$$

Moreover for any $b \in \mathfrak{B}$ we can select a finite set of elements $a_{1}, \cdots, a_{r} \in \mathfrak{U}$ such that

$$
\sup \left|b \phi_{f}\right| \leqq \sup \left|a_{1} f\right|+\cdots+\sup \left|a_{r} f\right|
$$

for every $f \in C^{\infty}(A)$. 
Since $U$ is compact, $U=\bigcup_{u \in U} u A u^{-1}$ ard the uniqueness of $\phi_{f}$ is obvious. So only the existence requires proof. Let $\Theta$ denote the set of all characters of A. Put

$$
c_{\theta}(f)=\int_{A} \theta\left(h^{-1}\right) f(h) d h \quad\left(f \in C^{\infty}(A), \theta \in \Theta\right),
$$

the Haar measure $d h$ on $A$ being so normalized that $\int_{A} d h=1$. Let $\Lambda_{\theta}$ denote the linear function on $\mathfrak{h}$ such that $\theta(\exp H)=e^{\Lambda_{\theta}(H)}\left(H \in \mathfrak{h}_{0}\right)$. Then if we agree to regard a linear function on $\mathfrak{h}$ as a homomorphism of $\mathfrak{U}$ into $C$ (see $\S \S 8$ and $9)$, it is clear that $a \theta=\Lambda_{\theta}(a) \theta(a \in \mathfrak{U})$. Choose a base $H_{1}, \cdots, H_{l}$ for $\mathfrak{h}_{0}$ over $R$ such that $\exp \left(t_{1} H_{1}+\cdots+t_{l} H_{l}\right)=1$ in $A$ if and only if $t_{1}, \cdots, t_{l}$ are all integers and put $a_{0}=\left\{1-\left(4 \pi^{2}\right)^{-1}\left(H_{1}^{2}+\cdots+H_{l}^{2}\right)\right\}^{k} \in \mathfrak{U}$ where $k$ is any integer greater than $l / 2$. Then $\Lambda_{\theta}\left(H_{i}\right)=(-1)^{1 / 2} 2 \pi n_{i}$ where $n_{i}(i=1, \cdots, l)$ are integers and therefore $\Lambda_{\theta}\left(a_{0}\right)=\left(1+n_{1}^{2}+\cdots+n_{l}^{2}\right)^{k}$. Hence if $M$ denotes the sum of the convergent series

$$
\sum_{-\infty<n_{1}, \cdots, n_{l}<\infty}\left(1+n_{1}^{2}+\cdots+n_{l}^{2}\right)^{-(l+1) / 2}
$$

it is obvious that

$$
\begin{aligned}
\sum_{\theta \in \Theta}\left|c_{\theta}(f)\right| & =\sum_{\theta \in \Theta} \Lambda_{\theta}\left(a_{0}\right)^{-1}\left|\int_{A} \theta\left(h^{-1}\right) f\left(h, a_{0}\right) d h\right| \\
& \leqq M \sup \left|a_{0} f\right| \quad\left(f \in C^{\infty}(A)\right) .
\end{aligned}
$$

This proves that the series $\sum_{\theta \in \Theta} c_{\theta}(f) \theta(h)$ is absolutely convergent on $A$. Moreover as is well known, it converges to $f$ uniformly on $A$. Hence

$$
\sum_{s \in W} \epsilon(s) \xi_{s}(h) f^{s}(h)=\sum_{\theta \in \Theta} c_{\theta}(f) \sum_{s \in W} \epsilon(s) \xi_{s}(h) \theta^{s}(h) \quad(h \in A) .
$$

For every root $\alpha \in P$, put $H_{\alpha}=\left[X_{\alpha}, X_{-\alpha}\right]$. We may suppose that $X_{\alpha}, X_{-\alpha}$ are so normalized that $\alpha\left(H_{\alpha}\right)=2$. Now define $d_{\theta}$ as follows:

$$
\epsilon_{\theta} d_{\theta}=\prod_{\alpha \in P} \frac{\Lambda_{\theta}\left(H_{\alpha}\right)+\rho\left(H_{\alpha}\right)}{\rho\left(H_{\alpha}\right)} \quad(\theta \in \Theta) .
$$

Here $\epsilon_{\theta}=1$ if the right side (which is always real) is positive and -1 otherwise. Then it is known (see Weyl [14(b)]) that

$$
\sum_{s \in W} \epsilon(s) e^{s \rho(H)} e^{s \Lambda_{\theta}(H)}=\left\{\begin{array}{l}
\epsilon_{\theta} \chi_{\theta}(\exp H) \Delta(H) \text { if } d_{\theta} \neq 0, \\
0 \text { if } d_{\theta}=0
\end{array}\right.
$$

for all $H \in \mathfrak{h}_{0}$. Here $\chi_{\theta}$ is the character of an irreducible representation of $U$ of degree $d_{\theta}$. Since $\Delta^{\prime}(\exp H)=e^{-\rho(H)} \Delta(H)\left(H \in \mathfrak{h}_{0}\right)$, it follows that

$$
\sum_{s \in W} \epsilon(s) \xi_{s}(h) f^{s}(h)=\sum_{\theta \in \Theta} c_{\theta}(f) \Delta^{\prime}(h) \epsilon_{\theta} \chi_{\theta}(h)
$$


if we agree to set $\chi_{\theta}=0$ when $d_{\theta}=0$. Now consider the series

$$
\sum_{\theta \in \Theta} \epsilon_{\theta} c_{\theta}(f) \chi_{\theta}(u) \quad(u \in U)
$$

on $U$. We claim this series converges to a function in $C^{\infty}(U)$. Let $X_{1}, \cdots, X_{r}$ $\in \mathfrak{u}(r \geqq 0)$ and consider the series

$$
\sum_{\theta \in \Theta} \epsilon_{\theta} c_{\theta}(f) \chi_{\theta}\left(u, X_{1} X_{2} \cdots X_{r}\right) .
$$

For any $\theta$ for which $d_{\theta} \neq 0$ let $\sigma_{\theta}$ denote the irreducible unitary representation of $U$ on a finite-dimensional Hilbert space $V$ corresponding to the character $\chi_{\theta}$. We denote the corresponding representations of $\mathfrak{g}$ and $\mathfrak{B}$ also by $\sigma_{\theta}$. Then

$$
\left|\chi_{\theta}\left(u, X_{1} \cdots X_{r}\right)\right|=\left|\operatorname{sp}\left(\sigma_{\theta}(u) \sigma_{\theta}\left(X_{1} \cdots X_{r}\right)\right)\right| \quad(u \in U) .
$$

Now for any linear transformation $T$ on $V$, let $T^{*}$ denote the adjoint of $T$ and put $\|T\|=\left\{\operatorname{sp}\left(T T^{*}\right)\right\}^{1 / 2}$. Then $\left|\operatorname{sp}\left(T_{1} T_{2}\right)\right| \leqq\left\|T_{1}\right\|\left\|T_{2}\right\|$ for any two linear transformations $T_{1}$ and $T_{2}$ and therefore

$$
\left|\chi_{\theta}\left(u, X_{1} X_{2} \cdots X_{r}\right)\right| \leqq d_{\theta}\left\|\sigma_{\theta}\left(X_{1} \cdots X_{r}\right)\right\|
$$

since $\left\|\sigma_{\theta}(u)\right\|=d_{\theta}$. On the other hand if $X \in \mathfrak{u},-\sigma_{\theta}(X)$ is the adjoint of $\sigma_{\theta}(X)$ and therefore

$$
\left\|\sigma_{\theta}\left(X_{1} \cdots X_{r}\right)\right\|^{2}=\operatorname{sp} \sigma_{\theta}(b)
$$

where $b=(-1)^{r}\left(X_{r} X_{r-1} \cdots X_{1}\right)\left(X_{1} X_{2} \cdots X_{r}\right)$. On the other hand we can choose $z_{1} \in \mathbb{B}$ such that $b$ can be written in the form

$$
b=z_{1}+\sum_{i=1}^{q}\left[b_{i}, b_{i}^{\prime}\right]
$$

where $b_{i}, b_{i}^{\prime} \in \mathcal{B}$ (see Lemma 39 of $[6(b)]$ ). Then $\mathrm{sp} \sigma_{\theta}(b)=\operatorname{sp} \sigma_{\theta}\left(z_{1}\right)$. But in view of the above formula for $\chi_{\theta}$, it is clear that the highest weight of $\sigma_{\theta}$ must be of the form $s \Lambda_{\theta}^{\prime}-\rho$ where $s \in W$ and $\Lambda_{\theta}^{\prime}=\Lambda_{\theta}+\rho$. Therefore if $z=1+z_{1}$, it follows (see [6(b), p. 73]) that

$$
\begin{aligned}
\left\|\sigma_{\theta}\left(X_{1} \cdots X_{r}\right)\right\| & \leqq 1+\left\|\sigma_{\theta}\left(X_{1} \cdots X_{r}\right)\right\|^{2}=1+\operatorname{sp} \sigma_{\theta}\left(z_{1}\right)=1+d_{\theta} \Lambda_{\theta}^{\prime}\left(\gamma\left(z_{1}\right)\right) \\
& \leqq d_{\theta} \Lambda_{\theta}^{\prime}(\gamma(z))
\end{aligned}
$$

where $\gamma$ is defined as in $\S 8$. This shows that

$$
\left|\chi_{\theta}\left(u, X_{1} \cdots X_{r}\right)\right| \leqq d_{\theta}^{2} \Lambda_{\theta}^{\prime}((\gamma z))
$$

which remains true also if $d_{\theta}=0$ since then $\chi_{\theta}=0$. On the other hand

$$
d_{\theta}^{2}=\Lambda_{\theta}^{\prime}(a)
$$

where $a=\left\{\prod_{\alpha \in P} \rho\left(H_{\alpha}\right)\right\}^{-2} \prod_{\alpha \in P} H_{\alpha}^{2} \in \mathfrak{U}$. Therefore 


$$
\left|\chi_{\theta}\left(u, X_{1} \cdots X_{r}\right)\right| \leqq \Lambda_{\theta}^{\prime}(a \gamma(z))
$$

and

$$
\sum_{\theta \in \Theta}\left|\epsilon_{\theta} c_{\theta}(f) \chi_{\theta}\left(u, X_{1} \cdots X_{r}\right)\right| \leqq \sum_{\theta \in \Theta}\left|c_{\theta}(f) \Lambda_{\theta}^{\prime}(\operatorname{ar}(z))\right| .
$$

Let $\lambda$ denote the automorphism of $\mathfrak{U}$ over $C$ given by $\lambda(H)=H+\rho(H)(H \in \mathfrak{h})$. Then if $a^{\prime}=\lambda(a \gamma(z))$, it is clear that

$$
a^{\prime} \theta=\Lambda_{\theta}^{\prime}(a \gamma(z)) \theta \quad(\theta \in \Theta) .
$$

Hence

$$
c_{\theta}(f) \Lambda_{\theta}^{\prime}(a \gamma(z))=\int_{A} \theta\left(h, a^{\prime}\right) f\left(h^{-1}\right) d h=\int_{A} \theta\left(h^{-1}\right) f\left(h, a^{\prime}\right) d h
$$

and therefore if $a^{\prime \prime}=a_{0} a^{\prime}$,

$$
\sum_{\theta \in \Theta}\left|\epsilon_{\theta} c_{\theta}(f) \chi_{\theta}\left(u, X_{1} \cdots X_{r}\right)\right| \leqq \sum_{\theta \in \Theta}\left|\int \theta\left(h^{-1}\right) f\left(h, a^{\prime}\right) d h\right| \leqq M \sup \left|a^{\prime \prime} f\right|
$$

by our earlier result. Now if we introduce in $C^{\infty}(U)$ the topology which is usual in the theory of distributions (see Schwartz $[11$, p. 68]), it becomes a complete locally convex space and the above result shows that $\sum_{\theta \in \Theta} \epsilon_{\theta} c_{\theta}(f) \chi_{\theta}$ converges in $C^{\infty}(U)$ to some element $\phi_{f}$. This implies in particular the uniform convergence of $\sum_{\theta \in \Theta} \epsilon_{\theta} c_{\theta}(f) b \chi_{\theta}$ on $U$ to $b \phi_{f}(b \in \mathfrak{B})$. Taking $b=1$ we see that $\sum_{\theta \in \Theta} \epsilon_{\theta} C_{\theta}(f) \chi_{\theta}$ converges to $\phi_{f}$ uniformly on $U$. Therefore since the functions $\chi_{\theta}$ are all invariant, the same holds for $\phi_{f}$ and so $\phi_{f} \in I^{\infty}(U)$. Moreover

$$
\begin{aligned}
\Delta^{\prime}(h) \phi_{f}(h) & =\sum_{\theta \in \Theta} c_{\theta}(f) \epsilon_{\theta} \Delta^{\prime}(h) \chi_{\theta}(h) \\
& =\sum_{s \in \boldsymbol{W}} \epsilon(s) \xi_{s}(h) f^{s}(h)
\end{aligned}
$$

Finally it follows from the inequality obtained above that

$$
\sup \left|b \phi_{f}\right| \leqq M \sup \left|a^{\prime \prime} f\right|
$$

where $b=X_{1} X_{2} \cdots X_{r}$. Since every element in $\mathfrak{B}$ can be written as a linear combination of such elements $b$, the last statement of the lemma is now obvious.

Corollary 1. Suppose $f$ is a finite linear combination of elements in $\Theta$ and $f^{s}=f(s \in W)$. Then there exists an invariant analytic function $\phi$ on $U$ which coincides with $f$ on $A$.

For $c_{\theta}(f)$ being zero for all $\theta \in \Theta$ except a finite number,

$$
\phi_{f}=\sum_{\theta \in \Theta} c_{\theta}(f) \epsilon_{\theta} \chi_{\theta}
$$


is a finite linear combination of irreducible characters of $U$ and so it is analytic. Now since $f^{*}=f(s \in W)$,

$$
\sum_{s \in W} \epsilon(s) \xi_{s} f^{s}=f \sum_{s \in W} \epsilon(s) \xi_{s}=f \Delta^{\prime} .
$$

Hence $\Delta^{\prime}(h)\left(f(h)-\phi_{f}(h)\right)=0(h \in A)$. But since $f$ and $\phi_{f}$ are both continuous we can conclude that $f(h)-\phi_{f}(h)=0(h \in A)$.

Now introduce on $C^{\infty}(A)$ and $C^{\infty}(K)$ the topologies used in the theory of distributions (see Schwartz $\left[11\right.$, p. 66]). Obviously $I^{\infty}(K)$ is closed in $C^{\infty}(K)$.

CoRollary 2. For any $f \in C^{\infty}(A)$ there exists a unique function $F_{f} \in I^{\infty}(K)$ such that

$$
\Delta^{\prime}(h) F_{f}(h)=\sum_{s \in W} \epsilon(s) \xi_{s}(h) f^{s}(h) \quad(h \in A) .
$$

Moreover $f \rightarrow F_{f}$ is a continuous linear mapping of $C^{\infty}(A)$ into $I^{\infty}(K)$.

The uniqueness of $F_{f}$ follows from the fact that $K=\mathrm{U}_{k \in K} k A k^{-1}$. On the other hand the restriction on $K$ of the function $\phi_{f}$ of Lemma 29 obviously satisfies the required conditions for $F_{f}$. The linearity of the mapping follows from the uniqueness of $F_{f}$ and the continuity from the last statement of Lemma 29.

CoROllary 3. Suppose $f$ is a finite linear combination of elements in $\Theta$ and $f^{*}=f\left(s \in W_{-}\right)$. Then there exists an invariant analytic function on $K$ which coincides with $f$ on $A$.

Since $K$ is compact and connected, it is obvious that the method of proof of Lemma 29 is applicable to the pair $(K, A)$ instead of $(U, A)$. The required result therefore follows from the Corollary 1 above.

Now in the present case $M=K$ and $\mathfrak{q}=\mathfrak{p}$. Hence $\mathfrak{X}$ being the subalgebra of $\mathfrak{B}$ generated by $(1, \mathfrak{t}), \mathfrak{M}=\mathfrak{X}$. Let $r$ be the number of roots in $P_{0}$. Then $\operatorname{dim} \mathfrak{p}=2 r$ since $\left(X_{\alpha}, X_{-\alpha}\right)_{\alpha \in P_{0}}$ is a base for $\mathfrak{p}$ over $C$. Consider the polynomial

$$
\sum_{\beta \in P_{0}}\left\{t-\left(2-\xi_{\beta}-\xi_{\beta}^{-1}\right)\right\}=t^{r}-\sigma_{1} t^{r-1}+\cdots+(-1)^{r} \sigma_{r}
$$

in the indeterminate $t$. $\left(\sigma_{1}, \cdots, \sigma_{r}\right.$ are functions on $A$.) If $s \in W_{-}$and $\beta \in P_{0}$, it is clear that either $s \beta$ or $-s \beta$ is in $P_{0}$. Therefore it is obvious that $\sigma_{i}^{s}=\sigma_{i}$ $\left(s \in W_{-}, 1 \leqq i \leqq r\right)$. Hence from Corollary 3 to Lemma 29 we can extend $\sigma_{i}$ to an invariant analytic function on $K$. We denote this extension again by $\sigma_{i}$. Let $S(k)$ denote the restriction of $(\operatorname{Ad}(k)-I)\left(\operatorname{Ad}\left(k^{-1}\right)-I\right)(k \in K)$ on $p$. (Here $I$ is the identity mapping of g.) Then if we define $d_{+}(k)$ as in $\S 5$, we have the following result.

LEMMA 30. $\sigma_{r}(k)=d_{+}(k)$ and 


$$
(S(k))^{r}-\sigma_{1}(k)(S(k))^{r-1}+\cdots+(-1)^{r} \sigma_{r}(k) I_{\mathfrak{p}}=0 \quad(k \in K)
$$

where $I_{\mathfrak{p}}$ is the identity mapping of $\mathfrak{p}$.

Since $K=\bigcup_{k \in K} k A k^{-1}$, it is obviously enough to prove these relations on $A$. But if $h \in A$, the eigenvalues of the restriction of $\mathrm{Ad}(h)$ on $\mathfrak{p}$ are obviously $\xi_{\beta}(h)$ and $\xi_{\beta}\left(h^{-1}\right)\left(\beta \in P_{0}\right)$. Hence every eigenvalue of $S(h)$ is of the form

$$
\left(\xi_{\beta}(h)-1\right)\left(\xi_{\beta}\left(h^{-1}\right)-1\right)=2-\xi_{\beta}(h)-\xi_{\beta}(h)^{-1} \quad\left(\beta \in P_{0}\right) .
$$

Therefore if we replace $t$ by $S(h)$ in the polynomial

$$
\prod_{\beta \in P_{0}}\left\{t-\left(2-\xi_{\beta}(h)-\xi_{\beta}(h)^{-1}\right)\right\}=t^{r}-\sigma_{1}(h) t^{r-1}+\cdots+(-1)^{r} \sigma_{r}(h)
$$

we get zero (provided the last term is interpreted as $\sigma_{r}(h) I_{\mathfrak{p}}$ ). Moreover

$$
\begin{aligned}
\sigma_{r}(h) & =\prod_{\beta \in P_{0}}\left(2-\xi_{\beta}(h)-\xi_{\beta}(h)^{-1}\right) \\
& =\prod_{\beta \in P_{0}}\left(\xi_{\beta}(h)-1\right)\left(\xi_{\beta}(h)^{-1}-1\right)=d_{+}(h)
\end{aligned}
$$

and so the lemma is proved. We note that $d_{+}$is never negative on $A$ and hence also never on $K$.

Let $X_{1}, \cdots, X_{n}$. be a base for $g$ over $C$ and put $g_{i j}=B\left(X_{i}, X_{j}\right)(1 \leqq i, j \leqq n)$ (see $\$ 2$ for the definition of the bilinear form $B(X, Y)$ on $\mathfrak{g}$ ). Then since $\mathfrak{g}$ is semisimple, the matrix $\left(g_{i j}\right)_{1 \leqq i, j \leqq n}$ is nonsingular. Let $\left(g^{i j}\right)_{1 \leqq i, j \leqq n}$ denote its inverse. Put $\omega=\sum_{1 \leqq i, j \leqq n} g^{i j} X_{i} X_{j}$. Then it is well known that $\omega$ is independent of the choice of the base used in its definition and it lies in 3. In particular if $\left(X_{1}, \cdots, X_{m}\right)$ is a base for $\mathfrak{t}$ and $\left(X_{m+1}, \cdots, X_{n}\right)$ a base for $\mathfrak{p}, \omega=\omega_{K}+\omega_{+}$ where

$$
\omega_{K}=\sum_{1 \leqq i, j \leqq m} g^{i j} X_{i} X_{j}
$$

and

$$
\omega_{+}=\sum_{m<i, j \leqq n} g^{i j} X_{i} X_{j}
$$

Since $\omega^{k}=\omega(k \in K)$, it follows easily that $\omega_{K}^{k}=\omega_{K}$ and $\omega_{+}^{k}=\omega_{+}$. Hence $\omega_{K}$ lies in the center $3_{K}$ of $\mathfrak{X}$. We shall call $\omega$ and $\omega_{K}$ the Casimir operators of $\mathfrak{g}$ and

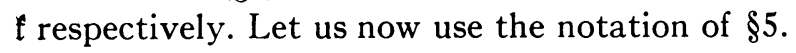

Lemma 31. There exists an analytic mapping $\gamma_{\omega}$ of $K$ into $\mathfrak{Q} \times \mathfrak{X}$ such that

$$
\Gamma_{k}\left(\gamma_{\omega}(k)\right)=d_{+}(k) \omega \quad(k \in K) .
$$

Put

$$
T(k)=(-1)^{r-1}\left\{(S(k))^{r-1}-\sigma_{1}(k)(S(k))^{r-2}+\cdots+(-1)^{r-1} \sigma_{r-1}(k) I_{\mathfrak{p}}\right\}
$$

where $S(k)$ is defined as in Lemma 30 . Then 


$$
S(k) T(k)=T(k) S(k)=d_{+}(k) I_{\mathfrak{p}}
$$

If $X_{1}, \cdots, X_{q}$ is a base for $\mathfrak{p}$, it is clear that

$$
\omega_{+}=\sum_{1 \leqq i, j \leqq q} g^{i j} X_{i} X_{j}
$$

where $\left(g^{i j}\right)_{1 \leqq i, j \leqq q}$ is the inverse of the matrix $g_{i j}=B\left(X_{i}, X_{j}\right) 1 \leqq i, j \leqq q$. Now $k$ being a fixed element in $K$, suppose $S^{\prime}, T^{\prime}$ are two linear transformations on p which commute with the restriction of Ad (k) on p. Put $Y_{i}=S^{\prime} X_{i}, Z_{i}=T^{\prime} X_{i}$, $X^{i}=\sum_{j=1}^{g} g^{i j} X_{j}, Y^{i}=S^{\prime} X^{i}$ and $Z^{i}=T^{\prime} X^{i}(1 \leqq i \leqq q)$. It is obvious that

$$
X=\sum_{i=1}^{q} B\left(X, X_{i}\right) X^{i}
$$

for any $X \in \mathfrak{p}$ and therefore

$$
\operatorname{Ad}(k) X=\sum_{i=1}^{q} B\left(\operatorname{Ad}(k) X, X_{i}\right) X^{i}=\sum_{i=1}^{q} B\left(X, \operatorname{Ad}\left(k^{-1}\right) X_{i}\right) X^{i} .
$$

Hence

$$
\operatorname{Ad}(k) Y_{i}=S^{\prime} \operatorname{Ad}(k) X_{i}=\sum_{j=1}^{q} B\left(X_{i}, \operatorname{Ad}\left(k^{-1}\right) X_{j}\right) S^{\prime} X^{j}
$$

and

$$
\begin{aligned}
\sum_{k=1}^{q}\left(\operatorname{Ad}(k) Y_{i}\right) Z^{i} & =\sum_{i, j}\left(S^{\prime} X^{j}\right)\left(T^{\prime} X^{i}\right) B\left(X_{i}, \operatorname{Ad}\left(k^{-1}\right) X_{i}\right) \\
& =\sum_{i}\left(S^{\prime} X^{j}\right)\left(T^{\prime} \operatorname{Ad}\left(k^{-1}\right) X_{j}\right)=\sum_{j} Y^{i}\left(\operatorname{Ad}\left(k^{-1}\right) Z_{i}\right) .
\end{aligned}
$$

This proves that

$$
\sum_{1 \leqq i, j \leqq q} g^{i j}\left(\operatorname{Ad}(k) Y_{i}\right) Z_{j}=\sum_{1 \leqq i, j \leqq q} g^{i j} Y_{i}\left(\operatorname{Ad}\left(k^{-1}\right) Z_{j}\right)
$$

and therefore

$$
\sum_{1 \leqq i, j \leqq q} g^{i j}\left[\operatorname{Ad}(k) Y_{i}, Z_{j}\right]=\sum_{1 \leqq i, j \leqq q} g^{i j}\left[Y_{i}, \operatorname{Ad}\left(k^{-1}\right) Z_{j}\right] .
$$

Now put $T^{\prime}(k)=$ Ad $\left(k^{-1}\right) T(k)-T(k), X_{j}(k)=T(k) X_{j}$ and $X_{j}^{\prime}(k)=T^{\prime}(k) X_{j}$ $1 \leqq j \leqq q$. Then

$$
\begin{aligned}
\sum_{1 \leqq i, j \leq q} g^{i j} \Gamma_{k}\left(X_{i} X_{i}(k) \times 1\right) \\
=\sum_{i, j} g^{i j}\left(\operatorname{Ad}\left(k^{-1}\right) X_{i}-X_{i}\right)\left(T^{\prime}(k) X_{j}\right)+\sum_{i, j} g^{i j}\left[X_{i}, T^{\prime}(k) X_{i}\right] \\
=\sum_{i, j} g^{i j} X_{i}\left\{(\operatorname{Ad}(k)-I) T^{\prime}(k) X_{i}\right\}+\sum_{i, j} g^{i j}\left[X_{i}, T^{\prime}(k) X_{j}\right] .
\end{aligned}
$$

But $(\operatorname{Ad}(k)-I) T^{\prime}(k)=S(k) T(k)=d_{+}(k) I_{\mathfrak{p}}$. So if we put 


$$
b(k)=\frac{1}{2} \sum_{1 \leqq i, j \leqq q} g^{i j}\left(X_{i} X_{j}(k)+X_{j}(k) X_{i}\right),
$$

it is clear that

$$
\Gamma_{k}(b(k) \times 1)=d_{+}(k) \omega_{+}+\sum_{i, j} g^{i j}\left[X_{i}, X_{j}^{\prime}(k)\right]-\frac{1}{2} \sum_{i, j} g^{i j} \Gamma_{k}\left(\left[X_{i}, X_{j}(k)\right] \times 1\right) .
$$

Put $Y(k)=\sum_{i, j} g^{i j}\left[X_{i}, X_{j}(k)\right]$ and $Y^{\prime}(k)=\sum_{i, j} g^{i j}\left[X_{i}, X_{j}^{\prime}(k)\right]$. It follows from our remarks above that $\Gamma_{k}(Y(k) \times 1)=\operatorname{Ad}\left(k^{-1}\right) Y(k)-Y(k)=0$. Moreover $Y^{\prime}(k) \in[\mathfrak{p}, \mathfrak{p}] \subset \mathfrak{l}$. Hence if we set

$$
\gamma_{\omega}(k)=b(k) \times 1+d_{+}(k)\left(1 \times \omega_{K}\right)-1 \times Y^{\prime}(k),
$$

$\Gamma_{k}\left(\gamma_{\omega}(k)\right)=d_{+}(k) \omega$. This proves the lemma.

On the other hand ' $K$ being the open submanifold of $K$ consisting of those points $k$ for which $d_{+}(k) \neq 0$, we have a differential operator $\delta(\omega)$ defined on ' $K$ (see $\S 5)$. It follows from the definition of $\delta(\omega)$ that

$$
d_{+}(k) \delta_{k}(\omega)=d_{+}(k) \omega_{K}-Y^{\prime}(k) \quad\left(k \in{ }^{\prime} K\right) .
$$

This means that there exists an analytic differential operator $D$ on $K$ which coincides with $d_{+} \delta(\omega)$ on ' $K$.

Lemma 32. Let $D^{*}$ denote the adjoint of $D$. Then if $f \in I^{\infty}(K), D f=D^{*} f$.

In view of the corollary to Lemma 8 , it is enough to show that $D f$ and $D^{*} f$ coincide on $K^{\prime}=K \cap G^{\prime}$. Hence, as we have seen during the proof of Theorem 1 , it is sufficient to prove that

$$
\int_{K} g(k) f(k, D) d k=\int_{K} g(k, D) f(k) d k
$$

for every $g \in I_{c}^{\infty}\left(K^{\prime}\right)$. (Here $d k$ is the Haar measure on $K$.) But since $D$ is an invariant operator, $D f$ and $D g$ are invariant functions. Hence (see Weyl $[14(\mathrm{a})])$

$$
\int_{\boldsymbol{K}} g(k) f(k, D) d k=\int_{\boldsymbol{A}}\left|d_{-}(h)\right| \boldsymbol{g}(h) f(h, D) d h
$$

and

$$
\int_{\boldsymbol{K}} g(k, D) f(k) d k=\int_{\boldsymbol{A}}\left|d_{-}(h)\right| g(h, D) f(h) d h
$$

if $d k$ is suitably normalized. On the other hand since $d_{+}=\left|d_{+}\right|$, we can conclude from Theorem 2 and the corollary to Lemma 25 that if $h \in A^{\prime}$,

$$
\left|d_{-}(h)\right| f(h, D)=f\left(h, D^{\prime}\right)
$$

where $D^{\prime}$ denotes the differential operator $|d|^{1 / 2} \gamma(\omega) \circ|d|^{1 / 2}$ on $A^{\prime}$. Moreover 
$(\gamma(\omega))^{*}=\gamma\left(\omega^{*}\right)=\gamma(\omega)$ from Lemma 20 and so $D^{\prime}$ coincides with its adjoint on $A^{\prime}$. Hence

$$
\begin{aligned}
\int_{K} g(k) f(k, D) d k & =\int_{\boldsymbol{A}} g(h) f\left(h, D^{\prime}\right) d h=\int_{\boldsymbol{A}} g\left(h, D^{\prime}\right) f(h) d h \\
& =\int_{\boldsymbol{K}} g(k, D) f(k) d k .
\end{aligned}
$$

Let $\gamma^{\prime}(\omega)$ denote the image of $\gamma(\omega)$ under the automorphism of $\mathfrak{u}$ which maps $H$ on $H+\rho(H)(H \in \mathfrak{h})$.

Lemma 33. Define the mapping $f \rightarrow F_{f}$ of $C^{\infty}(A)$ into $I^{\infty}(K)$ as in Corollary 2 to Lemma 29. Then

$$
D F_{f}=d_{+} F_{\gamma^{\prime}(\omega) f}
$$$$
\left(f \in C^{\infty}(A)\right) \text {. }
$$

It would be sufficient to prove that $D F_{f}$ and $d_{+} F_{\gamma^{\prime}(\omega) f}$ coincide on $A^{\prime}$. Let $g$ denote the restriction of $F_{f}$ on $A^{\prime}$. Then

$$
g(h)=\Delta^{\prime}(h)^{-1} \sum_{s \in W} \epsilon(s) \xi_{s}(h) f^{s}(h)
$$

and it follows from Theorem 2 and the corollary to Lemma 25 that $D F_{f}$ coincides on $A^{\prime}$ with $D^{\prime} g$ where $D^{\prime}=d_{+}|d|^{-1 / 2} \gamma(\omega) \circ|d|^{1 / 2}$. Let $H_{0}$ be any point in $\mathfrak{h}_{0}$ such that $h_{0}=\exp H_{0} \in A^{\prime}$ and let $V$ be an open connected neighborhood of $H_{0}$ in $\mathfrak{h}_{0}$ such that $\exp H \in A^{\prime}$ for all $H \in V$. Put

$$
g^{\prime}(H)=\sum_{s \in W} \epsilon(s) e^{s \rho(H)} f^{\prime}(\exp H)
$$

Then $g(\exp H) \Delta(H)=g^{\prime}(H)(H \in V)$ where $\Delta(H)$ is defined as in $\S 8$. Hence it is obvious that

$$
g\left(\exp H, D^{\prime}\right)=d_{+}(\exp H) \Delta(H)^{-1} g^{\prime}(H, \gamma(\omega))
$$

if we regard $\gamma(\omega)$ as a differential operator on $V$. On the other hand if $f_{z}^{\prime}(H)$ $=e^{\operatorname{s\rho }(H)} f^{\natural}(\exp H)\left(H \in \mathfrak{h}_{0}\right)$ it is clear that

$$
f_{s}^{\prime}(H, \gamma(\omega))=f_{1}^{\prime}\left(s^{-1} H, \gamma(\omega)\right)
$$

since $\gamma(\omega)$ is an invariant of $W$. Furthermore

$$
f_{1}^{\prime}(H, \gamma(\omega))=e^{\rho(H)} f^{\prime}\left(H, \gamma^{\prime}(\omega)\right)
$$

where $f^{\prime}(H)=f(\exp H)$. Hence

$$
\begin{aligned}
g^{\prime}(H, \gamma(\omega)) & =\sum_{\bullet \in W} \epsilon(s) e^{\rho \rho(H)} f^{\prime}\left(s^{-1} H, \gamma^{\prime}(\omega)\right) \\
& =\sum_{. \in W} \epsilon(s) e^{\alpha \rho(H)} f_{0}^{*}(\exp H)
\end{aligned}
$$

where $f_{0}=\boldsymbol{\gamma}^{\prime}(\boldsymbol{\omega}) f$ and therefore 


$$
g\left(\exp H, D^{\prime}\right)=d_{+}(\exp H) F_{\gamma^{\prime}(\omega) f}(\exp H)
$$

This proves that $D F_{f}$ coincides with $d_{+} F_{\gamma^{\prime}(\omega) f}$ on some neighborhood of $h_{0}$ in $A^{\prime}$. Since $h_{0}$ is an arbitrary point of $A^{\prime}$ our assertion follows.

Let $\tau$ be a distribution on $K$. Then by Corollary 2 to Lemma 29, the mapping $\Phi: f \rightarrow \tau\left(d_{+} F_{f}\right)\left(f \in C^{\infty}(A)\right)$ is a distribution on $A$. The following theorem contains the main result of the present section.

TheOREM 5. Suppose there exists a complex number $c$ such that $D \tau=c d_{+} \tau$. Then $\Phi$ coincides with an analytic function on $A$.

If $f \in C^{\infty}(A), D \tau\left(F_{f}\right)=\tau\left(D F_{f}\right)=\Phi\left(\gamma^{\prime}(\omega) f\right)$ from Lemmas 32 and 33. But $D \tau\left(F_{f}\right)=c \tau\left(d_{+} F_{f}\right)=c \Phi(f)$. Hence

$$
\Phi\left(\gamma^{\prime}(\omega) f\right)=c \Phi(f) \quad\left(f \in C^{\infty}(A)\right) .
$$

Now let $H_{1}, \cdots, H_{l}$ be a base for $\mathfrak{h}_{0}$ over $R$ such that $B\left(H_{i}, H_{j}\right)=-\delta_{i j}$ $1 \leqq i, j \leqq l$. Then if we normalize $X_{\alpha}, X_{-\alpha}(\alpha \in P)$ in such a way that $B\left(X_{\alpha}\right.$, $\left.X_{-\alpha}\right)=1$, it is clear that

$$
\omega=-\left(H_{1}^{2}+\cdots+H_{l}^{2}\right)+\sum_{\alpha \in P}\left(X_{\alpha} X_{-\alpha}+X_{-\alpha} X_{\alpha}\right) .
$$

Hence

$$
\omega \equiv-\left(H_{1}^{2}+\cdots+H_{l}^{2}\right)+\sum_{\alpha \in P} H_{\alpha} \bmod \sum_{\alpha \in P} \mathfrak{B} X_{\alpha}
$$

where $H_{\alpha}=\left[X_{\alpha}, X_{-\alpha}\right]$. This proves (see Lemma 18) that

$$
\gamma^{\prime}(\omega)=-\left(H_{1}^{2}+\cdots+H_{l}^{2}\right)+2 H_{\rho}
$$

where $H_{\rho}=2^{-1} \sum_{\alpha \in P} H_{\alpha}$ is characterized by the property that $B\left(H_{\rho}, H\right)$ $=\rho(H)(H \in \mathfrak{h})$. Put

$$
\square=H_{1}^{2}+\cdots+H_{l}^{2}+2 H_{\rho} .
$$

Then it is clear that $\Phi$ satisfies the differential equation

$$
(\square+c) \Phi=0 .
$$

Since the operator $\square$ is obviously of the elliptic type (see Gårding [3]) it follows $[11 ; 8]$ that $\Phi$ is an analytic function on $A$.

We shall give an application of this theorem in $\$ 12$.

11. Application to representations. We shall now apply the preceding theory to the problem of determining the characters of irreducible representations of $G$. Let $\pi$ be a representation of $G$ on a Hilbert space $\mathfrak{S}$ and let $V$ be the Gårding subspace of $\mathfrak{S}$ (see [6(c), p. 201]). We denote by $\Omega$ the set of all equivalence classes of finite-dimensional irreducible representations of $K$ and by $\mathfrak{S}_{\mathfrak{D}}(\mathfrak{D} \in \Omega)$ the subspace consisting of those elements in $\mathfrak{S}$ which 
transform under $\pi(K)$ according to $\mathfrak{D}$. Let $\pi_{0}$ denote the renresentation of $\mathfrak{B}$ on $V$ corresponding to $\pi\left[6(\mathrm{c})\right.$, p. 201] and $I, I_{0}$ the identity mappings of $\mathfrak{E}$ and $V$ respectively. We shall say that $\pi$ is quasi-simple if there exist $\left({ }^{10}\right)$

(1) a homomorphism $\eta_{\pi}$ of $Z$ into the multiplicative group of complex numbers such that $\pi(\zeta)=\eta_{\pi}(\zeta) I(\zeta \in Z)$,

(2) a homomorphism $\chi_{\pi}$ of $\mathcal{Z}$ into $C$ such that $\pi_{0}(z)=\chi_{\pi}(z) I_{0}(z \in \mathcal{Z})$, and

(3) an integer $N$ such that $\operatorname{dim} \mathfrak{W}_{\mathfrak{D}} \leqq N d(\mathfrak{D})^{2}$ for all $\mathfrak{D} \in \Omega$. $(d(\mathfrak{D})$ is the degree of any representation in $\mathfrak{D}$.)

$\eta_{\pi}$ and $\chi_{\pi}$ respectively are called the central and the infinitesimal characters of $\pi$. Let $Q$ be a bounded linear transformation on $\mathfrak{W}$. We say that $Q$ is summable if there exists a complete orthonormal set $\left(\psi_{j}\right)_{j \in J}$ in $\mathfrak{S}$ and a regular operator $A$ such that $\left.{ }^{11}\right) \sum_{i, j \in J}\left|\left(\psi_{i}, A Q A^{-1} \psi_{j}\right)\right|<\infty$. One knows [6(d), Lemma 1] that if $Q$ is summable and $A, B$ are bounded linear operators $A Q B$ is of the trace class and sp $A Q B=\operatorname{sp} B A Q=\operatorname{sp} Q B A$. Hence in particular if $A$ is regular sp $A Q A^{-1}=\operatorname{sp} Q$. Now $\pi$ being quasi-simple, consider the operator

$$
Q_{f}=\int_{G} f(x) \pi(x) d x
$$

where $d x$ is the Haar measure on $G$ and $f \in C_{c}^{\infty}(G)$. We know [6(e), \$2] that $Q_{f}$ is summable and the mapping $T_{x}: f \rightarrow \operatorname{sp} Q_{f}\left(f \in C_{c}^{\infty}(G)\right)$ is a distribution on $G$. $T_{\pi}$ is called the character of $\pi$.

Let ' $G$ be the set of quasi-regular elements in $G$ (see $\$ 3$ ). In view of the corollary to Lemma 12, ' $G$ may be considered as an open submanifold of $G$. Our next object is to prove the following theorem.

THEOREM 6. Let $\pi$ be a quasi-simple representation of $G$ on $\mathfrak{S}$ and let $T_{\pi}$ be the character of $\pi$. Then $T_{x}$ coincides with an analytic function on ' $G$.

Since $T_{\pi}$ is an invariant distribution on $G$ (see [6(d)]), it coincides in the neighborhood of a point $x \in \in^{\prime} G$ with an analytic function if and only if the same property holds at $y x y^{-1}(y \in G)$. Hence, in view of Lemma 11 , it is enough to prove this statement when $x \in^{\prime} A=A \cap \cap^{\prime} G$ in the notation of $\$ 5$. Let $d m$ denote the Haar measure on $M$.

Lemma 34. If $f \in C_{c}^{\infty}\left(G \times{ }^{\prime} M\right)$ the operator

$$
\int_{G \times{ }^{\circ} M} f(x, m) \pi\left(x m x^{-1}\right) d x d m
$$

is summable. Moreover if $S_{\pi}(f)$ denotes its trace, the mapping

(10) Actually these conditions can be relaxed considerably without affecting our subsequent arguments. But it is hardly worthwhile to do so since they are already weak enough to be satisfied in all cases which are of interest to us.

(ii) As usual $(\phi, \psi)$ denotes the scalar product of two elements $\phi$ and $\psi$ in $\mathfrak{S}$ and $|\phi|$ the norm of $\phi$. 


$$
S_{\pi}: f \rightarrow S_{\pi}(f)
$$

is a distribution on $G \times{ }^{\prime} M$.

We can regard $G \times M$ as a Lie group and $\mathfrak{B} \times \mathfrak{M}$ the enveloping algebra of the complexification of its Lie algebra. Then $G \times{ }^{\prime} M$ is an open invariant subset of $G \times M$. For any $b \in \mathfrak{B}$, let $D^{\prime}(b)$ denote the differential operator on $G \times{ }^{\prime} M$ defined as follows. The local expression $\left(D^{\prime}(b)\right)_{x, m}$ of $D^{\prime}(b)$ at $(x, m)$ $\in G \times{ }^{\prime} M$ is given by

$$
\left(D^{\prime}(b)\right)_{x, m}=d_{+}(m)^{-r} \gamma_{b}(m)
$$

in the notation of Lemma 16. Since $m \rightarrow \gamma_{b}(m)$ is an analytic mapping, it is clear that $D^{\prime}(b)$ is in fact an analytic differential operator on $G \times{ }^{\prime} M$. On the other hand the mapping $b \rightarrow D^{\prime}(b)$ is linear and for any given $b \in \mathfrak{B}$, we can find a finite set of linearly independent elements $b_{1}, \cdots, b_{N} \in \mathfrak{B}$ such that

$$
\operatorname{Ad}\left(x^{-1}\right) b=a_{1}(x) b_{1}+\cdots+a_{N}(x) b_{N}
$$

where $a_{1}, \cdots, a_{N}$ are analytic functions on $G$. Hence

$$
\left(D^{\prime}\left(b^{x^{-1}}\right)\right)_{x, m}=\sum_{i=1}^{N} a_{i}(x)\left(D^{\prime}\left(b_{i}\right)\right)_{x, m}
$$

and therefore there exists an analytic differential operator $D(b)$ on $G \times{ }^{\prime} M$ such that $(D(b))_{x, m}=\left(D^{\prime}\left(b^{x^{-1}}\right)\right)_{x, m}\left(x \in G, m \in \in^{\prime} M\right)$. The operator $D(b)$ has the following significance. $\phi$ being any function in $C^{\infty}(G)$, consider the integral

$$
\int f(x, m) \phi\left(x m x^{-1}\right) d x d m \quad\left(f \in C_{c}^{\infty}\left(G \times{ }^{\prime} M\right)\right) .
$$

Put $\Phi(x, m)=\phi\left(x m x^{-1}\right)\left(x \in G, m \in \in^{\prime} M\right)$. Then, as we have seen in $\S 5$,

$$
\Phi(x, b ; m, v)=\phi\left(x m x^{-1}, \operatorname{Ad}(x)\left(\Gamma_{m}(b \times v)\right)\right.
$$

for $b \in \mathfrak{B}$ and $\nu \in \mathfrak{M}$. Hence it is obvious that

$$
\Phi\left(x, m, D_{x, m}(b)\right)=\phi\left(x m x^{-1}, b\right)
$$

and therefore

$$
\int f(x, m) \phi\left(x m x^{-1}, b\right) d x d m=\int f\left(x, m,(D(b))^{*}\right) \phi\left(x m x^{-1}\right) d x d m
$$

where $(D(b)) *$ is the adjoint of $D(b)$.

Now in order to prove the summability of the operator

$$
Q_{f}=\int f(x, m) \pi\left(x m x^{-1}\right) d x d m \quad\left(f \in C_{0}^{\infty}\left(G \times{ }^{\prime} M\right)\right)
$$


we proceed as follows. We can choose a homomorphism $\eta$ of $K$ into $C$ such that $\eta$ coincides with $\eta_{\pi}$ on a subgroup $Z_{0}$ of $Z$ of finite index (see [6(d), p. 239]). Then the factor group $\vec{K}=K / Z_{0}$ is compact and we can define a representation $\bar{\pi}$ of $\bar{K}$ on $\mathfrak{S}$ by setting

$$
\pi(\bar{u})=\eta\left(u^{-1}\right) \pi(u)
$$

where $u \rightarrow \bar{u}$ is the natural mapping of $K$ on $\bar{K}$. Since $\bar{K}$ is compact, $\bar{\pi}$ is equivalent to a unitary representation. Hence in view of our definition of summability, it is sufficient to consider the case when $\bar{\pi}$ is unitary and therefore the spaces $\mathfrak{S}_{\mathfrak{D}}(\mathfrak{D} \in \Omega)$ are mutually orthogonal (see [6(d), p. 244]). For any $\phi \in C^{\infty}(G)$ define $\phi(b, x)=\phi\left(x, \operatorname{Ad}\left(x^{-1}\right) b\right)(x \in G, b \in \mathfrak{B})$ and let $\Omega_{x}$ be the set of those $\mathfrak{D} \in \Omega$ for which $\mathfrak{S}_{\mathbb{D}} \neq\{0\}$. Then the following facts are known (see the proof of Lemma 3 of [6(d)]). If $z$ is an element lying in the center $\mathfrak{Z}_{k}$ of $\mathfrak{X}$, there exist, for each $\mathfrak{D} \in \Omega_{\pi}$, two complex numbers $n_{1}(\mathfrak{D}, z), n_{2}(\mathfrak{D}, Z)$ with the following property. If $\psi_{1} \in \mathfrak{S}_{\mathfrak{D}_{1}}, \psi_{2} \in \mathfrak{W}_{\mathfrak{D}_{2}}\left(\mathfrak{D}_{1}, \mathfrak{D}_{2} \in \Omega_{\pi}\right)$ and $\phi(x)$ $=\left(\psi_{1}, \pi(x) \psi_{2}\right)(x \in G)$, then

$$
\phi(z, x)=n_{1}\left(\mathfrak{D}_{1}, z\right) \phi(x), \quad \phi(x, z)=\phi(x) n_{2}\left(\mathfrak{D}_{2}, z\right) \quad(x \in G) .
$$

Moreover it is possible to choose $z_{1}, z_{2} \in \mathfrak{Z}_{K}$ such that the numbers $n_{1}\left(\mathfrak{D}, z_{1}\right)$, $n_{2}\left(\mathfrak{D}, z_{2}\right)\left(\mathfrak{D} \in \Omega_{\pi}\right)$ are all real and positive and

$$
\sum_{\mathfrak{D} \in \Omega_{\pi}} \frac{d(\mathfrak{D})^{2}}{n_{1}\left(\mathfrak{D}, z_{1}\right)}<\infty, \quad \sum_{\mathfrak{D} \in \Omega_{\pi}} \frac{d(\mathfrak{D})^{2}}{n_{2}\left(\mathfrak{D}, z_{2}\right)}<\infty .
$$

Now let $\left(\psi_{i}\right)_{i \in J}$ be a complete orthonormal base for $\mathfrak{S}$ which is composed of bases for the various $\mathfrak{S}_{\mathfrak{D}}\left(\mathfrak{D} \in \Omega_{\pi}\right)$. Let $J(\mathfrak{D})$ denote the set of those $j \in J$ for which $\psi_{j} \in \mathfrak{S D}_{\mathbb{D}}$. Then

$$
\sum_{i, j \in J}\left|\left(\psi_{i}, Q_{f} \psi_{j}\right)\right|=\sum_{i, j \in J}\left|\int f(x, m) \phi_{i j}\left(x m x^{-1}\right) d x d m\right|
$$

where $\phi_{i j}(x)=\left(\psi_{i}, \pi(x) \psi_{j}\right)(x \in G)$. On the other hand if $i \in J\left(\mathfrak{D}_{1}\right)$ and $j \in J\left(\mathfrak{D}_{2}\right)$,

$$
\phi_{i j}\left(x,\left(\operatorname{Ad}\left(x^{-1}\right) z_{1}\right) z_{2}\right)=\phi_{i j}\left(z_{1}, x, z_{2}\right)=n_{1}\left(\mathfrak{D}_{1}, z_{1}\right) n_{2}\left(\mathfrak{D}_{2}, z_{2}\right) \phi_{i j}(x) .
$$

Moreover we can find elements $b_{1}, \cdots, b_{r} \in \mathfrak{B}$ such that

$$
\left(\operatorname{Ad}\left(x^{-1}\right) z_{1}\right) z_{2}=a_{1}(x) b_{1}+\cdots+a_{r}(x) b_{r} \quad(x \in G)
$$

where $a_{1}, \cdots, a_{r}$ are analytic functions on $G$. Therefore in view of what we have seen above, it is obvious that there exists a differential operator $D$ on $G \times{ }^{\prime} M$ such that

$$
\int f(x, m, D) \phi\left(x m x^{-1}\right) d x d m=\int f(x, m) \phi\left(x m x^{-1},\left(\operatorname{Ad}\left(x m^{-1} x^{-1}\right) z_{1}\right) z_{2}\right) d x d m
$$

for all $f \in C_{c}^{\infty}\left(G \times{ }^{\prime} M\right)$ and $\phi \in C^{\infty}(G)$. On the other hand, we can find compact 
sets $\omega_{1}, \omega_{2}$ in $G$ and ' $M$ respectively such that a given $f$ is zero outside $\omega_{1} \times \omega_{2}$. Let $\omega$ be the image of $\omega_{1} \times \omega_{2}$ in $G$ under the mapping $(x, m) \rightarrow x m x^{-1}$. Then $\omega$ is compact and there exists a number $\mu$ such that $|\pi(x)| \leqq \mu$ for $x \in \omega$. Then it is clear that

$$
\left|\int f(x, m, D) \phi_{i j}\left(x m x^{-1}\right) d x d m\right| \leqq \mu \int|f(x, m, D)| d x d m
$$

and therefore

$$
\sum_{i, j \in J}\left|\left(\psi_{i}, Q_{f} \psi_{j}\right)\right| \leqq \mu N^{2} \int|f(x, m, D)| d x d m \sum_{\mathfrak{D}_{1}, \mathfrak{D}_{2} \in \Omega_{\pi}} \frac{d\left(\mathfrak{D}_{1}\right)^{2}}{n_{1}\left(\mathfrak{D}_{1}, z_{1}\right)} \frac{d\left(\mathfrak{D}_{2}\right)^{2}}{n_{2}\left(\mathfrak{D}_{2}, z_{2}\right)}
$$

since $\operatorname{dim} \mathfrak{S D D} \leqq N d(\mathfrak{D})^{2}\left(\mathfrak{D} \in \Omega_{\pi}\right)$. The series on the right being convergent, this proves that $Q_{f}$ is summable. Moreover the above result shows that for any compact set $\omega^{\prime}$ in $G \times{ }^{\prime} M$, there exists a real number $\mu^{\prime}$ such that

$$
\left|\operatorname{sp} Q_{f}\right| \leqq \mu^{\prime} \int|f(x, m, D)| d x d m
$$

where $f$ is any function in $C_{c}^{\infty}\left(G \times{ }^{\prime} M\right)$ which vanishes outside $\omega^{\prime}$. Therefore the mapping $f \rightarrow \operatorname{sp} Q_{f}$ is a distribution and the lemma is proved.

Now suppose $\alpha \in C_{c}^{\infty}(G)$ and $\beta \in C_{c}^{\infty}\left({ }^{\prime} M\right)$. We denote by $\alpha \times \beta$ the function $f$ in $C_{c}^{\infty}\left(G X^{\prime} M\right)$ given by $f(x, m)=\alpha(x) \beta(m)\left(x \in G, m \in \in^{\prime} M\right)$.

Lemma 35. There exists a distribution $\tau_{\pi}$ on ' $M$ such that

$$
S_{\pi}(\alpha \times \beta)=\left(\int_{G} \alpha(x) d x\right) \tau_{\pi}(\beta) \quad\left(\alpha \in C_{c}^{\infty}(G), \beta \in C_{c}^{\infty}\left({ }^{\prime} M\right)\right) .
$$

Let $\lambda(y) \alpha(y \in G)$ denote the function $x \rightarrow \alpha\left(y^{-1} x\right)(x \in G)$. Similarly if $\sigma$ is a distribution on $G$, we denote by $\lambda(y) \sigma$ the distribution $\alpha \rightarrow \sigma\left(\lambda\left(y^{-1}\right) \alpha\right)$ $\left(\alpha \in C_{c}^{\infty}(G)\right)$. Let $\beta$ be a fixed element in $C_{c}^{\infty}\left({ }^{\prime} M\right)$. Then it is obvious that the mapping

$$
\sigma_{\beta}: \alpha \rightarrow S_{\pi}(\alpha \times \beta)
$$

is a distribution on $G$. Moreover if

$$
Q_{\alpha}=\int \alpha(x) \beta(m) \pi\left(x m x^{-1}\right) d x d m,
$$

it is clear that

$$
Q_{\lambda(y) \alpha}=\pi(y) Q_{\alpha} \pi\left(y^{-1}\right) .
$$

Therefore since $Q_{a}$ is summable,

$$
\sigma_{\beta}(\alpha)=\operatorname{sp} Q_{\alpha}=\operatorname{sp} Q_{\lambda(y) \alpha}=\sigma_{\beta}(\lambda(y) \alpha) .
$$


This shows that $\lambda(y) \sigma_{\beta}=\sigma_{\beta}(y \in G)$. On the other hand we have the following lemma.

Lemma 36. Let $\sigma$ be a distribution on $G$ such that $\lambda(x) \sigma=\sigma$ for all $x \in G$. Then there exists a complex number $c$ such that

$$
\sigma(\alpha)=c \int_{G} \alpha(x) d x
$$

for every $\alpha \in C_{c}^{\infty}(G)$.

Assuming this for a moment, we can prove Lemma 35 as follows. Put $I(\alpha)=\int_{G} \alpha(x) d x\left(\alpha \in C_{c}^{\infty}(G)\right)$ and select a function $\alpha_{0}$ in $C_{c}^{\infty}(G)$ for which $I\left(\alpha_{0}\right)=1$. Then it follows from Lemma 36 that

$$
\sigma_{\beta}(\alpha)=\sigma_{\beta}\left(\alpha_{0}\right) I(\alpha) \quad\left(\alpha \in C_{c}^{\infty}(G)\right) .
$$

But the mapping $\beta \rightarrow \sigma_{\beta}\left(\alpha_{0}\right)=S_{\pi}\left(\alpha_{0} \times \beta\right)$ is obviously a distribution on ' $M$. Hence if we denote it by $\tau_{x}$,

$$
S_{\pi}(\alpha \times \beta)=I(\alpha) \tau_{\pi}(\beta)
$$

which proves our assertion.

Now in order to prove Lemma 36, we make use of the theory of rightinvariant differential forms on $G$ (see Chevalley [2]). For each $X \in g_{0}$, we denote by $X^{\prime}$ the right-invariant differential operator defined by

$$
\alpha\left(x, X^{\prime}\right)=\left\{\frac{d}{d t} \alpha(\exp (-t X) x)\right\}_{t=0} \quad\left(x \in G, \alpha \in C^{\infty}(G)\right) .
$$

$n$ being the dimension of $G$, let $\omega$ denote the invariant differential form of degree $n$ corresponding to the Haar measure $d x$. Then

$$
\int_{G} \alpha \omega=I(\alpha) \quad\left(\alpha \in C_{c}^{\infty}(G)\right) .
$$

Now suppose $I(\alpha)=0$. Then by the de Rham Theorem for forms with compact support, there exists a differential form $\zeta$ of degree $n-1$ and class $C^{\infty}$ with compact support such that $\alpha \omega=d \zeta$. Let $\omega_{1}, \cdots, \omega_{n}$ be a base for rightinvariant forms of degree 1 . We may assume that $\omega=\omega_{1} \wedge \omega_{2} \wedge \cdots \wedge \omega_{n}$ where $\wedge$ denotes exterior product. Let $\left(X_{1}, \cdots, X_{n}\right)$ be a base for $g_{0}$ over $R$ such that $\omega_{i}\left(X_{j}^{\prime}\right)=\delta_{i j}(1 \leqq i, j \leqq n)$. Then

$$
\zeta=\sum_{i=1}^{n} \zeta_{i} \Omega_{i}
$$

where $\zeta_{i} \in C_{c}^{\infty}(G)$ and $\Omega_{i}=\omega_{1} \wedge \omega_{2} \wedge \cdots \wedge \hat{\omega}_{i} \wedge \cdots \wedge \omega_{n}$, the circumflex signifying the deletion of the factor $\omega_{i}$ from the product. Then 


$$
\alpha \omega=d \zeta=\sum_{i=1}^{n} d \zeta_{i} \wedge \Omega_{i}+\sum_{i=1}^{n} \zeta_{i} d \Omega_{i}
$$

But $d \Omega_{i}=0$ for otherwise $\omega$ would be the derivative of a right-invariant differential form and this is known to be impossible (see Koszul [9, Theorem 9.2]). On the other hand

$$
d \zeta_{i}=\sum_{j=1}^{n}\left(X_{j}^{\prime} \zeta_{i}\right) \omega_{j}
$$

and therefore

$$
\alpha \omega=\left\{\sum_{j=1}^{n}(-1)^{j-1} X_{j}^{\prime} \zeta_{j}\right\} \omega
$$

This shows that

$$
\alpha=\sum_{j=1}^{n}(-1)^{j-1} X_{j}^{\prime} \zeta_{j}
$$

Hence in order to prove that $I(\alpha)=0$ implies $\sigma(\alpha)=0 .\left(\alpha \in C_{c}^{\infty}(G)\right)$, it is sufficient to verify that $\sigma\left(X^{\prime} \alpha\right)=0$ for every $X \in g_{0}$ and $\alpha \in C_{c}^{\infty}(G)$. Put

$$
\alpha_{t}(x)=\alpha(\exp (-t X) x) \quad(x \in G, t \in R) .
$$

Then if $b \in \mathfrak{B}$,

$$
\alpha_{t}(x, b)=\alpha(\exp (-t X) x, b)
$$

and from this it follows that if $\alpha^{\prime}=X^{\prime} \alpha, t^{-1}\left\{\alpha_{t}(x, b)-\alpha(x, b)\right\}$ converges uniformly on $G$ to $\alpha^{\prime}(x, b)$ as $t$ tends to zero. This implies that

$$
\sigma\left(\alpha^{\prime}\right)=\operatorname{Lim}_{t \rightarrow 0} \frac{1}{t}\left(\sigma\left(\alpha_{t}\right)-\sigma(\alpha)\right)=0
$$

since $\alpha_{t}=\lambda(\exp t X) \alpha$. Hence $\sigma\left(X^{\prime} \alpha\right)=0$ and our assertion is proved.

Select a function $\alpha_{0} \in C_{c}^{\infty}(G)$ such that $I\left(\alpha_{0}\right)=1$. Then if $\alpha \in C_{c}^{\infty}(G)$ and $\alpha^{\prime}=\alpha-I(\alpha) \alpha_{0}, I\left(\alpha^{\prime}\right)=0$. Hence $\sigma\left(\alpha^{\prime}\right)=0$ and therefore $\sigma(\alpha)=\sigma\left(\alpha_{0}\right) I(\alpha)$. This proves the lemma.

Lemma 37. The distribution $\tau_{\star}$ on ' $M$ is an invariant distribution.

It is obvious that if $\alpha \in C_{c}^{\infty}(G), \beta \in C_{c}^{\infty}\left({ }^{\prime} M\right)$ and $m \in M$,

$$
S_{\pi}\left(\alpha \times \beta^{m}\right)=S_{\pi}\left(\alpha^{\prime} \times \beta\right)
$$

where $\alpha^{\prime}(x)=\alpha\left(x m^{-1}\right)(x \in G)$. Hence if we choose $\alpha$ such that $\int_{G} \alpha(x) d x=1$,

$$
\tau_{\pi}\left(\beta^{m}\right)=S_{\pi}\left(\alpha \times \beta^{m}\right)=S_{\pi}\left(\alpha^{\prime} \times \beta\right)=\tau_{\pi}(\beta)
$$

and this shows that $\tau_{\pi}$ is invariant. 
Define the differential operator $\delta(z)(z \in \mathbb{Z})$ on ' $M$ as in Theorem 1.

LEMMA 38. $\delta(z) \tau_{\pi}=\chi_{\pi}(z) \tau_{\pi}(z \in 3)$ where $\chi_{\pi}$ is the infinitesimal character of $\pi$.

We use the notation of the proof of Lemma 34. Let $E_{\triangleright}$ denote the canonical projection [6(c), p. 225] of $\mathfrak{S}$ on $\mathfrak{S}_{\mathfrak{D}}\left(\mathfrak{D} \in \Omega_{\pi}\right)$. Then if $\phi_{\mathfrak{D}}(x)=\operatorname{sp}\left(E_{\mathfrak{D} \pi}(x) E_{\mathfrak{D}}\right)$ $(x \in G)$ it is obvious from Lemma 34 that

$$
Q(\alpha, \beta)=\int \alpha(x) \beta(m) \pi\left(x m x^{-1}\right) d x d m \quad\left(\alpha \in C_{c}^{\infty}(G), \beta \in C_{c}^{\infty}\left({ }^{\prime} M\right)\right)
$$

is of the trace class and therefore (see [6(d), §2])

$$
\operatorname{sp} Q(\alpha, \beta)=\sum_{\mathfrak{D} \in \Omega_{x}} \int \alpha(z) \beta(m) \phi_{\mathfrak{D}}\left(x m x^{-1}\right) d x d m .
$$

Also we know (see Lemmas 32 and 34 of [6(c) ]) that $\phi_{\circledast}$ is an analytic function on $G$ and

$$
\phi_{\mathfrak{D}}(x, z)=\chi_{\pi}(z) \phi_{\mathfrak{D}}(x) \quad(x \in G, z \in \mathbb{3}) .
$$

Put $\Phi_{\mathfrak{D}}(x ; m)=\phi_{\mathfrak{D}}\left(x m x^{-1}\right)\left(x \in G, m \in \in^{\prime} M\right)$. Then if $b \in \mathfrak{B}$ and $\nu \in \mathbb{M}$, we have seen in $\$ 5$ that

$$
\Phi_{\mathcal{D}}(x, b ; m, \nu)=\phi_{\mathfrak{D}}\left(x m x^{-1}, \operatorname{Ad}(x)\left(\Gamma_{m}(b \times \nu)\right)\right) .
$$

On the other hand if $z$ is a fixed element in 3 , we can, from Lemma 16, select $b_{1}, \cdots, b_{r} \in \mathbb{Q}^{\prime}, \nu_{1}, \cdots, \nu_{r} \in \mathfrak{M}$ and analytic functions $a_{1}, \cdots, a_{r}$ on ' $M$ such that

$$
\sum_{i=1}^{r} a_{i}(m) \Gamma_{m}\left(b_{i} \times \nu_{i}\right)+\Gamma_{m}\left(1 \times \delta_{m}(z)\right)=z .
$$

Therefore since $z^{x}=z$,

$$
\sum_{i=1}^{r} a_{i}(m) \Phi_{\mathfrak{D}}\left(x, b_{i} ; m, \nu_{i}\right)+\Phi_{\mathfrak{D}}\left(x ; m, \delta_{m}(z)\right)=\phi_{\mathfrak{D}}\left(x m x^{-1}, z\right)=\chi_{\pi}(z) \phi_{\mathfrak{D}}\left(x m x^{-1}\right) .
$$

Let $D_{i}$ and $\delta^{*}(z)$ respectively denote the adjoints of the differential operators $a_{i}(m) \nu_{i}$ and $\delta(z)$ on ' $M$. If $b \rightarrow b^{*}(b \in \mathfrak{B})$ is the anti-automorphism of $\mathfrak{B}$ which maps $X$ on $-X(X \in \mathfrak{g})$, it is obvious that $b_{i}^{*}$ is the adjoint of the differential operator $b_{i}$ on $G$. Hence

$$
\begin{aligned}
\chi_{\pi}(z) & \int \alpha(x) \beta(m) \phi_{\mathfrak{D}}\left(x m x^{-1}\right) d x d m \\
\quad= & \sum_{i=1}^{r} \int \alpha\left(x, b_{i}^{*}\right) \beta\left(m, D_{i}\right) \Phi_{\mathfrak{D}}(x ; m) d x d m+\int \alpha(x) \beta\left(m, \delta^{*}(z)\right) \Phi_{\mathfrak{D}}(x ; m) d x d m
\end{aligned}
$$


and therefore it follows from Lemma 34 that

$$
\chi_{\pi}(z) \operatorname{sp} Q(\alpha, \beta)=\sum_{i=1}^{r} \operatorname{sp} Q\left(b_{i}^{*} \alpha, D_{i} \beta\right)+\operatorname{sp} Q\left(\alpha, \delta^{*}(z) \beta\right) .
$$

Now assume that $I(\alpha)=1$ and apply Lemma 35. Then we get

$$
\chi_{\pi}(z) \tau_{\pi}(\beta)=\sum_{i=1}^{r} I\left(b_{i}^{*} \alpha\right) \tau_{\pi}\left(D_{i} \beta\right)+\tau_{\pi}\left(\delta^{*}(z) \beta\right) .
$$

But since $b_{i} \in \mathfrak{Q}^{\prime}$ the same holds for $b_{i}{ }^{*}$. On the other hand if $X \in \mathfrak{g}_{0}$ and $f \in C_{c}^{\infty}(G)$, it is obvious that $I\left(f_{t}\right)=I(f)$ where $f_{t}(x)=f(x \exp t X)(x \in G, t \in R)$. From this we conclude immediately that $I(X f)=0$. Hence $I\left(b_{i}{ }^{*} \alpha\right)=0(1 \leqq i \leqq r)$ and therefore

$$
\chi_{\pi}(z) \tau_{\pi}(\beta)=\tau_{\pi}\left(\delta^{*}(z) \beta\right) \quad\left(\beta \in C_{c}^{\infty}\left({ }^{\prime} M\right)\right) .
$$

This proves the statement of the lemma.

It now follows from Theorem 3 that $\tau_{\pi}$ is an analytic function on ' $M$. In order to prove Theorem 6 we still have to investigate the relationship between the two distributions $T_{\pi}$ and $\tau_{\pi}$. Put $q_{0}=q \cap g_{0}$. Then $g_{0}$ is the direct sum of $\mathfrak{q}_{0}$ and $\mathfrak{m}_{0}=\mathfrak{m} \cap \mathfrak{g}_{0}$. Let $U^{\prime}$ be an open neighborhood of 1 in $G$ such that if $U^{\prime \prime}=\left(U^{\prime}\right)^{-1} U^{\prime}$ then $U^{\prime \prime} \cap(\tilde{A} M) \subset M$ where $\widetilde{A}$ is the normalizer of $A$ in $G$. This is possible since $\tilde{A} M / M$ is a finite group (see Lemma 10). Let $G^{*}=G / M$ be the factor space consisting of all cosets of the form $x M(x \in G)$ and let $\sigma$ denote the natural mapping $x \rightarrow x^{*}$ of $G$ on $G^{*}$. We can select an open neighborhood $U^{*}$ of $1^{*}$ in $G^{*}$ and a regular analytic mapping $\phi$ of $U^{*}$ into $U^{\prime}$ such that $\left(\phi\left(x^{*}\right)\right)^{*}=x^{*}$ and $\phi\left(1^{*}\right)=1\left(x^{*} \in U^{*}\right)$. Let $d x^{*}$ denote the invariant measure on $G^{*}$. Define an analytic mapping $\psi$ of $U^{*} \times{ }^{\prime} M$ into $G$ as follows: $\psi\left(x^{*}, m\right)=\phi\left(x^{*}\right) m\left(\phi\left(x^{*}\right)\right)^{-1}\left(x^{*} \in U^{*}, m \in{ }^{\prime} M\right)$. Suppose $\psi\left(x_{1}^{*}, m_{1}\right)=\psi\left(x_{2}{ }^{*}, m_{2}\right)$ $\left(x_{1}^{*}, x_{2}^{*} \in U^{*}, m_{1}, m_{2} \in M\right)$. Then from Lemma 10

$$
\left(\phi\left(x_{1}^{*}\right)\right)^{-1} \phi\left(x_{2}^{*}\right) \in U^{\prime \prime} \cap(\tilde{A} M) \subset M
$$

and therefore $x_{2}{ }^{*}=x_{1}{ }^{*}, m_{2}=m_{1}$. This shows that $\psi$ is univalent on $U^{*} \times{ }^{\prime} M$. For $Y \in g_{0}$ and $x^{*} \in U^{*}$ put $\left({ }^{12}\right) Y_{x^{*}}^{*}=d \sigma_{x} Y$ where $x=\phi\left(x^{*}\right)$. Then if we identify the tangent space of $U^{*} \times{ }^{\prime} M$ at $\left(x^{*}, m\right)$ with $g_{0}$ under the linear isomorphism

$$
Y+X \rightarrow Y_{x^{*}}^{*} \times X \quad\left(Y \in \mathfrak{q}_{0}, X \in \mathfrak{m}_{0}\right),
$$

a simple calculation shows that

$$
d \psi_{x^{*}, m}(X+Y)=\left(\operatorname{Ad} \phi\left(x^{*}\right)\right) X+\operatorname{Ad} \phi\left(x^{*}\right)\left\{\operatorname{Ad}\left(m^{-1}\right)-I\right\}\left(d \phi_{x^{*}} Y_{x^{*}}^{*}\right) .
$$

(Here $I$ is the identity mapping of g.) Moreover $\sigma \circ \phi$ being the identity mapping on $U^{*}$,

(12) We follow here the terminulogy of Chevalley [2]. 


$$
d \sigma_{x}\left(d \phi_{x^{*}} Y_{x^{*}}^{*}-Y\right)=0
$$

if $x=\phi\left(x^{*}\right)$ and therefore

$$
d \phi_{x^{*}} Y_{x^{*}}^{*} \equiv Y \bmod \mathfrak{M}_{0}
$$

Therefore if $D_{x^{*}, m}=\left(\operatorname{Ad} \phi\left(x^{*}\right)\right)^{-1} d \psi_{x^{*}, m}$,

$$
\begin{aligned}
& D_{x^{*}, m} X=X \\
& D_{x^{*}, m} Y \equiv \operatorname{Ad}\left(m^{-1}\right) Y-Y \bmod \mathfrak{M}_{0}
\end{aligned}
$$$$
\left(X \in \mathfrak{m}_{0}\right) \text {, }
$$

and so it follows that

$$
\operatorname{det}\left(d \psi_{x^{*}, m}\right)=\operatorname{det} D_{x^{*}, m}=d_{+}(m) \neq 0 .
$$

This proves that $\psi$ is everywhere regular on $U^{*} \times{ }^{\prime} M$. Hence it maps $U^{*} \times{ }^{\prime} M$ topologically on an openneighborhood $N$ of $' M$ in $G$. We regard $N$ as an open submanifold of $G$. Let $f \in C_{c}^{\infty}(N)$ and put

$$
F\left(x^{*}, m\right)=f\left(\psi\left(x^{*}, m\right)\right) \quad\left(x^{*} \in U^{*}, m \in \mathcal{\prime}^{\prime} M\right) .
$$

Since $\psi$ defines an isomorphism of the two analytic manifolds $U^{*} \times{ }^{\prime} M$ and $N$, it is clear that $F \in C_{c}^{\infty}\left(U^{*} \times{ }^{\prime} M\right)$. Choose bases $\left(X_{1}, \cdots, X_{p}\right)$ and $\left(Y_{1}, \cdots, Y_{q}\right)$ for $\mathfrak{m}_{0}$ and $\mathfrak{q}_{0}$ respectively over $R$ and let $\omega^{*}$ and $\zeta$ denote the differential forms (of degrees $q$ and $p$ ) on $G^{*}$ and $M$ respectively corresponding to the measures $d x^{*}$ and $d m$. Then we may assume that

$$
\omega_{x^{*}}^{*}\left(d \sigma_{x} Y_{1}, \cdots, d \sigma_{x} Y_{q}\right)=\zeta_{m}\left(X_{1}, \cdots, X_{p}\right)=1 \quad(x \in G, m \in M) .
$$

On the other hand if $\omega$ is the differential form on $G$ of degree $p+q$ corresponding to the measure $d x$, we may assume that $\omega_{x}\left(Y_{1}, \cdots, Y_{q}, X_{1}, \cdots, X_{p}\right)=1$ $(x \in G)$. Now consider the form $\left(^{12}\right) \omega^{\prime}=(\delta \psi) \omega$ on $U^{*} \times{ }^{\prime} M$. Then it follows from the above result that

$$
\begin{aligned}
\omega_{x^{*}, m}^{\prime}\left(Y_{1}, \cdots, Y_{q}, X_{1}, \cdots X_{p}\right) \\
\quad=d_{+}(m) \omega_{y}\left(Y_{1}, \cdots, Y_{q}, X_{1} X_{1}, \cdots X_{p}\right)=d_{+}(m)
\end{aligned}
$$

where $y=\psi\left(x^{*}, m\right)\left(x^{*} \in U^{*}, m \in \in^{\prime} M\right)$ and this proves that

$$
\omega_{x^{*}, m}^{\prime}=d_{+}(m) \xi_{x^{*}, m}
$$

where $\xi$ is the direct product of $\omega^{*}$ and $\zeta$ on $U^{*} X^{\prime} M$ so that

$$
\begin{aligned}
\xi_{x^{*}, m}\left(Y_{1}, \cdots,\right. & \left.Y_{q}, X_{1}, \cdots, X_{p}\right) \\
& =\omega_{x^{*}}^{*}\left(\left(Y_{1}\right)_{x^{*}}^{*}, \cdots,\left(Y_{q}\right)_{x^{*}}^{*}\right) \zeta_{m}\left(X_{1}, \cdots, X_{p}\right)=1 .
\end{aligned}
$$

But this implies that

$$
\int_{N} f(x) d x=\int_{U^{*} X^{\prime} M} F\left(x^{*}, m\right)\left|d_{+}(m)\right| d x^{*} d m .
$$


Thus we have obtained the following result.

Lemma 39. If the measures $d x^{*}, d m$ and $d x$ on $G^{*}, M$ and $G$ respectively are suitably normalized,

$$
\int f(x) d x=\int_{U^{*} x^{*} M} f\left(\psi\left(x^{*}, m\right)\right)\left|d_{+}(m)\right| d x^{*} d m
$$

for every $f \in C_{c}^{\infty}(N)$.

Now $\psi$ being univalent and regular on $U^{*} \times{ }^{\prime} M$, there exists an analytic function $F_{\pi}$ on $N$ such that $F_{\pi}\left(\psi\left(x^{*}, m\right)\right)=\tau_{\pi}(m)\left(x^{*} \in U^{*}, m \in M\right)$.

LemMa 40. Suppose the various measures have been normalized in accordance with Lemma 39. Then

$$
T_{\pi}(f)=\int f(x) F_{\pi}(x) d x
$$

for every $f \in C_{c}^{\infty}(N)$.

For a given $f \in C_{c}^{\infty}(N)$ we can choose a compact subset $\omega^{*}$ of $U^{*}$ such that $f\left(\psi\left(x^{*}, m\right)\right)=0\left(x^{*} \in U^{*}, m \in \in^{\prime} M\right)$ unless $x^{*} \in \omega^{*}$. Let $\alpha^{*}$ be a function in $C_{c}^{\infty}\left(U^{*}\right)$ which is identically equal to 1 on $\omega^{*}$. The mapping $\left(x^{*}, m\right) \rightarrow \phi\left(x^{*}\right) m$ is easily seen to be regular and univalent on $U^{*} \times M$. Therefore it defines an analytic isomorphism of $U^{*} \times M$ with the open submanifold $U=\phi\left(U^{*}\right) M$ of $G$. Select $\beta \in C_{c}^{\infty}(M)$ such that $\int_{M} \beta(m) d m=1$. Then there exists an element $\alpha \in C_{c}^{\infty}(U)$ such that $\alpha\left(\phi\left(x^{*}\right) m\right)=\alpha^{*}\left(x^{*}\right) \beta(m)\left(x^{*} \in U^{*}, m \in M\right)$. Now $g$ being any indefinitely differentiable function on $N$, put $f^{\prime}=g f$. Then

$$
\begin{aligned}
\int_{\sigma_{\times} M} \alpha(x) f^{\prime}\left(x m x^{-1}\right)\left|d_{+}(m)\right| d x d m \\
=\int_{G^{*} \times M \times^{\cdot} M} \alpha^{*}\left(x^{*}\right) \beta\left(m_{1}\right) f^{\prime}\left(\psi\left(x^{*}, m_{1} m m_{1}^{-1}\right)\right)\left|d_{+}(m)\right| d x^{*} d m_{1} d m
\end{aligned}
$$

since $d x=d x^{*} d m_{1}$ if $x=\phi\left(x^{*}\right) m_{1}\left(x^{*} \in U^{*}, m_{1} \in M\right)$. But $d_{+}\left(m_{1}^{-1} m m_{1}\right)=d_{+}(m)$ and therefore

$$
\int_{\mathcal{M}_{M}} f^{\prime}\left(\psi\left(x^{*}, m_{1} m m_{1}^{-1}\right)\right)\left|d_{+}(m)\right| d m=\int_{\mathcal{M}_{M}} f^{\prime}\left(\psi\left(x^{*}, m\right)\right)\left|d_{+}(m)\right| d m .
$$

Moreover $\int_{M} \beta\left(m_{1}\right) d m_{1}=1$ and $\alpha^{*}=1$ on $\omega^{*}$. Hence

$$
\begin{aligned}
\int_{a_{X^{\prime} M}} \alpha(x) f^{\prime}\left(x m x^{-1}\right)\left|d_{+}(m)\right| d x d m & =\int_{G^{*} \times^{\prime} M} f^{\prime}\left(\psi\left(x^{*}, m\right)\right)\left|d_{+}(m)\right| d x^{*} d m \\
& =\int_{G} f^{\prime}(x) d x
\end{aligned}
$$


from Lemma 39. This proves that

$$
\int f(x) g(x) d x=\int_{G \times^{\circ} M} \alpha(x) f\left(x m x^{-1}\right) g\left(x m x^{-1}\right)\left|d_{+}(m)\right| d x d m
$$

if $g \in C^{\infty}(N)$. On the other hand

$$
T_{\boldsymbol{\pi}}(f)=\sum_{\mathfrak{D} \in \Omega_{\pi}} \int f(x) \phi_{\mathfrak{D}}(x) d x
$$

in the notation of the proof of Lemma 38. Therefore

$$
T_{\pi}(f)=\sum_{\mathfrak{D} \in \Omega_{\pi}} \int \alpha(x) f\left(x m x^{-1}\right) \phi_{\mathfrak{D}}\left(x m x^{-1}\right)\left|d_{+}(m)\right| d x d m .
$$

Now put

$$
f_{1}(x, m)=\alpha(x) f\left(x m x^{-1}\right)\left|d_{+}(m)\right| \quad\left(x \in G, m \in{ }^{\prime} M\right) .
$$

Then $f_{1} \in C_{c}^{\infty}\left(G \times{ }^{\prime} M\right)$ and it follows from Lemma 34 that the right hand side of the above equation is equal to $S_{\pi}\left(f_{1}\right)$. On the other hand

$$
S_{\pi}\left(f_{1}\right)=\int_{G X^{\prime} M} f_{1}(x, m) \tau_{\pi}(m) d x d m
$$

from Lemma 35 (see Schwartz [11, p. 108]). Therefore

$$
T_{\pi}(f)=\int_{G \times{ }^{\prime} M} \alpha(x) f\left(x m x^{-1}\right) \tau_{\pi}(m)\left|d_{+}(m)\right| d x d m=\int_{G} f(x) F_{\pi}(x) d x
$$

from our result above. This proves Lemma 40 and since $N \supset^{\prime} M \supset^{\prime} A$, Theorem 6 follows.

For any quasi-simple representation $\pi$ of $G$ define $\eta_{\pi}, \chi_{\pi}$ and $T_{\pi}$ as above and let $F_{x}$ be the analytic function (on ' $G$ ) which coincides with $T_{\pi}$ on ' $G$. From the corollary to Lemma 12 we can choose a finite number of connected components $G_{1}, \cdots, G_{N}$ of ' $G$ such that ' $G=\cup_{i=1}^{N} Z G_{i}$. Then if $w$ is the order of the Weyl group of $\mathfrak{g}$ (with respect to any Cartan subalgebra $\mathfrak{h}$ ), we obtain the following result by combining Theorem 4 and Lemma 40.

Theorem 7. Suppose $\eta$ and $\chi$ respectively are given homomorphisms of $Z$ and 3 into $C$ and $\omega$ is a collection of quasi-simple representations $\pi$ of $G$ such that $\eta=\eta_{\pi}$ and $\chi_{=}=\chi_{\pi}$. Then the dimension of the linear space spanned over $C$ by the functions $F_{\pi}(\pi \in \omega)$ cannot exceed $N w$.

Since

$$
\int f\left(z^{-1} x\right) \pi(x) d x=\int f(x) \pi(z x) d x=\eta(z) \int f(x) \pi(x) d x \quad(z \in Z)
$$

and

$$
T_{\pi}(f)=T_{\pi}\left(f^{y}\right)
$$


for $f \in C_{c}^{\infty}(G)$ and $\pi \in \omega$, it is obvious that $F_{\pi}(z x)=\eta(z) F_{\pi}(x)$ and $F_{\pi}\left(y x y^{-1}\right)$ $=F_{\pi}(x)\left(x \in \in^{\prime} G\right)$. Therefore if $V$ is the linear space spanned by $F_{\pi}(\pi \in \omega)$, similar relations hold for every $F \in V$. Let $F_{i}$ denote the restriction on $G_{i}$ of a function $F$ in $V$. If $F$ vanishes identically on some nonempty open subset of $G_{i}$ then $F_{i}=0$. Now the set $\bigcup_{x \in G} x G_{i} x^{-1}$ is connected since it is the image of $G \times G_{i}$ under the continuous mapping $(x, y) \rightarrow x y x^{-1}$. Hence it follows that $x G_{i} x^{-1}=G_{i}(x \in G)$. Therefore from Lemma $11, G_{i}$ must meet at least one Cartan subgroup $A_{i}$ of $G$ for which $\theta\left(A_{i}\right)=A_{i}$. Let $A_{i}^{\prime}$ be the set of regular elements in $A_{i}$. By Lemma 8 we can select a nonempty open connected subset $B_{i}$ of $A_{i}^{\prime}$ such that $B_{i} \subset A_{i} \cap G_{i}$. One proves without difficulty that $U_{i}=U_{x \in G} x B_{i} x^{-1}$ is open in $G$ and therefore if $F$ is everywhere zero on $B_{i}$, $F_{i}=0$. Let $\bar{F}_{i}$ denote the restriction of $F$ on $B_{i}$ and let $\bar{V}_{i}$ be the linear space consisting of all $\bar{F}_{i}(F \in V)$. It follows from Lemma 38 and Theorem 4 that $\operatorname{dim} \bar{V}_{i} \leqq w$. Now $\bar{V}$ being the direct sum of $\bar{V}_{i}(1 \leqq i \leqq N)$, consider the linear mapping $F \rightarrow \bar{F}=\left(\bar{F}_{1}, \cdots, \bar{F}_{N}\right)$ of $V$ into $\bar{V}$. Since ${ }^{\prime} G=\bigcup_{i=1}^{N} Z G_{i}$, it is clear that $F=0$ whenever $\bar{F}=0$. Hence $\operatorname{dim} V \leqq \operatorname{dim} \bar{V} \leqq N w$.

In view of Theorem 7 we can choose a finite number of representations $\pi_{1}, \cdots, \pi_{r}$ in $\omega$ such that $F_{\pi_{1}}, \cdots, F_{\pi_{r}}$ form a base for $V$. Then for any $\pi \in \omega, F_{\pi}=c_{1} F_{\pi_{1}}+\cdots+c_{r} F_{\pi_{r}}$ where $c_{1}, \cdots, c_{r}$ are complex numbers. From this one would like to deduce that $T_{x}=c_{1} T_{x_{1}}+\cdots+c_{r} T_{\pi_{r}}$. Although this conclusion seems to be in agreement with experience, I have not succeeded in constructing a proof of it. If true it has certain interesting consequences (see $[6(h)])$.

12. Closer study of a special case. We now make the two assumptions of $\$ 10$ on $G$ and use the notation introduced there. Put

$$
\Delta_{-}^{\prime}=\prod_{\alpha \in P_{-}}\left(1-\xi_{\alpha}^{-1}\right)
$$

and normalize the Haar measures $d k$ and $d h$ on $K$ and $A$ respectively in such a way that

$$
\int_{K} d k=\int_{A}\left|\Delta_{-}^{\prime}(h)\right|^{2} d h=1 .
$$

Leмma 41. Let $\pi$ be a quasi-simple representation of $G$. Then for any $f \in C^{\infty}(K)$ the operator

$$
\int_{K} f(k) \pi(k) d k
$$

is summable and if $\tau_{\pi}(f)$ denotes its trace, the mapping

$$
\tau_{\pi}: f \rightarrow \tau_{\pi}(f)
$$

is a distribution on $K$. 
This follows immediately if we apply the reasoning of $[6(d), \S 5]$ to the representation $k \rightarrow \pi(k)$ of $K$.

COROLLARY. The distribution $\tau_{\pi}$ defined above coincides on ' $K$ with that of Lemma 35.

For suppose $\alpha \in C_{c}^{\infty}(G)$ and $f \in C^{\infty}(K)$. Since $\int_{K} f(k) \pi(k) d k$ is summable, it follows without difficulty (see $[6(d)$, p. 237]) that

$$
\int_{G \times K} \alpha(x) f(k) \pi\left(x k x^{-1}\right) d x d k
$$

is of the trace class and

$$
\begin{aligned}
\operatorname{sp}\left(\int_{G \times K} \alpha(x) f(k) \pi\left(x k x^{-1}\right) d x d k\right) & =\int_{G} \alpha(x) d x \operatorname{sp}\left(\int_{K} f(k) \pi(k) d k\right) \\
& =\int_{G} \alpha(x) d x \tau_{\pi}(f) .
\end{aligned}
$$

Our assertion is a direct consequence of this fact.

On the other hand we have the following two lemmas.

Lemma 42. For any $f \in C^{\infty}(A)$ the operator

$$
\int_{A \times K} f(h) \Delta_{-}^{\prime}(h) \pi\left(k h k^{-1}\right) d h d k
$$

is summable and if $\sigma_{\pi}(f)$ denotes its trace, the mapping

$$
\sigma_{\pi}: f \rightarrow \sigma_{\pi}(f)
$$

is a distribution on $A$.

The proof of this is substantially the same as that of Lemma 24 of $[6(\mathrm{~g})]$.

Now put $\rho_{-}=\sum_{\alpha \in P_{-}} \alpha$. Then if $s \in W_{-}, s \rho_{-}-\rho_{-}$is a linear combination of roots in $P_{-}$with integer coefficients (see Weyl [14(b)]). Hence $\xi_{s}^{-}=\xi_{\Delta \rho_{-}-\rho_{-}}$ is a well-defined character of $A$ and

$$
\Delta_{-}^{\prime}=\sum_{, \in W_{-}} \epsilon(s) \xi_{s}^{-} .
$$

Now if we apply the method of proof of Lemma 29 to the pair $(K, A)$ we obtain the following result.

LEMmA 43. For any $f \in C^{\infty}(A)$ there exists a unique function $\phi_{f} \in I^{\infty}(K)$ such that

$$
\Delta_{-}^{\prime}(h) \phi_{f}(h)=\sum_{\imath \in W_{-}} \epsilon(s) \bar{\xi}_{e}^{-}(h) f^{\bullet}(h) \quad(h \in A) .
$$


COROLlaRY. $\sigma_{\pi}$ coincides on 'A with the analytic function $h \rightarrow \Delta_{-}^{\prime}(h) \tau_{\pi}(h)$ $\left(h \in \mathcal{\prime}^{\prime} A\right)$.

For suppose $f \in C_{c}^{\infty}(' A)$. Then it is obvious that $\phi_{f} \in I_{c}^{\infty}\left({ }^{\prime} K\right)$ and

$$
\int_{K} \phi_{f}(k) \pi(k) d k=\int_{A \times K} \phi_{f}(h)\left|\Delta_{-}^{\prime}(h)\right|{ }^{2} \pi\left(k h k^{-1}\right) d h d k .
$$

On the other hand if $h \in A$ and $s \in W_{-}$,

$$
\int_{K} \pi\left(k h^{8} k^{-1}\right) d k=\int_{K} \pi\left(k h k^{-1}\right) d k
$$

and

$$
\Delta_{-}^{\prime}\left(h^{0}\right)=\epsilon(s) \xi_{\varepsilon}^{-}\left(h^{0}\right) \Delta_{-}^{\prime}(h)
$$

Therefore $\left({ }^{13}\right)$

$$
\int_{K} \phi_{f}(k) \pi(k) d k=w_{-} \int_{A \times K} f(h)\left(\operatorname{conj} \Delta_{-}^{\prime}(h)\right) \pi\left(k h k^{-1}\right) d h d k
$$

where $w_{-}$is the order of $W_{-}$. On the other hand since $\tau_{\pi}$ coincides with an analytic function on ' $K$,

$$
\operatorname{sp}\left(\int_{K} \phi_{f}(k) \pi(k) d k\right)=\int_{K} \phi_{f}(k) \tau_{\pi}(k) d k=\int_{A} \phi_{f}(h)\left|\Delta_{-}^{\prime}(h)\right|{ }^{2} \tau_{\pi}(h) d h .
$$

Again since $\tau_{\pi}$ is an invariant function on ' $K$ (Lemma 37), $\tau_{\pi}\left(h^{\star}\right)=\tau_{\pi}(h)$ $\left(s \in W_{-}, h \in \in^{\prime} A\right)$ and therefore

$$
\int_{A} \phi_{f}(h)\left|\Delta_{-}^{\prime}(h)\right|{ }^{2} \tau_{\pi}(h) d h=w_{-} \int_{A} f(h) \operatorname{conj} \Delta_{-}^{\prime}(h) \tau_{\pi}(h) d h .
$$

But

$$
\operatorname{conj} \Delta_{-}^{\prime}=\prod_{\alpha \in P_{-}}\left(1-\xi_{\alpha}\right)=(-1)^{r} \xi_{2 \rho-} \Delta_{-}^{\prime}
$$

where $r$ is the number of roots in $P_{-}$. Hence replacing $f$ by $f \xi_{2 \rho_{-}}^{-1}$, we obtain

$$
\operatorname{sp}\left(\int_{A \times \boldsymbol{K}} f(h) \Delta_{-}^{\prime}(h) \pi\left(k h k^{-1}\right) d h d k\right)=\int f(h)\left(\Delta_{-}^{\prime}(h) \tau_{\pi}(h) d h .\right.
$$

This proves our assertion.

We shall now try to obtain some information regarding the possible "singularities" of $\sigma_{\pi}$ on $A$. In an earlier paper $[6(g), \S 10]$ we made a detailed study of certain examples and found that there one could always select a

${ }^{(13)} \operatorname{conj} c$ denotes the conjugate of a complex number $c$. 
meromorphic function on $A$ so as to coincide with $\sigma_{\pi}$ on ' $A$. The following result, although it is weaker, holds under quite general conditions. Define $\Delta^{\prime}$ as in $\$ 10$.

TheOREM 8. Let $S_{\pi}$ be the distribution on $A$ given by

$$
S_{\pi}(f)=\operatorname{sp}\left\{\int_{A \times K}\left|\Delta^{\prime}(h)\right|^{2} \sum_{: \in W} f^{*}(h) \pi\left(k h k^{-1}\right) d h d k\right\} \quad\left(f \in C^{\infty}(A)\right) .
$$

Then $S_{\pi}$ coincides with an analytic function on $A$.

That the above operator is actually summable and $S_{\pi}$ is a distribution follows immediately from Lemma 42. Moreover we have seen that if $g \in C^{\infty}(K)$ and $\alpha \in C_{c}^{\infty}(G)$,

$$
\int \alpha(x) g(k) \pi\left(x k x^{-1}\right) d x d k
$$

is of the trace class and

$$
\operatorname{sp}\left(\int_{G \times K} \alpha(x) g(k) \pi\left(x k x^{-1}\right) d x d k\right)=\int_{G} \alpha(x) d x \tau_{\pi}(g) .
$$

Hence we conclude that

$$
\int_{G} \alpha(x) d x \tau_{\pi}(g)=\sum_{D \in \Omega_{\pi}} \int_{G \times K} \alpha(x) g(k) \phi_{\mathfrak{D}}\left(x k x^{-1}\right) d x d k
$$

the series being absolutely convergent. (Here the notation is the same as in the proof of Lemma 38.) Now choose $\alpha$ such that $\int \alpha(x) d x=1$. Then

$$
\tau_{\pi}\left(d_{+} g\right)=\sum_{D \in \Omega_{\pi}} \int_{G \times \mathbb{K}} \alpha(x) d_{+}(k) g(k) \phi_{D}\left(x k x^{-1}\right) d x d k
$$

for $g \in C^{\infty}(K)$. On the other hand $\omega$ being the Casimir operator of $\mathfrak{g}$,

$$
\phi_{\mathfrak{D}}(x, \omega)=\chi_{\pi}(\omega) \phi_{\mathfrak{D}}(x) \quad\left(x \in G, \mathfrak{D} \in \Omega_{\pi}\right)
$$

as we saw in $\$ 11$. Therefore

$$
\chi_{\pi}(\omega) \tau_{\pi}\left(d_{+} g\right)=\sum_{D \in \Omega_{\pi}} \int_{G \times K} \alpha(x) d_{+}(k) g(k) \phi_{D}\left(x k x^{-1}, \omega\right) d x d k .
$$

Now put

$$
\Phi_{\mathfrak{D}}(x ; k)=\phi_{\mathfrak{D}}\left(x k x^{-1}\right) \quad\left(x \in G, k \in K, \mathfrak{D} \in \Omega_{\pi}\right)
$$

and define $\gamma_{\pi}(k)$ and $D$ as in Lemmas 31 and 32. We have seen during the proof of Lemma 31 that 


$$
\gamma_{\omega}(k)=\sum_{i=1}^{r} a_{i}(k)\left(b_{i} \times 1\right)+1 \times D_{k}
$$

where $b_{1}, \cdots, b_{r}$ are homogeneous elements in $\mathfrak{Q}$ of degree $2, a_{1}, \cdots, a_{r}$ are analytic functions on $K$ and $D_{k}$ is the local expression at $k$ of the differential operator $D$. Since $\omega^{x}=\omega(x \in G)$ and $\Gamma_{k}\left(\gamma_{\omega}(k)\right)=d_{+}(k) \omega(k \in K)$, we conclude (see \$5) that

$$
\sum_{i=1}^{r} a_{i}(k) \Phi_{\mathfrak{D}}\left(x, b_{i} ; k\right)+\Phi_{\mathfrak{D}}\left(x ; k, D_{k}\right)=d_{+}(k) \phi_{\mathfrak{D}}\left(x k x^{-1}, \omega\right)
$$

and therefore

$$
\begin{aligned}
& \int_{G \times K} \alpha(x) d_{+}(k) g(k) \phi_{\mathfrak{D}}\left(x k x^{-1}, \omega\right) d x d k \\
& =\sum_{i=1}^{r} \int_{G \times K} \alpha\left(x, b_{i}^{*}\right) a_{i}(k) g(k) \phi_{\mathfrak{D}}\left(x k x^{-1}\right) d x d k+\int_{G \times K} \alpha(x) g\left(k, D^{*}\right) \phi_{\mathfrak{D}}\left(x k x^{-1}\right) d x d k
\end{aligned}
$$

where $D^{*}$ is the adjoint of $D$ and $b_{i}{ }^{*}$ is the image of $b_{i}$ under the anti-automorphism of $\mathfrak{B}$ which maps $X$ on $-X(X \in \mathfrak{g})$. Summing over all $\mathfrak{D} \in \Omega_{\pi}$, we get

$$
\chi_{\star}(\omega) \tau_{\pi}\left(d_{+} g\right)=\sum_{i=1}^{r} \int_{G} \alpha\left(x, b_{i}^{*}\right) d x \tau_{\pi}\left(a_{i} g\right)+\tau_{\pi}\left(D^{*} g\right) .
$$

But $b_{i}^{*}$ being homogeneous of degree 2 in $\mathfrak{B}$, it is obvious that

$$
\int_{G} \alpha\left(x, b_{i}^{*}\right) d x=0
$$

and therefore

$$
\tau_{\pi}\left(D^{*} g\right)=\chi_{\pi}(\omega) \tau_{\pi}\left(d_{+} g\right) \quad\left(g \in C^{\infty}(K)\right) .
$$

This proves that $D \tau_{\pi}=\chi_{\pi}(\omega) d_{+} \tau_{\pi}$.

Now define the mapping $f \rightarrow F_{f}$ of $C^{\infty}(A)$ into $I^{\infty}(K)$ as in Corollary 2 to Lemma 29 and let $\Phi$ denote the distribution $f \rightarrow \tau_{\pi}\left(d_{+} F_{f}\right)\left(f \in C^{\infty}(A)\right)$. Then it follows from Theorem 5 that $\Phi$ coincides with an analytic function on $A$. On the other hand if $f \in C^{\infty}(A)$,

$$
\int_{K} d_{+}(k) F_{f}(k) \pi(k) d k=\int_{A \times K} d_{+}(h)\left|\Delta_{-}^{\prime}(h)\right|{ }^{2} F_{f}(h) \pi\left(k h k^{-1}\right) d h d k
$$

and $d_{+}(h)\left|\Delta_{-}^{\prime}(h)\right|^{2}=\left|\Delta^{\prime}(h)\right|^{2}(h \in A)$. Now put

$$
g=\sum_{s \in W} f^{*}
$$

Then 


$$
\Delta^{\prime}(h) F_{o}(h)=\left(\sum_{s \in W} \epsilon(s) \xi_{s}(h)\right) g(h)=\Delta^{\prime}(h) g(h)
$$

and

$$
\begin{aligned}
\Phi(g) & =\tau_{\pi}\left(d_{+} F_{\vartheta}\right)=\operatorname{sp}\left(\int_{K} d_{+}(k) F_{\vartheta}(k) \pi(k) d k\right) \\
& =\operatorname{sp}\left(\int_{A \times K}\left|\Delta^{\prime}(h)\right|^{2} g(h) \pi\left(k h k^{-1}\right) d h d k\right) .
\end{aligned}
$$

Therefore if we put $\psi(h)=\sum_{s \in W} \Phi\left(h^{s}\right)(h \in A)$, it is clear that

$$
S_{\boldsymbol{x}}(f)=\int_{\boldsymbol{A}} f(h) \psi(h) d h
$$

This shows that $S_{\pi}$ coincides with $\psi$ on $A$.

The following lemma establishes a connection with finite-dimensional representations.

Lemma 44. Let $S_{\pi}$ be the analytic function on $A$ corresponding to Theorem 8. Then there exists an irreducible finite-dimensional representation $\sigma$ of $G$ and $a$ complex number $c$ such that

$$
S_{\pi}(h)=c\left|\Delta^{\prime}(h)\right|^{2} T_{\sigma}(h) \quad(h \in A) .
$$

Here $T_{\sigma}$ is the character of $\sigma$.

Let $A^{\prime}$ be the set of regular elements in $A$. It is clear that if $h \in A^{\prime}$, the same holds for $h^{s}(s \in W)$. It follows from the corollary to Lemma 43 that

$$
S_{\pi}(h)=\left|\Delta^{\prime}(h)\right|^{2} \bar{\tau}_{\pi}(h) \quad\left(h \in A^{\prime}\right)
$$

where

$$
\bar{\tau}_{\pi}(h)=\sum_{s \in W} \tau_{\pi}\left(h^{s}\right)
$$

Let $\mathfrak{h}_{0}^{\prime}$ be the complete inverse image of $A^{\prime}$ under the exponential mapping of $\mathfrak{h}_{0}$ into $A$ and let $V$ be a nonempty open connected subset of $\mathfrak{h}_{0}^{\prime}$. Then from Theorem 4,

$$
S_{\pi}(\exp H)=\Delta(H) \sum_{s \in W} p_{s}(H) e^{s \Delta(H)}
$$

where $\Lambda$ is a linear function and $p_{s}(s \in W)$ are polynomial functions on $\mathfrak{h}$. $(\Delta(H)$ is defined as in $\S 8$.) But since both sides of this equation may be regarded as analytic functions on $\mathfrak{h}_{0}$, they must be equal everywhere. Moreover we may assume that $p_{s t}=p_{s}$ if $t \Lambda=\Lambda(s, t \in W)$. Then since $S_{\pi}(\exp t H)$ $=S_{\pi}(\exp H)\left(t \in W, H \in \mathfrak{h}_{0}\right)$ we conclude from Lemma 41 of [6(b)] that 


$$
\epsilon(t) p_{s}(H)=p_{t s}(t H) \quad\left(s, t \in W, H \in \mathfrak{h}_{0}\right) .
$$

Hence if we put $p=p_{1}$ and define $p^{s}$ by $p^{s}(H)=p\left(s^{-1} H\right)(s \in W, H \in \mathfrak{h})$, it follows that $p_{s}=\epsilon(s) p^{s}$. Therefore

$$
S_{\pi}(\exp H)=\Delta(H) \sum_{s \in W} \epsilon(s) p^{s}(H) e^{s \Delta(H)} \quad\left(H \in \mathfrak{h}_{0}\right) .
$$

Let $H_{0}$ be an element in $\mathfrak{h}_{0}$ such that $\exp H_{0}=1$. Then $e^{\alpha\left(H_{0}\right)}=1$ for every root $\alpha$ and therefore $\Delta\left(H+H_{0}\right)=e^{-\rho\left(B_{0}\right)} \Delta(H) \quad(H \in \mathfrak{h})$. But since $S_{\pi}(\exp H)$ $=S_{\pi}\left(\exp \left(H+H_{0}\right)\right)$, we conclude that

$$
p^{s}\left(H+H_{0}\right) \exp \left(s \Lambda\left(H_{0}\right)-\rho\left(H_{0}\right)\right)=p^{s}(H) \quad\left(s \in W, H \in \mathfrak{h}_{0}\right) .
$$

In particular if $s=1$,

$$
p(H)=p\left(H+H_{0}\right) \exp \left(\Lambda\left(H_{0}\right)-\rho\left(H_{0}\right)\right) .
$$

Now we may suppose that $S_{\pi} \neq 0$ for otherwise the statement of the lemma is true trivially. Then $p \neq 0$ and if $p^{\prime}$ is the homogeneous component of $p$ of the highest degree, it is obvious that

$$
p^{\prime}=p^{\prime} \exp \left(\Lambda\left(H_{0}\right)-\rho\left(H_{0}\right)\right)
$$

so that $\exp \left(\Lambda\left(H_{0}\right)-\rho\left(H_{0}\right)\right)=1$ and $p(H)=p\left(H+H_{0}\right)$. This means that $p$ can be regarded as a continuous function on $A$ and so it must be bounded. But a polynomial function cannot be bounded on $\mathfrak{h}_{0}$ unless it is a constant. Moreover it is clear that there exists a character $\theta$ of $A$ such that

$$
\theta(\exp H)=\exp (\Lambda(H)-\rho(H)) \quad\left(H \in \mathfrak{h}_{0}\right) .
$$

Therefore

$$
S_{\mathbf{x}}(h)=c^{\prime}\left(\operatorname{conj} \Delta^{\prime}(h)\right) \sum_{s \in W} \epsilon(s) \xi_{s}(h) \theta^{s}(h)
$$

where $c^{\prime}$ is a constant. But as we have seen during the proof of Lemma 29, there exists an irreducible finite-dimensional representation $\sigma$ of $G$ such that

$$
\sum_{s \in W} \epsilon(s) \xi_{s}(h) \theta^{s}(h)=\epsilon \Delta^{\prime}(h) T_{\sigma}(h)
$$

where $T_{\sigma}$ is the character of $\sigma$ and $\epsilon= \pm 1$. Therefore if $c=\epsilon c^{\prime}$,

$$
S_{\pi}(h)=c\left|\Delta^{\prime}(h)\right|{ }^{2} T_{\sigma}(h) \quad(h \in A) .
$$

REFERENCES

1. E. Cartan, (a) Ann. Ecole Norm. vol. 44 (1927) pp. 345-467.

(b) J. Math. Pures Appl. vol. 8 (1929) pp. 1-33.

2. C. Chevalley, Theory of Lie groups, Princeton University Press, 1946.

3. L. Gårding, Math. Scand. vol. 1 (1953) pp. 55-72.

4. I. M. Gelfand and M. A. Naimark, Trudi Mat. Inst. Steklova vol. 36 (1950).

5. R. Godement, Trans. Amer. Math. Soc. vol. 73 (1953) pp. 496-556. 
6. Harish-Chandra, (a) Ann. of Math. vol. 50 (1949) pp. 900-915.

(b) Trans. Amer. Math. Soc. vol. 70 (1951) pp. 28-96.

(c) Trans. Amer. Math. Soc. vol. 75 (1953) pp. 185-243.

(d) Trans. Amer. Math. Soc. vol. 76 (1954) pp. 234-253.

(e) Trans. Amer. Math. Soc. vol. 76 (1954) pp. 485-528.

(f) Amer. J. Math. vol. 77 (1955) pp. 743-777.

(g) Amer. J. Math. vol. 78 (1956) pp. 1-41.

(h) Bull. Amer. Math. Soc. vol. 61 (1955) pp. 389-396.

7. K. Iwasawa, Ann. of Math. vol. 50 (1949) pp. 507-557.

8. F. John, Proceedings of the Symposium on Spectral Theory and Differential Problems, Stillwater, Okla., 1951, pp. 113-175.

9. J. L. Koszul, Bull. Soc. Math. France vol. 78 (1950) pp. 65-127.

10. G. D. Mostow, Bull. Amer. Math. Soc. vol. 55 (1949) pp. 969-980.

11. L. Schwartz, Theorie des distributions I, Paris, Hermann, 1950.

12. E. Stiefel, Comment. Math. Helv. vol. 14 (1942) pp. 350-380.

13. B. L. van der Waerden, Moderne Algebra, Berlin, Springer, 1937.

14. H. Weyl, (a) Math. Zeit. vol. 24 (1925) pp. 328-395.

(b) The structure and representations of continuous groups, Princeton, The Institute for Advanced Study, 1935.

(c) The classical groups, Princeton University Press, 1939.

InSTITUTE For AdVANCED STUdy,

Princeton, N. J.

Columbia UNiversity,

NEW YORK, N. Y. 